Linköping Studies in Science and Technology

Dissertations, No. 1672

\title{
Signal Reconstruction Algorithms for Time-Interleaved ADCs
}

Anu Kalidas Muralidharan Pillai

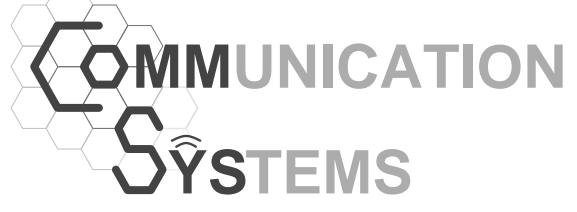

Division of Communication Systems

Department of Electrical Engineering (ISY)

Linköping University, SE-581 83 Linköping, Sweden

www.commsys.isy.liu.se

Linköping 2015 
Signal Reconstruction Algorithms for Time-Interleaved ADCs

(c) 2015 Anu Kalidas M. Pillai, unless otherwise noted.

ISBN 978-91-7519-062-4

ISSN 0345-7524

Printed in Sweden by LiU-Tryck, Linköping 2015 


\section{Abstract}

An analog-to-digital converter (ADC) is a key component in many electronic systems. It is used to convert analog signals to the equivalent digital form. The conversion involves sampling which is the process of converting a continuous-time signal to a sequence of discrete-time samples, and quantization in which each sampled value is represented using a finite number of bits. The sampling rate and the effective resolution (number of bits) are two key ADC performance metrics. Today, ADCs form a major bottleneck in many applications like communication systems since it is difficult to simultaneously achieve high sampling rate and high resolution. Among the various $\mathrm{ADC}$ architectures, the time-interleaved analog-to-digital converter (TI-ADC) has emerged as a popular choice for achieving very high sampling rates and resolutions. At the principle level, by interleaving the outputs of $M$ identical channel ADCs, a TI-ADC could achieve the same resolution as that of a channel ADC but with $M$ times higher bandwidth. However, in practice, mismatches between the channel ADCs result in a nonuniformly sampled signal at the output of a TI-ADC which reduces the achievable resolution. Often, in TI-ADC implementations, digital reconstructors are used to recover the uniform-grid samples from the nonuniformly sampled signal at the output of the TI-ADC. Since such reconstructors operate at the TI-ADC output rate, reducing the number of computations required per corrected output sample helps to reduce the power consumed by the TI-ADC. Also, as the mismatch parameters change occasionally, the reconstructor should support online reconfiguration with minimal or no redesign. Further, it is advantageous to have reconstruction schemes that require fewer coefficient updates during reconfiguration. In this thesis, we focus on reducing the design and implementation complexities of nonrecursive finite-length impulse response (FIR) reconstructors. We propose efficient reconstruction schemes for three classes of nonuniformly sampled signals that can occur at the output of TI-ADCs. 
Firstly, we consider a class of nonuniformly sampled signals that occur as a result of static timing mismatch errors or due to channel mismatches in TI-ADCs. For this type of nonuniformly sampled signals, we propose three reconstructors which utilize a two-rate approach to derive the corresponding single-rate structure. The two-rate based reconstructors move part of the complexity to a symmetric filter and also simplifies the reconstruction problem. The complexity reduction stems from the fact that half of the impulse response coefficients of the symmetric filter are equal to zero and that, compared to the original reconstruction problem, the simplified problem requires only a simpler reconstructor.

Next, we consider the class of nonuniformly sampled signals that occur when a TI-ADC is used for sub-Nyquist cyclic nonuniform sampling (CNUS) of sparse multi-band signals. Sub-Nyquist sampling utilizes the sparsities in the analog signal to sample the signal at a lower rate. However, the reduced sampling rate comes at the cost of additional digital signal processing that is needed to reconstruct the uniform-grid sequence from the sub-Nyquist sampled sequence obtained via CNUS. The existing reconstruction scheme is computationally intensive and time consuming and offsets the gains obtained from the reduced sampling rate. Also, in applications where the band locations of the sparse multi-band signal can change from time to time, the reconstructor should support online reconfigurability. Here, we propose a reconstruction scheme that reduces the computational complexity of the reconstructor and at the same time, simplifies the online reconfigurability of the reconstructor.

Finally, we consider a class of nonuniformly sampled signals which occur at the output of TI-ADCs that use some of the input sampling instants for sampling a known calibration signal. The samples corresponding to the calibration signal are used for estimating the channel mismatch parameters. In such TI-ADCs, nonuniform sampling is due to the mismatches between the channel ADCs and due to the missing input samples corresponding to the sampling instants reserved for the calibration signal. We propose three reconstruction schemes for such nonuniformly sampled signals and show using design examples that, compared to a previous solution, the proposed schemes require substantially lower computational complexity. 


\section{Populärvetenskaplig Sammanfattning}

En analog-till-digital-omvandlare (A/D-omvandlare) är en elektronisk komponent som används för att omvandla information (signaler) från analog till digital form. A/D-omvandlare är både nyckelkomponenter och flaskhalsar i många sammanhang, $\mathrm{t}$ ex i kommunikationssystem som behöver hantera mycket information per tidsenhet. Ju mer information som behöver omvandlas, desto svårare är det att praktiskt konstruera en $\mathrm{A} / \mathrm{D}$-omvandlare som klarar av att utföra detta utan fel. Prestandan hos A/D-omvandlare mäts i form av datatakt (samplingstakt), vilket anger antal sampel (mätvärden) per sekund, och den effektiva upplösningen (antal bitar) vilken anger den numeriska precisionen hos varje sampel. Det är framför allt svårt att samtidigt erhålla hög datatakt och hög upplösning.

Ett sätt att öka datatakten är att använda en så kallade sammanflätad A/Domvandlare. I en sådan används två eller flera parallella omvandlare som tar hand om olika sampel. Om $M$ omvandlare används parallellt erhålles då en $M$-fald ökning av datatakten. Emellertid finns det alltid analoga matchningsfel mellan de parallella omvandlarna. Detta ger upphov till så kallad vikningsdistorsion vilket i sin tur degraderar upplösningen. För att den sammanflätade A/D-omvandlaren ska uppnå samma upplösning som de individuella omvandlarna behövs därför digital signalrekonstruktion. Konventionella algoritmer för detta problem tenderar att vara beräkningsintensiva, dvs de kräver många aritmetiska operationer (multiplikationer och additioner) för att korrigera varje sampel. Detta innebär en hög effektförbrukning om de ska implementeras i hårdvara.

Denna avhandling introducerar nya algoritmer för signalrekonstruktion i sammanflätade A/D-omvandlare. De föreslagna algoritmerna kräver betydligt färre aritmetiska operationer än befintliga lösningar. Därigenom kan 
samma höga prestanda för den sammanflätade A/D-omvandlaren uppnås, som för befintliga algoritmer, men med lägre beräknings- och implementeringskostnad. Avhandlingen behandlar både konventionella sammanflätade A/D-omvandlare, som beskrevs ovan, och okonventionella varianter, där en eller flera av de individuella omvandlarna inte används hela tiden eller inte alls.

Att inte använda omvandlarna hela tiden för den analoga insignalen kan utnyttjas för att utföra en robust estimering av mismatch-felen mellan de individuella omvandlarna, vilket behövs för att kunna designa en signalrekonstruktor. Detta sker genom att, i de tidsluckor som då skapas, applicera en känd signal för estimeringen. Att inte alls använda en del av de individuella omvandlarna kan utnyttjas för att dra fördel av strukturer i analoga signaler vilket möjliggör så kallad sub-Nykvist sampling och gles signalbehandling. Sub-Nykvist sampling av glesa signaler erbjuder kraftigt reducerad datatakt. Detta har potential att radikalt reducera effektförbrukningen för A/D-omvandlingen.

Avhandlingen omfattar både teori och designmetoder för ovan nämnda tillämpningar, samt många exempel som visar beräkningseffektiviteten hos de föreslagna algoritmerna. 


\section{Acknowledgments}

First and foremost, I would like to express my gratitude to my supervisor Professor Håkan Johansson for giving me an opportunity to perform the research that led to this dissertation. I have immensely benefited from the discussions with him and from the feedback and numerous insights that he provided during these four years. His invaluable guidance and encouragement has helped me to reach this far.

I would like to acknowledge the support provided by my former co-supervisor Dr. J. Jacob Wikner. I would also like to thank him for giving the Ph.D. course "Script-based IC design" which was one of the best Ph.D. courses that I have attended. I am very thankful to Dr. Oscar Gustafsson for all the support that he had extended to me while I was at the Division of Electronics Systems (ES). I also thank him for being the driving-force behind the ES weekly presentations which allowed me to hone my presentation skills as well as get relevant feedback on my work. I am also grateful to my co-supervisor Prof. Erik G. Larsson for granting me the opportunity to join the Division of Communication Systems (CommSys) and, for facilitating a stimulating research environment.

I wish to express my warmest thanks to Prakash Harikumar for being a very good friend and colleague. At times I have sought the help of his retentive memory which has always fascinated me. I also thank him for carefully proof-reading parts of this dissertation. Special thanks to my friend and colleague Vishnu Unnikrishnan for always being interested in discussing my work. I would also like to thank Dr. Hien Quoc Ngo for providing the $\mathrm{AT}_{\mathrm{E}} \mathrm{X}$ template which I have used to prepare this dissertation. Many thanks to all the former and current colleagues at ES and CommSys who have directly or indirectly helped me in my work. Working with you has been a privilege.

I take this opportunity to thank my wife Roshini for her encouragement and tremendous support which helped me to stay focused on my studies. I 
would also like to thank her parents, Ratnam and Hariprasad, for extending their help when it was needed the most. Their succor during a time of great personal loss allowed me to concentrate on my studies and, for this I am forever indebted to them. I am eternally grateful to my parents, Nirmala and Muralidharan, for their unconditional love and for supporting me in all the important decisions that I have taken in life.

It is also acknowledged that parts of the work which resulted in this dissertation were supported by the Swedish Research Council.

Linköping, May 2015

Anu Kalidas Muralidharan Pillai 


\title{
Abbreviations
}

\author{
ADC Analog-to-digital converter \\ ASIC Application-specific integrated circuit \\ CNUS Cyclic nonuniform sampling \\ DFT Discrete fourier transform \\ DMC Differentiator-multiplier cascade \\ DSP Digital signal processing \\ FB Filter bank \\ FD Fractional-delay \\ FIR Finite-length impulse response \\ IDFT Inverse discrete fourier transform \\ PR Perfect reconstruction \\ SFDR Spurious-free dynamic range \\ TI-ADC Time-interleaved analog-to-digital converter
}





\section{Contents}

$\begin{array}{ll}\text { Abstract } & \text { iii }\end{array}$

Populärvetenskaplig Sammanfattning (in Swedish) v

Acknowledgments vii

Abbreviations $\quad$ ix

I Introduction 1

1 Background 3

1.1 Preliminaries . . . . . . . . . . . . . . . . 6

1.1.1 Notations . . . . . . . . . . . . . . . . . . 6

1.1.2 Uniform Sampling . . . . . . . . . . . . . . . . . 6

$1.1 .3 \quad$ FIR Filters . . . . . . . . . . . . . . . . . . . . . 7

1.1 .4 Interpolation . . . . . . . . . . . . . . . 8

1.1.5 Decimation . . . . . . . . . . . . . . . . . 9 9

1.1.6 Polyphase Decomposition . . . . . . . . . . . . . . . . 10

1.1.7 Filter Banks . . . . . . . . . . . . . . . . . . . . 11

1.1.8 Cosine-Modulated Filter Banks . . . . . . . . . . . . . 12

1.1.9 Vandermonde Matrices . . . . . . . . . . . . . . . . . . 14

2 Mismatch Error Correction in TI-ADCs 15

2.1 Time-Interleaved ADCs . . . . . . . . . . . . . . 15

2.1.1 Static Time-Skew Errors in TI-ADCs . . . . . . . . 17

2.1.2 Channel Frequency Response Mismatches . . . . . . . 20

2.2 Time-Varying FIR Reconstructors . . . . . . . . . . . . . 21

2.3 Error Metrics and Reconstructor Design . . . . . . . . . . . 22

2.3.1 Least-Squares Design . . . . . . . . . . . . . . . . . . . 22

2.3 .2 Minimax Design . . . . . . . . . . . . . . . . . . . . . . 23

2.4 Reconstructor Complexity . . . . . . . . . . . . . . . . 24

2.5 Low-Complexity Reconstruction Schemes . . . . . . . . . 25

2.5.1 Two-Rate Based Approach _. . . . . . . . . . . . 26

2.6 Summary . . . . . . . . . . . . . . . . . . . . . . . . 29 
3 Sub-Nyquist Sampling of Sparse Multi-Band Signals 31

3.1 Sub-Nyquist Cyclic Nonuniform Sampling . . . . . . . . . . 32

3.2 Reconstruction Using Multi-Level Synthesis Filters . . . . . . 34

$3.2 .1 \quad$ Complexity . . . . . . . . . . . . . . . . . 37

3.3 Reconstruction Using Analysis and Synthesis Filters . . . . . 37

3.3 .1 Complexity . . . . . . . . . . . . . . . . 39

3.4 Summary and Future Extension . . . . . . . . . . . . . . . 40

4 Reconstruction in TI-ADCs with Missing Samples 41

4.1 TI-ADCs with Missing Samples . . . . . . . . . . . . . . . . 41

4.2 Reconstruction Schemes . . . . . . . . . . . . . . . . . 42

4.2.1 Constrained Time-Varying FIR Reconstructor . . . . . 43

4.2.2 Sub-Band Based Reconstructor . . . . . . . . . . . . 45

4.2.3 Pre-Filter Based Reconstructor . . . . . . . . . . . . . 46

4.2.4 Complexity Comparison . . . . . . . . . . . . . . 47

4.3 Noise Gain . . . . . . . . . . . . . . . . . . . . . 48

4.4 Summary . . . . . . . . . . . . . . . . . 51

5 Summary of Specific Contributions of the Dissertation 53

5.1 Included Papers . . . . . . . . . . . . . . . . . . 54

5.2 Not Included papers . . . . . . . . . . . . . . . . . 57

A Alternative Derivation of the Reconstruction Scheme in Paper D $\quad 59$

A.1 Lowpass Filters . . . . . . . . . . . . . . . . 60

A.2 Conventional Bandpass Filters . . . . . . . . . . . 62

A.3 Unconventional Bandpass Filters . . . . . . . . . . . . . . 64

B Derivation of the Least-Squares Design in Paper F 67

B.1 Constrained Time-Varying FIR Reconstructor . . . . . . . . . 67

B.2 Least-Squares Design of $F_{q}\left(e^{j \omega}\right)$ and $G_{q}\left(e^{j \omega}\right)$ in the Sub-band Based Reconstructor . . . . . . . . . . . . . . . . . 69

II Efficient Reconstruction Schemes for TI-ADCs 81

A Two-Rate Based Low-Complexity Time-Varying DiscreteTime FIR Reconstructors for Two-Periodic Nonuniformly Sampled Signals $\quad 83$ 1 Introduction . . . . . . . . . . . . . . . . . 86

2 Nonuniform Sampling and Time-Varying FIR Reconstructors 88

2.1 Reconstructors for Two-Channel TI-ADCs . . . . . . . 89 
3 Two-Rate Based Reconstructors _... . . . . . . . . . . . 91

3.1 Least-Squares Design . . . . . . . . . . . . . . . . . . 93

4 Polynomial Impulse Response FIR Reconstructors . . . . . . 97

5 Two-Rate Based Polynomial Impulse Response FIR Reconstructors . . . . . . . . . . . . . . . . . . . . 99

5.1 Least-Squares Design . . . . . . . . . . . . . . . 101

6 Design Example . . . . . . . . . . . . . . . . . . . 106

7 Conclusion . . . . . . . . . . . . . . . . . 110

B Efficient Signal Reconstruction Scheme for $M$-Channel Time-Interleaved ADCs 113

1 Introduction . . . . . . . . . . . . . . . 116

2 Background . . . . . . . . . . . . . . . . 118

$3 \quad M$-Channel Two-Rate Based Reconstructors . . . . . . . . . 121

3.1 Offline Design of $F_{0}(z) \ldots \ldots \ldots \ldots$

$3.2 \quad$ Online Design of $G_{0}^{(n)}(z)$ and $G_{1}^{(n)}(z) \ldots \ldots . \ldots 126$

4 Design Example . . . . . . . . . . . . . . . . . . 128

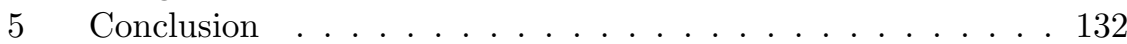

C Low-Complexity Two-Rate Based Multivariate Impulse Response Reconstructor for Time-Skew Error Correction in $M$-Channel Time-Interleaved ADCs 137

1 Introduction . . . . . . . . . . . . . . . . . . . . 140

$2 \quad M$-Periodic Nonuniform Sampling and Reconstruction . . . 142

3 Review of Multivariate Polynomial Impulse Response Reconstructors and Two-Rate Approach . . . . . . . . . . . . . . 143

3.1 Multivariate Polynomial Impulse Response Reconstructors . . . . . . . . . . . . . . . . . . . 143

3.2 Two-Rate Approach . . . . . . . . . . . . . . . . . . 144

4 Proposed Two-Rate Based Multivariate Polynomial Impulse Response Reconstructor . . . . . . . . . . . . . . . . . . . . 145

4.1 Reconstructor Design . . . . . . . . . . . . . 146

5 Design Example . . . . . . . . . . . . . . . . . . . . . . . 149

6 Conclusion . . . . . . . . . . . . . . . 150

III Reconstruction of Sub-Nyquist Sampled Sparse Multi-Band Signals

D Efficient Recovery of Sub-Nyquist Sampled Sparse MultiBand Signals Using Reconfigurable Multi-Channel Analysis and Modulated Synthesis Filter Banks 
1 Introduction . . . . . . . . . . . . . . . . 158

1.1 Contributions and Outline of the Paper . . . . . . 159

2 Preliminaries . . . . . . . . . . . . . . . . 162

2.1 Notations . . . . . . . . . . . . . . . . . 162

2.2 Polyphase Decomposition . . . . . . . . . . . . . . 162

2.3 Generalized Fractional-Delay Filter . . . . . . . . . . 163

3 Sub-Nyquist Cyclic Nonuniform Sampling of Sparse MultiBand Signals . . . . . . . . . . . . . . . . . . 163

4 Proposed Reconstruction Using Analysis and Synthesis FBs . 165

4.1 Unconventional Bandpass Filters . . . . . . . . . . . 165

4.2 Determining $\beta_{k m_{\ell}}$ and $\alpha_{k m_{\ell}} \ldots \ldots \ldots \ldots$

5 Proposed Efficient Reconstructor . . . . . . . . . . . . . . 172

5.1 Synthesis and Analysis FBs . . . . . . . . . . . . . 172

5.2 Computational Complexity . . . . . . . . . . . 173

5.3 Reconfiguration Complexity . . . . . . . . . . . . . 175

6 Design of the Proposed Reconstructor . . . . . . . . . . . 175

6.1 Prototype Filter Design . . . . . . . . . . . . 176

6.2 Least-Squares Design of $F_{\ell}(z)$ and $G_{\ell}(z) \ldots \ldots$. . 177

6.3 Design of Reconfigurable Reconstructors . . . . . . . . 179

6.4 Design Complexity . . . . . . . . . . . . . . . . . 180

$7 \quad$ Design Examples . . . . . . . . . . . . . . . . . . . . . 181

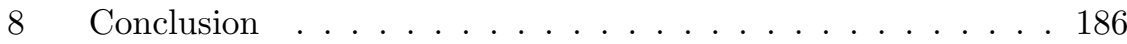

IV Reconstruction Schemes for TI-ADCs with Missing Samples

E A Sub-Band Based Reconstructor for $M$-Channel TimeInterleaved ADCs with Missing Samples 195

1 Introduction . . . . . . . . . . . . . . . . . . . . . . . 198

2 Prerequisites . . . . . . . . . . . . . . . . . . . . 199

3 Proposed Reconstructor . . . . . . . . . . . . . . . . . 200

$3.1 \quad$ Analysis Filters . . . . . . . . . . . . . . . . . . . 201

3.1.1 Reconfigurability . . . . . . . . . . . . 203

3.2 Synthesis Filters . . . . . . . . . . . . . . . . . . 204

3.3 Reconstructor Design . . . . . . . . . . . . . . 204

4 Design Example . . . . . . . . . . . . . . . . . 205

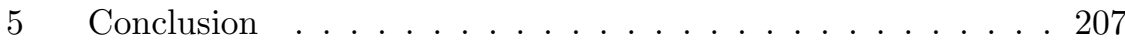

F Two Reconstructors for $M$-Channel Time-Interleaved ADCs with Missing Samples 211 1 Introduction . . . . . . . . . . . . . . . . . . . 214 
2 Background . . . . . . . . . . . . . . . . 215

3 Constrained Time-Varying FIR Reconstructor . . . . . . . . . 217

3.1 Least-Squares Design . . . . . . . . . . . . . . . . 218

4 Sub-Band Based Reconstructor . . . . . . . . . . . . . . . 218

4.1 Least-Squares Design . . . . . . . . . . . . . . . . . . . 221

5 Design Example . . . . . . . . . . . . . . . . . . 222

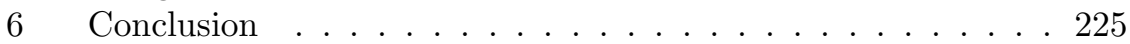

G Prefilter-Based Reconfigurable Reconstructor for TimeInterleaved ADCs with Missing Samples 229

1 Introduction . . . . . . . . . . . . . . . . . . 232

2 Background and Prerequisites . . . . . . . . . . . 233

3 Proposed Reconstructor . . . . . . . . . . . . . . . 235

3.1 Two-Mode Time-Varying Prefilter . . . . . . . . . . 235

3.2 Reconfigurable Part . . . . . . . . . . . . . . 236

3.3 Complexity . . . . . . . . . . . . . . . . . . . . . . 239

$3.4 \quad$ Design . . . . . . . . . . . . . . . . . . . . . . . 239

4 Design Examples . . . . . . . . . . . . . . . . . . . 241

5 Conclusion . . . . . . . . . . . . . . . . . 244 



\section{Part I}

\section{Introduction}





\section{Chapter 1}

\section{Background}

Today, digital signal processing (DSP) is extensively used in many applications. Advances in the field of microelectronics have made it possible to create efficient and cost-effective DSP hardware. Unlike analog electronics, digital systems are much less susceptible to manufacturing process variations as well as physical variations such as temperature changes. Moreover, deep sub-micron chip fabrication processes are well suited for digital circuits as the shrinking transistor sizes offer faster switching speeds and allow us to pack more digital logic in a given area. On the other hand, analog circuit design has become increasingly challenging especially in the deep sub-micron processes. All the above reasons have contributed towards the growing popularity of digital systems. However, many of these digital systems must interact with the analog world. For this purpose, analog-to-digital as well as digital-to-analog interfaces are frequently required at the input and output, respectively, of digital systems.

Typically, an analog-to-digital converter (ADC) samples a continuous-time analog signal at a predefined rate (sampling rate) to generate a discrete-time sequence of samples. The analog value of each sample is then represented using a finite number of bits (resolution). The sampling rate of the ADC is selected depending on the bandwidth of the analog input signal. There exist many ADC architectures that are suitable for different ranges of sampling rates and resolutions [1]. Analog-to-digital converters used in applications like communication systems and high-speed digitizers should support very high sampling rates and/or resolutions [2]. In such cases, implementing a single high-sampling rate $\mathrm{ADC}$ is quite challenging and at times infeasible. A 
popular technique to increase the effective sampling rate is to have multiple ADCs in a time-interleaved fashion with each ADC operating at a lower sampling rate [3].

In theory, by time-interleaving the outputs of $M$ channel ADCs, a timeinterleaved analog-to-digital converter (TI-ADC) can achieve the same resolution as that of the individual ADCs but with $M$ times higher sampling rate. However, in practice, the channel ADCs suffer from nonidealities such as gain, offset, and timing errors. These nonidealities manifest mainly due to analog circuit imperfections caused by variations in manufacturing process, voltage, and temperature $[4,5]$. Also, the reduced feature size of transistors in advanced manufacturing processes make within-die and die-to-die variations more pronounced due to the limited accuracy of the existing lithography techniques [6]. Due to the random nature of the variations [5-8], each channel ADC exhibits different levels of nonidealities which causes channel mismatch errors in TI-ADCs. In a TI-ADC with mismatch errors, the output is a nonuniformly sampled signal which degrades the achievable resolution at the output of the TI-ADC $[4,9]$. Thus, in order to retain the achievable resolution, TI-ADC implementations must either avoid mismatches between the channel ADCs through careful analog circuit design [7] or use calibration wherein the mismatch errors are estimated and then compensated for. The former approach is extremely challenging especially in newer digital-friendly chip manufacturing processes. Hence, TI-ADC implementations often rely on calibration to mitigate the effects of channel mismatch errors.

The mismatch errors can be broadly classified into linear and nonlinear mismatch errors [9-14]. Linear mismatch errors include gain, offset, timing skew, and frequency response mismatches whereas nonlinear mismatch errors occur due to mismatches in the nonlinearity of the channel ADCs. In this thesis we consider only linear mismatch errors which typically dominate [15]. More specifically, since gain and offset errors can be easily compensated, here we deal with only time-skew mismatch errors which occur as a result of nonuniform time skews between the sampling clocks of the channel ADCs [16-18]. In practice, the time-skew errors can be assumed to be frequency independent only up to a certain output resolution and bandwidth [19]. In high-speed TI-ADCs, the time-skew errors are frequency dependent $[20,21]$. Thus, to achieve very high resolutions, each channel in a high-speed TI-ADC is modeled as a general frequency response and the calibration block should compensate for the frequency-response mismatch errors. 
The calibration block utilizes the TI-ADC output to estimate the mismatch parameters which are then used to reconstruct the output so as to compensate for the mismatch errors. The reconstruction can be performed either in the analog domain [22,23] or in the digital domain [24]. Analog reconstructors use the mismatch estimates to perform the correction in the analog domain. Such reconstruction schemes are attractive for low power applications but they require significant design effort. Currently, such techniques appears only to be effective for low- to moderate-resolution ADCs as these techniques are more suited for correcting frequency-independent mismatch errors. On the other hand, digital reconstructors can be used to achieve arbitrarily high resolutions. Such reconstructors use the mismatch estimates to correct the samples at the output of the TI-ADC. However, these reconstructors require several computations per corrected output sample and hence, consume much power. Also, in TI-ADC implementations, the mismatch parameters can change from time to time. The reconstructor should be easily reconfigurable to cope with changes in the mismatch parameters. Thus, a challenge is to reduce the complexity of the reconstructor as well as to make it easily reconfigurable.

Following this introduction, in Part II of this thesis, we propose efficient signal reconstruction algorithms for conventional TI-ADCs. Here, we assume that mismatch parameters are estimated and are available beforehand. It is noted that efficient reconstruction schemes are also beneficial for estimation, for example, where calibration is performed using simultaneous estimation and compensation through the minimization of an appropriate cost measure. We also propose efficient reconstruction schemes for two unconventional TIADCs. In Part III, we consider an unconventional TI-ADC which is used for the sub-Nyquist cyclic nonuniform sampling of sparse multi-band signals [25]. In such TI-ADCs, only a few of the channel ADCs are active and, hence, the reconstructor should recover the uniform-grid samples from the available samples. In the second type of unconventional TI-ADC considered in Part IV, some of the ADC sampling instants are reserved for estimating the mismatch errors [26]. At these time instances, the input signal will not be sampled which results in missing samples at the output of the TI-ADC. Thus, the reconstructor used in such TI-ADCs should recover the missing samples and also correct for the mismatch errors.

Before proceeding to Part II, in Chapters 2, 3, and 4, we introduce some background materials and provide a brief overview of the thesis. Finally, Chapter 5 summarizes the specific contributions of the thesis. 


\section{$1.1 \quad$ Preliminaries}

In this section, after describing the notations, we will briefly review some of the basic theory and methods that are used throughout the thesis.

\subsubsection{Notations}

A continuous-time signal is denoted as $x(t)$ whereas $x(n)$ is used to denote a discrete-time signal. Here, $t$ represents the time axis and $n$ is the time index. Bold lowercase letters are used to denote vectors while bold uppercase letters are used to denote matrices. Transpose and conjugate-transpose are represented using $(\cdot)^{T}$ and $(\cdot)^{\dagger}$, respectively. For a filter with impulse response coefficients $h(n)$, we use $H(z)$ to denote its transfer function which is defined as $H(z)=\sum_{n} h(n) z^{-n}$. The frequency response of the filter is denoted by $H\left(e^{j \omega T}\right)$ and is obtained from the transfer function by replacing $z$ with $e^{j \omega T}$.

\subsubsection{Uniform Sampling}

Let $x_{a}(t)$ represent a continuous-time signal which is bandlimited to $\omega_{\mathrm{c}}<$ $\pi / T$ such that its Fourier transform $X_{a}(j \omega)$ defined by

$$
X_{a}(j \omega)=\frac{1}{2 \pi} \int_{-\infty}^{\infty} x_{a}(t) e^{-j \omega t} \mathrm{~d} t
$$

vanishes outside the interval $|\omega| \geq \omega_{\mathrm{c}}$. That is,

$$
X_{a}(j \omega)=0,|\omega| \geq \omega_{\mathrm{c}} .
$$

Then, according to the Nyquist sampling theorem [27-29], $x_{a}(t)$ can be reconstructed from a discrete-time signal $x(n)$ obtained by sampling $x_{a}(t)$ at time instances $t=n T$ where $T$ represents the sampling period. Using Poisson's summation formula with $x_{a}(t)$ bandlimited as in (1.2), the Fourier transform $X\left(e^{j \omega T}\right)$ of the uniform-sampling sequence $x(n)=x_{a}(n T)$ can be written as $[30]$

$$
X\left(e^{j \omega T}\right)=\frac{1}{T} X_{a}(j \omega), \omega T \in[-\pi, \pi] .
$$

Uniform sampling can be performed using a single ADC as shown in Fig. 1.1. The analog signal $x_{a}(t)$ is first uniformly sampled at time instants $t=n T$ after which the sampled value is quantized to a finite number of bits to form the uniform-sampling sequence $x(n)$. 


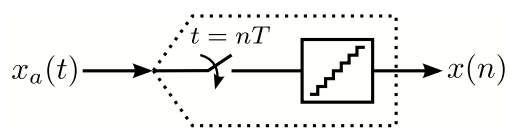

Figure 1.1: Uniform sampling using a single ADC.

\subsubsection{FIR Filters}

In a causal finite-length impulse response (FIR) filter of order $N$, the impulse response coefficients $h(n)$ can be nonzero only for $0 \leq n \leq N$ and are zero for all other values of $n$. Thus, its transfer function $H(z)$ is given by

$$
H(z)=\sum_{n=0}^{N} h(n) z^{-n} .
$$

In the case of a linear-phase FIR filter, the impulse response coefficients are symmetric or anti-symmetric. The frequency response of a linear-phase FIR filter can be expressed in terms of a real function $H_{\mathrm{R}}(\omega T)$ such that

$$
H\left(e^{j \omega T}\right)=H_{\mathrm{R}}(\omega T) e^{j \Phi(\omega T)}
$$

where

$$
\Phi(\omega T)=-\frac{N}{2} \omega T+c .
$$

In (1.6), $c=0$ if the filter coefficients $h(k)$ are symmetric and if the coefficients are anti-symmetric, $c=\pi / 2$. The real function $H_{\mathrm{R}}(\omega T)$ is called the zero-phase frequency response of $H\left(e^{j \omega T}\right)$ [31]. In the case of a noncausal linear-phase FIR filter, centered at $n=0, H\left(e^{j \omega T}\right)=H_{\mathrm{R}}(\omega T)$.

Let $x(n)$ and $y(n)$ represent the input and output, respectively, of a causal $N$ th-order FIR filter with impulse response $h(n)$. Then, the filtered output $y(n)$ is obtained by convolving $x(n)$ with $h(n)$ such that

$$
y(n)=\sum_{k=0}^{N} x(n-k) h(k) .
$$

It can be seen from (1.7) that in order to compute each output sample, an $N$ th-order FIR filter requires $N+1$ multiplications, $N$ additions, and $N$ delay elements. In a linear-phase FIR filter, due to the symmetry of the impulse response coefficients, we require only around $N / 2$ multipliers [31-33]. 


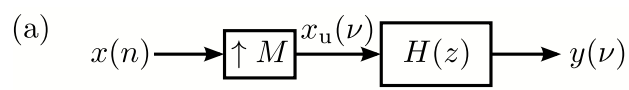

(b)
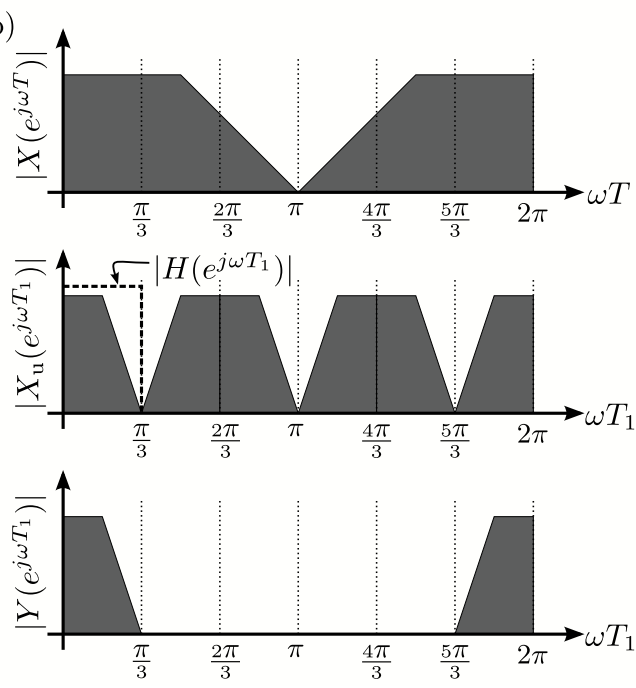

Figure 1.2: (a) Interpolation by $M$. (b) Example spectrum of the input, upsampled, and interpolated sequences with interpolation factor $M=3$ and an ideal filter $H(z)$. Here, $T_{1}=T / 3$.

\subsubsection{Interpolation}

Interpolation is the process of increasing the sampling frequency of a sequence $x(n)$. As shown in Fig. 1.2(a), interpolation by a factor of $M$ involves upsampling by $M$ followed by filtering. The upsampler inserts $M-1$ zeros between the samples in $x(n)$ such that the upsampled sequence $x_{\mathrm{u}}(\nu)$ becomes

$$
x_{\mathrm{u}}(\nu)= \begin{cases}x(n), & \nu=n M \\ 0, & \text { otherwise. }\end{cases}
$$

If $X(z)$ represents the $z$-transform of $x(n)$, then the $z$-transform of $x_{\mathrm{u}}(n)$ is given by

$$
X_{\mathrm{u}}(z)=X\left(z^{M}\right) .
$$

Thus, due to upsampling, $M-1$ image terms appear in the frequency band $\omega T \in[0,2 \pi)$. These unwanted images are removed using the interpolation filter $H(z)$ and we obtain the interpolated output $y(n)$. Figure 1.2(b) shows an example spectrum of $x(n), x_{\mathrm{u}}(\nu)$, and $y(\nu)$ when the input $x(n)$ is interpolated by a factor of three $(M=3)$ using an ideal interpolation filter $H(z)$. 
(a)

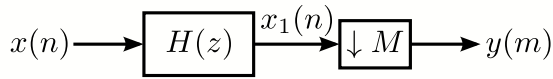

(b)
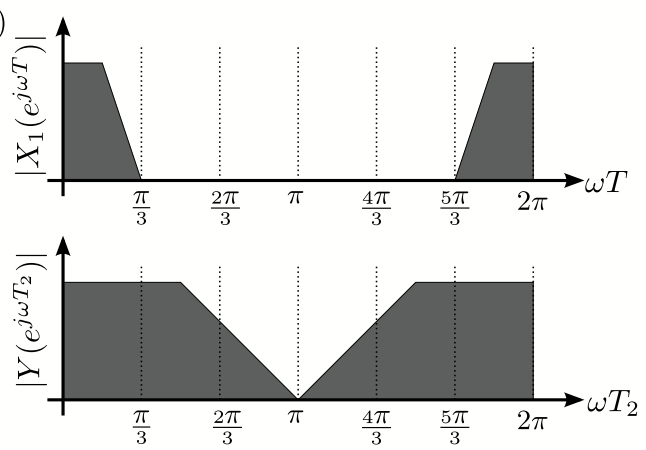

Figure 1.3: (a) Decimation by $M$. (b) An example spectrum of $x_{1}(n)$ and the spectrum of the corresponding decimated sequence $y(m)$ when the decimation factor is $M=3$. Here, $T_{2}=3 T$.

Note that the passband gain of $H(z)$ should be equal to $M$ to preserve the signal power.

\subsubsection{Decimation}

Decimation is the process of reducing the sampling rate of a sequence. Decimation by a factor of $M$ involves decimation filtering followed by downsampling by $M$ as shown in Fig. 1.3(a) [32,33]. The downsampler block shown in Fig. 1.3(a) is used to perform the downsampling operation wherein the downsampled sequence $y(m)$ contains only every $M$ th sample in $x_{1}(n)$. That is,

$$
y(m)=x_{1}(m M) .
$$

The $z$-transform of the downsampled sequence $y(m)$ is given by

$$
Y(z)=\frac{1}{M} \sum_{q=0}^{M-1} X_{1}\left(z^{1 / M} e^{-j 2 \pi q / M}\right) .
$$

In the summation in $(1.11)$, the terms $q=1,2, \ldots, M-1$, correspond to $M-1$ aliasing terms. Hence, to prevent aliasing into the signal band, a decimation filter $H(z)$ is used to bandlimit $x(n)$ to $\pi / M$ as shown in Fig. 1.3(a). As illustrated for $M=3$ in Fig. 1.3(b), with $x_{1}(n)$ bandlimited to $\pi / M$, aliasing terms due to the downsampler will not fall into the signal band. 


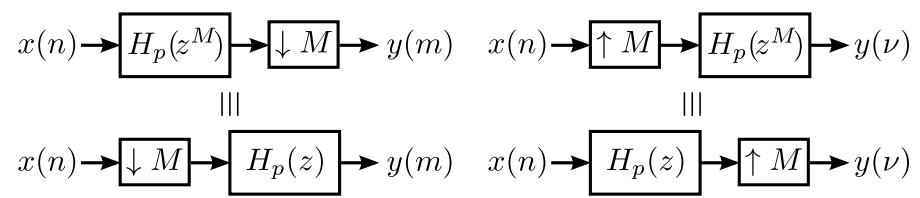

(a)

(b)

Figure 1.4: Noble identities.

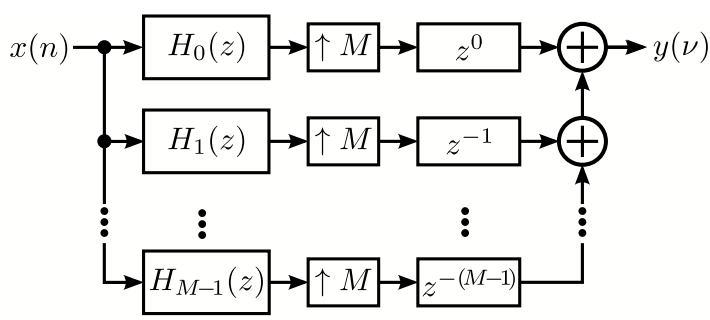

Figure 1.5: Equivalent representation of the interpolator in Fig. 1.2(a) using the $M$ polyphase branches of the filter $H(z)$.

\subsubsection{Polyphase Decomposition}

Any filter $H(z)$ can generally be expressed in terms of its polyphase components $H_{p}(z), p=0,1, \ldots, M-1$, as $[32,34]$

$$
H(z)=\sum_{p=0}^{M-1} z^{-p} H_{p}\left(z^{M}\right) .
$$

Polyphase decomposition as in (1.12) along with the noble identities shown in Fig. 1.4 [32], can be used to derive efficient structures for interpolation and decimation. For example, consider the interpolator shown in Fig. 1.2(a). Expressing $H(z)$ in Fig. 1.2(a) as in (1.12) and then propagating the upsampler to the right using the noble identity shown in Fig. 1.4(b), we get the polyphase structure in Fig. 1.5. It can be seen that, unlike in Fig. 1.2(a), in the polyphase structure the filtering takes place at the lower rate. In practice, the upsamplers, delays, and additions in Fig. 1.5 can be replaced with a commutator [32]. 


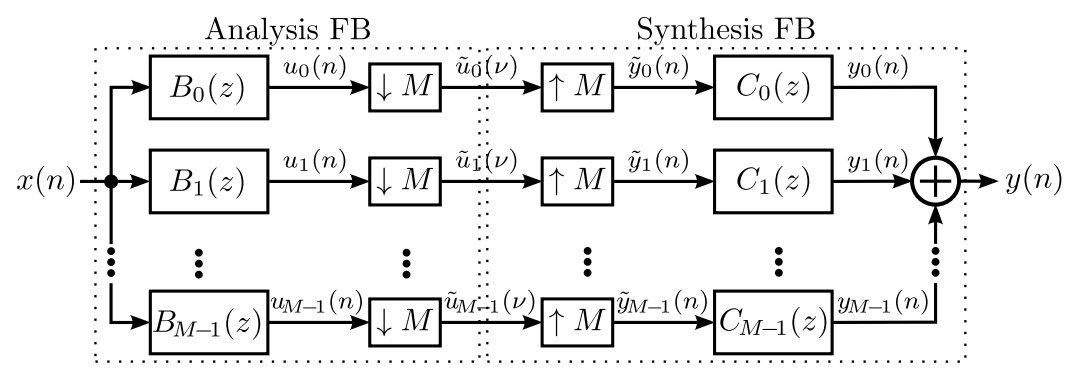

Figure 1.6: $M$-channel maximally decimated filter bank.

\subsubsection{Filter Banks}

Consider the $M$-channel maximally decimated filter bank (FB) shown in Fig. $1.6[32,35]$. The $z$-transform of the output of the analysis filter $B_{k}(z)$, $k \in 0,1, \ldots, M-1$, can be written as

$$
U_{k}(z)=B_{k}(z) X(z)
$$

where $X(z)$ is the $z$-transform of the input $x(n)$. Using (1.13), the $z$ transform of the corresponding decimated output $\tilde{u}_{k}(\nu)$ can be written as

$$
\begin{aligned}
\widetilde{U}_{k}(z) & =\frac{1}{M} \sum_{q=0}^{M-1} U_{k}\left(z^{1 / M} e^{-j 2 \pi q / M}\right) \\
& =\frac{1}{M} \sum_{q=0}^{M-1} B_{k}\left(z^{1 / M} e^{-j 2 \pi q / M}\right) X\left(z^{1 / M} e^{-j 2 \pi q / M}\right) .
\end{aligned}
$$

Since, $\tilde{y}_{k}(n)$ is obtained by upsampling $\tilde{u}_{k}(\nu)$ by $M$, its $z$-transform $\tilde{Y}_{k}(z)$ is given by

$$
\widetilde{Y}_{k}(z)=\widetilde{U}_{k}\left(z^{M}\right)=\frac{1}{M} \sum_{q=0}^{M-1} B_{k}\left(z e^{-j 2 \pi q / M}\right) X\left(z e^{-j 2 \pi q / M}\right) .
$$

The $z$-transform of $y_{k}(n)$ can be expressed using (1.15) as

$$
Y_{k}(z)=\frac{1}{M} C_{k}(z) \sum_{q=0}^{M-1} B_{k}\left(z e^{-j 2 \pi q / M}\right) X\left(z e^{-j 2 \pi q / M}\right) .
$$


Then, the $z$-transform of the output $Y(z)$ is given by

$$
Y(z)=\sum_{k=0}^{M-1} Y_{k}(z)=V_{0}(z) X(z)+\sum_{q=1}^{M-1} V_{q}(z) X\left(z e^{-j 2 \pi q / M}\right)
$$

where

$$
V_{q}(z)=\frac{1}{M} \sum_{k=0}^{M-1} C_{k}(z) B_{k}\left(z e^{-j 2 \pi q / M}\right)
$$

for $q=0,1, \ldots, M-1$. In (1.17), $V_{0}(z)$ is the distortion function and $V_{q}(z)$, $q=1,2, \ldots, M-1$, are the $M-1$ aliasing terms. It can be seen from (1.17) that with $V_{0}(z)=1$ and $V_{q}(z)=0$ for $q=1,2, \ldots, M-1$, we have perfect reconstruction $(\mathrm{PR})$. That is, $y(n)=x(n)$.

\subsubsection{Cosine-Modulated Filter Banks}

Approximate PR can be achieved by representing the impulse response coefficients of the analysis and synthesis filters in terms of a power-symmetric lowpass prototype filter $P(z)$ with cutoff frequency at $\pi / 2 M$, according to

$$
b_{k}(n)=2 p(n) \cos \left[\frac{\pi}{M}(k+0.5)\left(n-\frac{N}{2}\right)+(-1)^{k} \frac{\pi}{4}\right]
$$

and

$$
c_{k}(n)=2 p(n) \cos \left[\frac{\pi}{M}(k+0.5)\left(n-\frac{N}{2}\right)-(-1)^{k} \frac{\pi}{4}\right]
$$

where $p(n)$ represents the impulse response coefficient of $P(z)$ with filter order $N$. Expressing the analysis and synthesis filters as in (1.19) and (1.20) allows us to implement the FB in Fig. 1.6 using the efficient structure in Fig. 1.7 [35]. In Fig. 1.7, the $P_{q}(z), q=0,1, \ldots, 2 M-1$, are the $2 M$ polyphase components of $P(z)$ such that

$$
P(z)=\sum_{q=0}^{2 M-1} z^{-q} P_{q}\left(z^{2 M}\right)
$$

The complexity of the cosine-modulation block in the analysis FB (synthesis FB) in Fig. 1.7 can be reduced by using a fast-transform algorithm $[36,37]$ whereas for the prototype filter, only $N / M$ multiplications per input/output sample are required. 


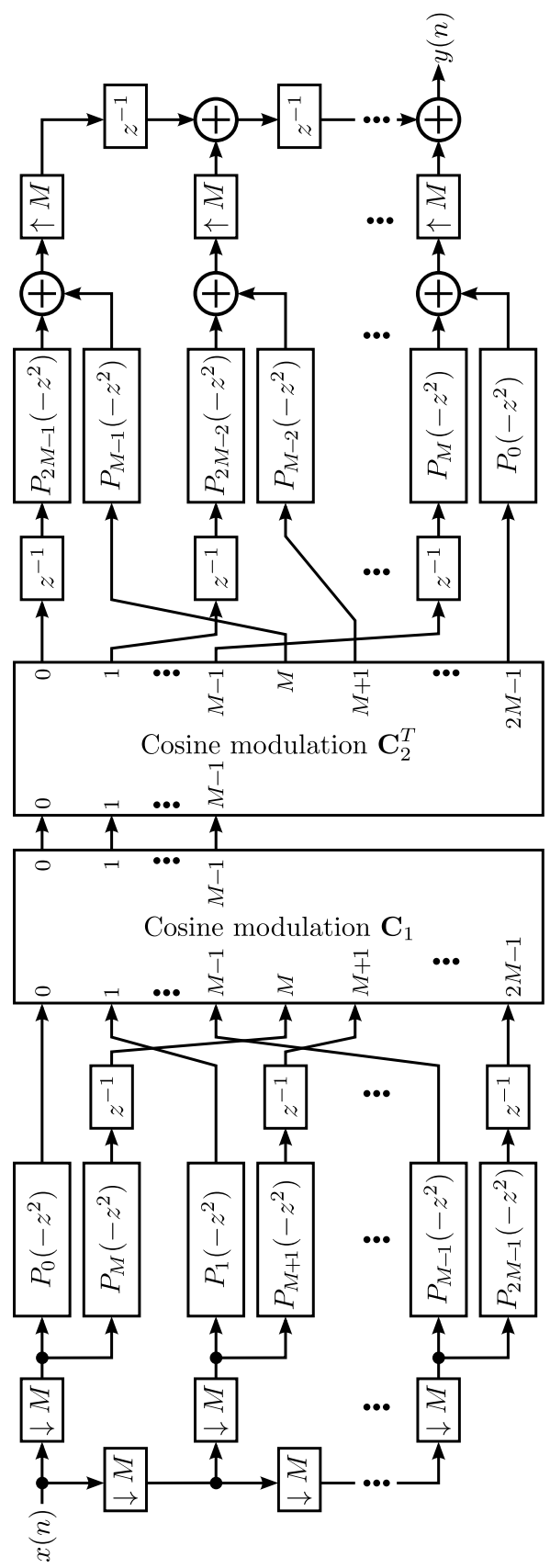

Figure 1.7: Efficient realization of the filter bank in Fig. 1.6 using a cosinemodulated FB. 


\subsubsection{Vandermonde Matrices}

A $(K+1) \times(K+1)$ matrix $\mathbf{V}$ is a Vandermonde matix if it has the form $[38,39]$

$$
\mathbf{V}=\left[\begin{array}{cccc}
1 & 1 & \cdots & 1 \\
x_{1} & x_{2} & \cdots & x_{K} \\
x_{1}^{2} & x_{2}^{2} & \cdots & x_{K}^{2} \\
\vdots & \vdots & \ddots & \vdots \\
x_{1}^{K} & x_{2}^{K} & \cdots & x_{K}^{K}
\end{array}\right] .
$$

The Vandermonde matrix is invertible if and only if the values $x_{k}, k=$ $1,2, \ldots, K-1$, in (1.22) are distinct [32]. The transpose of $\mathbf{V}$ is also a Vandermonde matrix.

A generalized $(K+1) \times(K+1)$ Vandermonde matrix has the form [38]

$$
\mathbf{V}=\left[\begin{array}{cccc}
a_{1}^{b_{1}} & a_{2}^{b_{1}} & \cdots & a_{K}^{b_{1}} \\
a_{1}^{b_{2}} & a_{2}^{b_{2}} & \cdots & a_{K}^{b_{2}} \\
\vdots & \vdots & \ddots & \vdots \\
a_{1}^{b_{K}} & a_{2}^{b_{K}} & \cdots & a_{K}^{b_{K}}
\end{array}\right] .
$$




\section{Chapter 2}

\section{Mismatch Error Correction in TI-ADCs}

This chapter provides an overview of Part II of the thesis where we consider reconstruction schemes for conventional TI-ADCs.

\subsection{Time-Interleaved ADCs}

Analog-to-digital converters supporting very high sampling rates often use time-interleaving of multiple ADCs to reduce the requirements on the individual ADCs [40]. In an $M$-channel TI-ADC, the continuous-time signal $x_{a}(t)$ is sampled using $M$ parallel ADCs as shown in Fig. 2.1(a) [3]. The sampling clocks to the channel ADCs are applied in such a way that, at any given time instant, only one channel ADC samples the input. In an ideal TI-ADC, the $m$ th channel ADC samples the input $x_{a}(t)$ as shown in Fig. 2.1(b). ${ }^{1}$ In this case, since $x_{m}(\nu)=x_{a}(\nu M T+m T)=x(\nu M+m)$, the output of the $m$ th channel ADC $x_{m}(\nu)$ can be considered as being obtained from the uniform-sampling sequence $x(n)$ as shown in Fig. 2.1(c) where $z=e^{j \omega T}$. Hence, as discussed in Section 1.1.5, the Fourier transform of the downsampled sequence $x_{m}(\nu)$ can be written as

$$
X_{m}\left(e^{j \omega T}\right)=\frac{1}{M} \sum_{k=0}^{M-1} e^{j \frac{\omega T-2 \pi k}{M} m} X\left(e^{j \frac{\omega T-2 \pi k}{M}}\right) .
$$

\footnotetext{
${ }^{1}$ To simplify the TI-ADC model, in Fig. 2.1 we have ignored the quantizer block which follows the sampler in the channel ADC.
} 
(a)

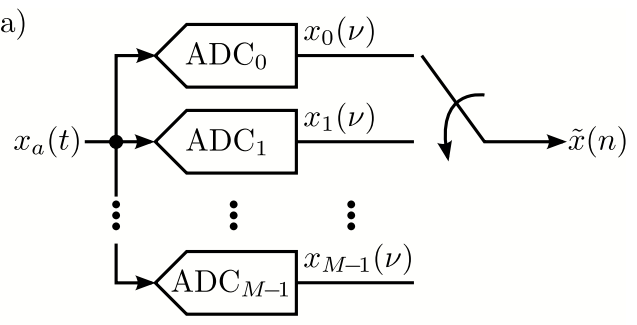

(b)

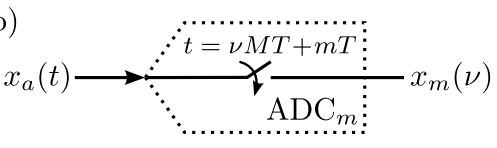

$x_{a}(t) \stackrel{t=n T}{x(n)} \underset{z^{m} \rightarrow \downarrow M}{\longrightarrow} \rightarrow x_{m}(\nu)$

Figure 2.1: (a) Ideal $M$-channel TI-ADC. (b) Block diagram of the $m$ th channel ADC. (c) Equivalent representation of (b).

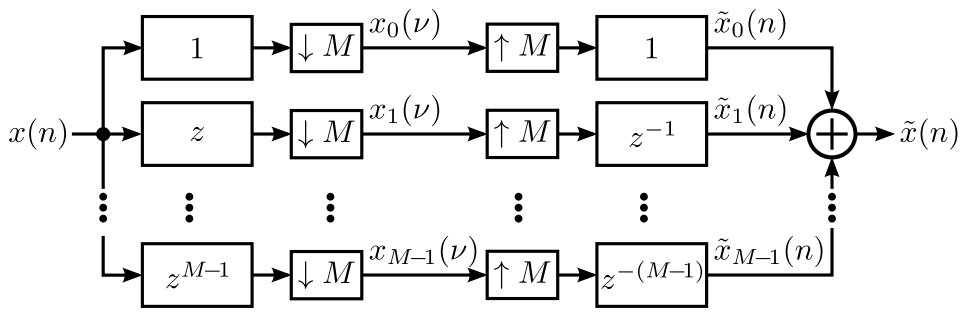

Figure 2.2: Equivalent multirate representation of the ideal $M$-channel TI$\mathrm{ADC}$ in Fig. 2.1(a). 
Now, the interleaved output $\tilde{x}(n)$ in Fig. 2.1(a) can be expressed as

$$
\tilde{x}(n)=\sum_{m=0}^{M-1} \tilde{x}_{m}(n)
$$

where $\tilde{x}_{m}(n), m=0,1, \ldots, M-1$, are obtained as shown in Fig. 2.2. Let $\widetilde{X}_{m}\left(e^{j \omega T}\right)$ represent the Fourier transform of $\tilde{x}_{m}(n)$. Then, using (2.1), we get

$$
\begin{aligned}
\tilde{X}_{m}\left(e^{j \omega T}\right) & =e^{-j \omega m T} X_{m}\left(e^{j \omega T M}\right) \\
& =\frac{1}{M} \sum_{k=0}^{M-1} e^{-j \frac{2 \pi k}{M} m} X\left(e^{j\left(\omega T-\frac{2 \pi k}{M}\right)}\right) .
\end{aligned}
$$

Now, taking the Fourier transform on both sides of the equality in (2.2) and using (2.3), we obtain

$$
\widetilde{X}\left(e^{j \omega T}\right)=\frac{1}{M} \sum_{k=0}^{M-1} X\left(e^{j\left(\omega T-\frac{2 \pi k}{M}\right)}\right) \sum_{m=0}^{M-1} e^{-j \frac{2 \pi k}{M} m}=X\left(e^{j \omega T}\right) .
$$

The second equality in (2.4) follows from $\sum_{m=0}^{M-1} \exp (-j 2 \pi k m / M)=0$ for $k \neq 0$. Thus, the continuous-time input $x_{a}(t)$ is uniformly sampled by the TI-ADC if the sampling clocks to the channel ADCs are applied as shown in Fig. 2.1(b). Hence, the time-skew between the sampling clocks of any two adjacent $\mathrm{ADCs}, \mathrm{ADC}_{m}$ and $\mathrm{ADC}_{m+1}$, should be equal to $T$. However, in practice, due to mismatches in the channel ADCs and clock routing network, the time-skew between the adjacent channels will not be uniform, resulting in a nonuniform-sampling sequence $v(n)$ at the output of the TI-ADC.

\subsubsection{Static Time-Skew Errors in TI-ADCs}

At moderate sampling rates and resolutions, the time-skew error can be approximated as static which means that the time-skews are frequencyindependent [19]. In this case, the nonuniform-sampled sequence $v(n)$ can be written as

$$
v(n)=x_{a}\left(n T+\varepsilon_{n} T\right)
$$

where $\varepsilon_{n}$ is the percentage deviation of the $n$th sample from the desired sampling instant $n T$. Using (1.2) and (1.3), we can rewrite $v(n)$ using the inverse Fourier transform of $x(n)$ as

$$
v(n)=\frac{1}{2 \pi} \int_{-\omega_{\mathrm{c}} T}^{\omega_{\mathrm{c}} T} e^{j \omega T \varepsilon_{n}} X\left(e^{j \omega T}\right) e^{j \omega T n} \mathrm{~d}(\omega T) .
$$



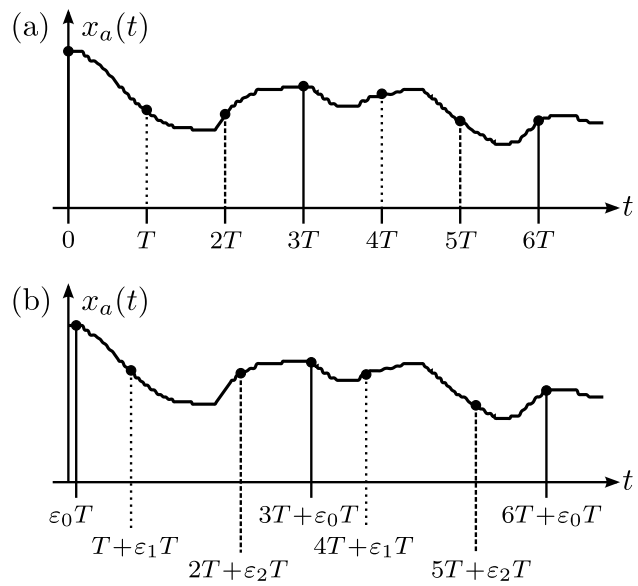

Figure 2.3: (a) Uniform sampling of $x_{a}(t)$ in an ideal three-channel TI-ADC. (b) Three-periodic nonuniform sampling of $x_{a}(t)$ in a three-channel TI-ADC with time-skew errors.

Since the output samples in a TI-ADC are formed by interleaving the outputs from each channel ADC, the deviations (time-skew errors) are periodic. In an $M$-channel TI-ADC, the time-skew errors are $M$-periodic such that

$$
\varepsilon_{n}=\varepsilon_{n+M} .
$$

For example, as shown in Fig. 2.3(b), the time-skew errors in a three-channel TI-ADC will be three-periodic with $\varepsilon_{3 n}=\varepsilon_{0}, \varepsilon_{3 n+1}=\varepsilon_{1}$, and $\varepsilon_{3 n+2}=\varepsilon_{2}$. Figure 2.4 shows the effect of time-skew errors in a three-channel TI-ADC. In Fig. 2.4(a), $x(n)$ is a uniform-sampling sequence at the output of the ideal three-channel TI-ADC and $v(n)$ is the corresponding TI-ADC output sequence when the channel ADCs have static time-skew errors. Figure 2.4(b) shows the amplitude spectrum of $x(n), v(n)$, and a reconstructed sequence. It can be seen that, due to time-skew errors, aliasing terms appear at the output of the TI-ADC, degrading the achievable resolution. As illustrated in Fig. 2.4(b), a reconstructor can be used to suppress the aliasing terms at the output of the TI-ADC. It is noted that, using an appropriate filter order for the reconstructor, the aliasing terms can be suppressed even further. Figure 2.5 shows the plot of spurious-free dynamic range (SFDR) versus $\varepsilon_{\max }$ for a two-channel TI-ADC with time-skew errors $\varepsilon_{0,1}=\left[\varepsilon_{\max },-\varepsilon_{\max }\right]$ and where the input is bandlimited to $|\omega T| \leq 0.9 \pi$. It can be seen that, as the magnitude of the time-skew errors increases, the achievable resolution at the output of the TI-ADC decreases. 

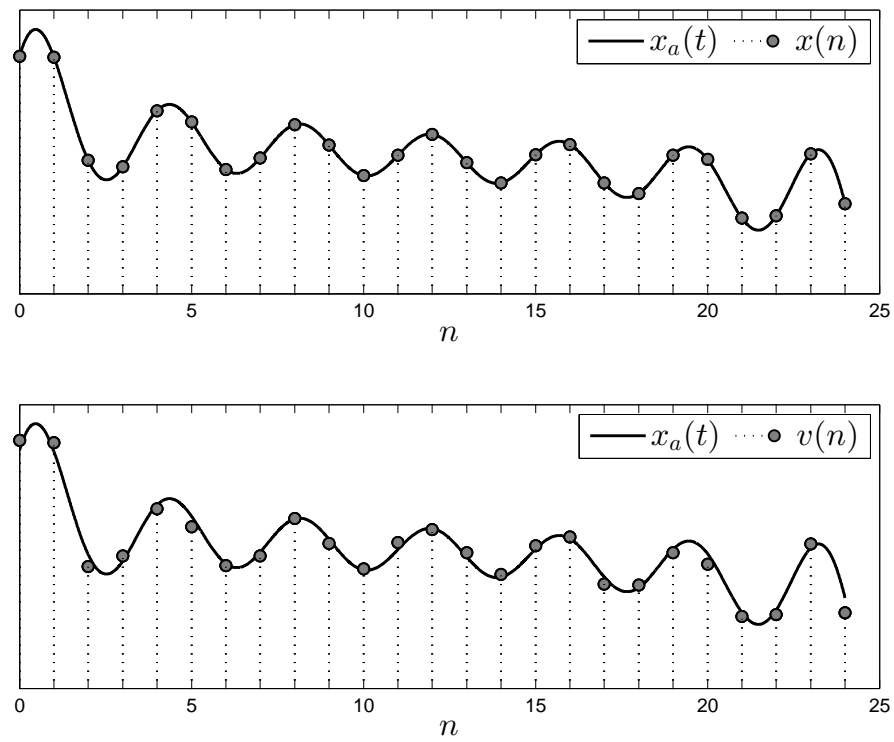

(a) Time-domain signals
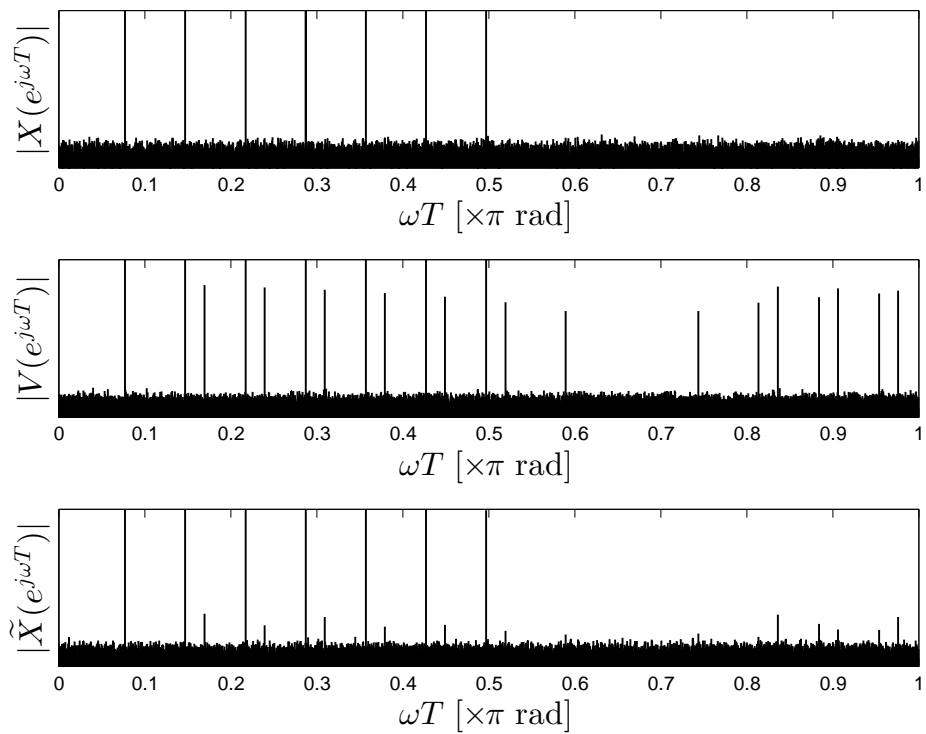

(b) Amplitude spectrum of the uniform-sampling (top), nonuniformsampling (middle), and reconstructed (bottom) sequences.

Figure 2.4: Time-domain signals and the corresponding amplitude spectra at the output of an ideal and a nonideal three-channel TI-ADC. In the nonideal TI-ADC, the channel ADCs have static time-skew errors. 


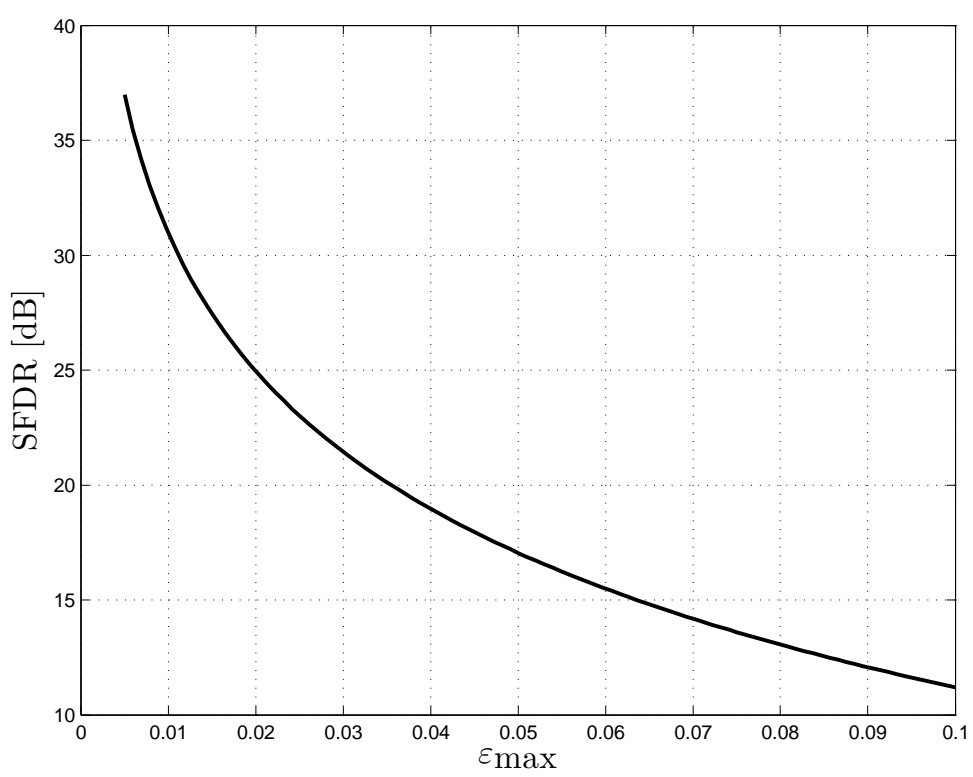

Figure 2.5: SFDR vs. $\varepsilon_{\max }$ in a two-channel TI-ADC with time-skew errors $\varepsilon_{0,1}=\left[\varepsilon_{\max },-\varepsilon_{\max }\right]$ and when the input is bandlimited to $|\omega T| \leq 0.9 \pi$.

\subsubsection{Channel Frequency Response Mismatches}

In high-speed TI-ADCs supporting very high resolutions, the TI-ADC model should be extended to include the channel frequency responses $Q_{m}(j \omega), m=$ $0,1, \ldots, M-1$, as shown in Fig. $2.6[11,20,41]$. Here, assuming that the continuous-time input $x_{a}(t)$ is bandlimited as in (1.2), the TI-ADC output $v(n)$ can be considered as obtained by sampling the output of a time-varying continuous-time system such that

$$
v(n)=\frac{1}{2 \pi} \int_{-\omega_{\mathrm{c}} T}^{\omega_{\mathrm{c}} T} Q_{n}(j \omega) X\left(e^{j \omega T}\right) e^{j \omega T n} \mathrm{~d}(\omega T)
$$

where $Q_{n}(j \omega)=Q_{n+M}(j \omega), \forall n \in \mathbb{Z}$, and $X\left(e^{j \omega T}\right)$ represents the Fourier transform of the uniform-sampling sequence as in (1.3). It can be seen that with $Q_{n}(j \omega)=e^{j \omega T \varepsilon_{n}},(2.8)$ reduces to $v(n)=x_{a}\left(n T+\varepsilon_{n} T\right)$ which corresponds to the nonuniform-sampling sequence at the output of a TIADC with static time-skew errors. 


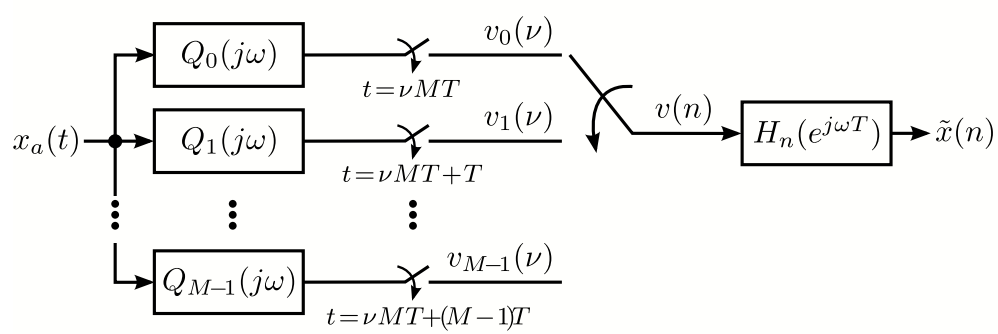

Figure 2.6: Model of an $M$-channel TI-ADC with $M$ different channel frequency responses $Q_{m}(j \omega), m=0,1, \ldots, M-1$, and a time-varying reconstructor $H_{n}\left(e^{j \omega T}\right)$.

\subsection{Time-Varying FIR Reconstructors}

In TI-ADC implementations, a digital reconstructor $H_{n}\left(e^{j \omega T}\right)$ can be used to recover the uniform-sampling sequence $x(n)$ from the nonuniformsampling sequence $v(n)$ as shown in Fig. 2.6 [41-56]. The reconstructor is a time-varying FIR filter whose impulse-response coefficients $h_{n}(k)$, are determined such that the reconstructed output $\tilde{x}(n)$, given by

$$
\tilde{x}(n)=\sum_{k=-N / 2}^{N / 2} v(n-k) h_{n}(k),
$$

approximates $x(n)$ [57]. Here, $N$ is the order of the reconstructor. We assume noncausal even-order filters to simplify the design and analysis. ${ }^{2}$ Since, the input of an $M$-channel TI-ADC with mismatches, is $M$-periodically nonuniformly sampled [57-59], the impulse response coefficients of the reconstructor are also $M$-periodically time-varying. That is, $h_{n}(k)=h_{n+M}(k)$. Further, in practice, the channel mismatch errors are estimated by using one of the channel ADCs as a reference channel. Due to this, the samples from the reference channel require no correction and, hence, $h_{n}(k)$ for the reference channel is a pure delay. Thus, for an $M$-channel TI-ADC, the time-varying FIR reconstructor can be realized using $M-1$ separate FIR filters [57]. It is noted that $h_{n}(k)$ is centered at the sample to be reconstructed.

2 The filters can be easily converted to causal filters by introducing suitable delays. Further, with minor modifications, all the derivations can be applied to the odd-order case as well. 
Substituting (2.8) in (2.9) and rearranging the terms, we get

$$
\tilde{x}(n)=\frac{1}{2 \pi} \int_{-\omega_{\mathrm{c}} T}^{\omega_{\mathrm{c}} T} A_{n}(j \omega) X\left(e^{j \omega T}\right) e^{j \omega T n} \mathrm{~d}(\omega T)
$$

where

$$
A_{n}(j \omega)=\sum_{k=-N / 2}^{N / 2} h_{n}(k) Q_{n-k}(j \omega) e^{-j \omega T k} .
$$

Further, if $h_{n}(k)$ perfectly reconstructs the uniform-grid samples, then $\tilde{x}(n)=x(n)$. Recall that, using the inverse Fourier transform of $X\left(e^{j \omega T}\right)$ $[60,61]$, we have

$$
x(n)=\frac{1}{2 \pi} \int_{-\omega_{\mathrm{c}} T}^{\omega_{\mathrm{c}} T} X\left(e^{j \omega T}\right) e^{j \omega T n} \mathrm{~d}(\omega T) .
$$

Thus, comparing (2.10) and (2.12) we see that to obtain perfect reconstruction, we require

$$
A_{n}(j \omega)=1, \omega T \in\left[-\omega_{\mathrm{c}} T, \omega_{\mathrm{c}} T\right] .
$$

However, in practice, perfect reconstruction $(\mathrm{PR})$ is not feasible with realizable filters and, moreover, is not a requirement in TI-ADC implementations. Thus, it is sufficient to determine $h_{n}(k)$ so that $A_{n}(j \omega)$ approximates unity in such a way that the reconstruction error $e(n)=\tilde{x}(n)-x(n)$ is minimized according to a specified error metric.

\subsection{Error Metrics and Reconstructor Design}

\subsubsection{Least-Squares Design}

The impulse response coefficients $h_{n}(k), n \in[0,1, \ldots, M-1]$, can be determined such that $A_{n}(j \omega), n \in[0,1, \ldots, M-1]$, approximates unity in the least-squares sense. Here, the aim is to minimize the energy of the error between the desired and the reconstructed sequences. For this purpose, we use the error power functions $P_{n}$ given by [57]

$$
P_{n}=\frac{1}{2 \pi} \int_{-\omega_{\mathrm{c}} T}^{\omega_{\mathrm{c}} T}\left|A_{n}(j \omega)-1\right|^{2} \mathrm{~d}(\omega T) .
$$


Let $\mathbf{h}_{n}$ represent the vector $((N+1) \times 1$ matrix $)$ containing the $N+1$ impulse response coefficients of $h_{n}(k)$ such that

$$
\mathbf{h}_{n}=\left[h_{n}(-N / 2) h_{n}(-N / 2+1) \ldots h_{n}(N / 2)\right]^{T} .
$$

Then, (2.14) can be written in matrix form as

$$
\mathrm{P}_{n}=\mathbf{h}_{n}^{T} \mathbf{S}_{n} \mathbf{h}_{n}-2 \mathbf{b}_{n}^{T} \mathbf{h}_{n}+\frac{\omega_{\mathrm{c}} T}{\pi}
$$

where $\mathbf{S}_{n}$ is an $(N+1) \times(N+1)$ matrix with elements $S_{n, k p}, k, p=$ $-N / 2,-N / 2+1, \ldots, N / 2$, given by

$$
\begin{aligned}
S_{n, k p}= & \frac{1}{2 \pi} \int_{-\omega_{\mathrm{c}} T}^{\omega_{\mathrm{c}} T}\left|Q_{n-k}(j \omega)\right|\left|Q_{n-p}(j \omega)\right| \\
& \times \cos \left(\omega(p-k) T+\arg \left\{Q_{n-k}(j \omega)\right\}-\arg \left\{Q_{n-p}(j \omega)\right\}\right) \mathrm{d}(\omega T)
\end{aligned}
$$

and

$$
\mathbf{b}_{n}=\left[\begin{array}{llll}
b_{n,-N / 2} & b_{n,-N / 2+1} & \ldots & b_{n, N / 2}
\end{array}\right]^{T}
$$

with

$$
b_{n, k}=\frac{1}{2 \pi} \int_{-\omega_{\mathrm{c}} T}^{\omega_{\mathrm{c}} T}\left|Q_{n-k}(j \omega)\right| \cos \left(\omega T-\arg \left\{Q_{n-k}(j \omega)\right\}\right) \mathrm{d}(\omega T)
$$

for $k=-N / 2,-N / 2+1, \ldots, N / 2$. The value of $\mathbf{h}_{n}$ that minimizes $\mathrm{P}_{n}$ in (2.16) is obtained by solving

$$
\frac{\partial \mathrm{P}_{n}}{\partial \mathbf{h}_{n}}=0
$$

which gives

$$
\mathbf{h}_{n}=\mathbf{S}_{n}^{-1} \mathbf{b}_{n}^{T}
$$

Thus, using a least-squares approach, the coefficients of each $N$ th-order FIR reconstructor $h_{n}(k)$ can be determined through an $(N+1) \times(N+1)$ matrix inversion.

\subsubsection{Minimax Design}

The SFDR of an ADC is a crucial metric especially in communication applications [62]. In such applications, the reconstructor is required to ensure that the maximum amplitudes of the spurious frequency components due to 
nonuniform sampling, are kept below a specified level. In such cases, $h_{n}(k)$, $n=0,1, \ldots, M-1$, can be determined such that $A_{n}(j \omega)$ approximates unity in the minimax sense. However, a more appropriate measure is obtained by using a distortion function $V_{0}\left(e^{j \omega T}\right)$ and $M-1$ aliasing functions $V_{m}\left(e^{j \omega T}\right)$, $m=1,2, \ldots, M-1$. Using these functions, the input-output relation of the reconstructor in the frequency domain can be written as (see (1.17) in Section 1.1.7) [57]

$$
\widetilde{X}\left(e^{j \omega T}\right)=V_{0}\left(e^{j \omega T}\right) X\left(e^{j \omega T}\right)+\sum_{m=1}^{M-1} V_{m}\left(e^{j \omega T}\right) X\left(e^{j(\omega T-2 \pi m / M)}\right) .
$$

Here,

$$
V_{0}\left(e^{j \omega T}\right)=\frac{1}{M} \sum_{n=0}^{M-1} \bar{A}_{n}\left(e^{j \omega T}\right)
$$

and

$$
V_{m}\left(e^{j \omega T}\right)=\frac{1}{M} \sum_{n=0}^{M-1} e^{-j 2 \pi m n / M} \bar{A}_{n}\left(e^{j(\omega T-2 \pi m / M)}\right) .
$$

In (2.23) and (2.24), $\bar{A}_{n}\left(e^{j \omega T}\right), n \in[0,1, \ldots, M-1]$, is the $2 \pi$-periodic extension of $A_{n}(j \omega)$ in (2.11). It can be noted from (2.22) that with $V_{0}\left(e^{j \omega T}\right)=1$ and $V_{m}\left(e^{j \omega T}\right)=0, m=1,2, \ldots, M-1$, we attain perfect reconstruction. However, to achieve a specified SFDR, it is sufficient to ensure that the distortion and aliasing functions approximate unity and zero, respectively, within the band $\omega T \in\left[-\omega_{\mathrm{c}} T, \omega_{\mathrm{c}} T\right]$. Thus, the filter coefficients $h_{n}(k)$ can be determined such that for a maximum specified reconstruction error $\delta$,

$$
\left|V_{m}\left(e^{j \omega T}\right)-a_{m}\right| \leq \delta_{m}, \omega T \in\left[-\omega_{\mathrm{c}} T+\frac{2 \pi m}{M}, \omega_{\mathrm{c}} T+\frac{2 \pi m}{M}\right] .
$$

with $\delta_{m}<\delta$ for $m=1,2, \ldots, M-1$. Typically, the requirements on the distortion error $\delta_{0}$ is not the same as the errors in the aliasing terms, $\delta_{m}$, $m=1,2, \ldots, M-1$. In (2.25), $a_{0}=1$ and $a_{m}=0$ for $m=1,2, \ldots, M-1$. Unlike the least-squares design which can be carried out online, the minimax approach is more suitable for offline design.

\subsection{Reconstructor Complexity}

In the literature, the complexity of a reconstructor is often measured in terms of the number and type of multipliers required to implement the reconstructor $[57,63]$. For example, variable-coefficient multipliers are required if the 
filter coefficients in the reconstructor have to be redetermined whenever the mismatch parameters change. In such cases, an additional online redesign block is required to redetermine the coefficients, resulting in increased chip area and power consumption. As can be seen from (2.21), in an $M$-channel TI-ADC, online redesign of the time-varying FIR reconstructor would require $M-1$ separate $(N+1) \times(N+1)$ matrix inversions. Thus, it is still important to have a simpler online redesign block.

Unlike the online redesign block, the reconstructor always runs at the output of the TI-ADC. Hence, a rough measure of the overall power consumption is obtained from the number of arithmetic operations per corrected output sample (computational complexity) as well as the number of delay elements in the reconstructor. In regular time-varying FIR reconstructors [57], the number of adders and delay elements scale proportionally with the number of multipliers. Hence, the computational complexity of the reconstructor is measured in terms of the number of multiplications per corrected output sample. It can be noted from (2.9) that, in the time-varying FIR reconstructor, each corrected output sample requires $N+1$ multiplications where the value of $N$ depends on the magnitude of the mismatch errors, the bandwidth supported by the reconstructor, and how small the reconstruction error should be. Further, in reconstructor implementations, variable-coefficient multipliers are used for filter coefficients whose values are redetermined online. However, compared to variable-coefficient multipliers, efficient techniques can be used to implement fixed-coefficient multipliers $[64,65]$. Thus, in order to reduce the area and power consumed by the reconstructor, it is desirable to have a reconstruction scheme with as few variable-coefficient multipliers as possible.

\subsection{Low-Complexity Reconstruction Schemes}

In the papers included in Part II of this thesis, we consider the reconstruction of uniform-grid samples from the nonuniformly sampled signal at the output of TI-ADCs. There, we assume that the channel ADCs have only static time-skew errors. Among the reconstructors used in such TI-ADCs, the regular time-varying FIR reconstructor [57] requires the minimal number of multiplications per corrected output sample. However, all the coefficients in the regular reconstructor have to be redetermined online when the timeskew errors change. Hence, all the filter coefficients in this reconstructor are 
implemented using expensive variable-coefficient multipliers. At the other end of the complexity spectrum is the reconstructor which makes use of differentiator-multiplier cascade (DMC) [18]. For a given specification, the DMC reconstructor requires the least number of variable-coefficient multipliers. ${ }^{3}$ Also, the DMC reconstructor does not require an online redesign block as it can be reconfigured by directly updating the coefficients of the variable multipliers with the newly estimated time-skew errors. However, due to a cascaded structure which does not allow the sharing of delay elements, the DMC reconstructor requires more delay elements as well as longer delays compared to the regular reconstructor. In Part II, we propose three reconstruction schemes that offer trade-offs between online redesign and reconstructor complexities. To reduce the complexity, these reconstructors utilize a two-rate based approach [66] which earlier has been used only for uniformly sampled signals [67-75]. However, here, the two-rate based approach is extended for the reconstruction of nonuniformly sampled signals which requires new design techniques.

\subsubsection{Two-Rate Based Approach}

The two-rate based approach, on a principle level, is shown in Fig. 2.7(b). Here, the input is first interpolated by a factor of two by using an upsampler and a half-band filter $F(z)$. The interpolated nonuniformly sampled signal is then reconstructed using the reconstructor $G_{n}(z)$. Finally, a downsampler is used to make the output rate equal to that of the input. In practice, the two-rate based structure in Fig. 2.7(b) is implemented using an equivalent single-rate structure as explained in the papers of Part II. Compared to the regular FIR reconstructor in Fig. 2.7(a), the single-rate structure achieves lower complexity. This is because the majority of the multipliers can now be implemented using fixed-coefficient multipliers whereas, due to the interpolation, $G_{n}(z)$ requires only a low order reconstructor and hence, fewer variable-coefficient multipliers.

The principle behind the reduction in the complexity of the filter $G_{n}(z)$ is illustrated in Fig. 2.8 which plots the variation of the reconstructor order with its bandwidth $\omega_{\mathrm{c}} T$. In Fig. 2.8, the reconstructor is required to recover the uniform-grid samples from the output of a four-channel TI-ADC with

\footnotetext{
${ }^{3}$ A special case is for $M=2$ where the structure in [59] is the more efficient than the DMC reconstructor.
} 
(a)

$$
v(n) \rightarrow H_{n}(z) \rightarrow \tilde{x}(n)
$$

(b)

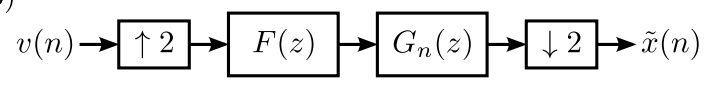

Figure 2.7: (a) Regular time-varying FIR reconstructor. (b) Two-rate based reconstructor.

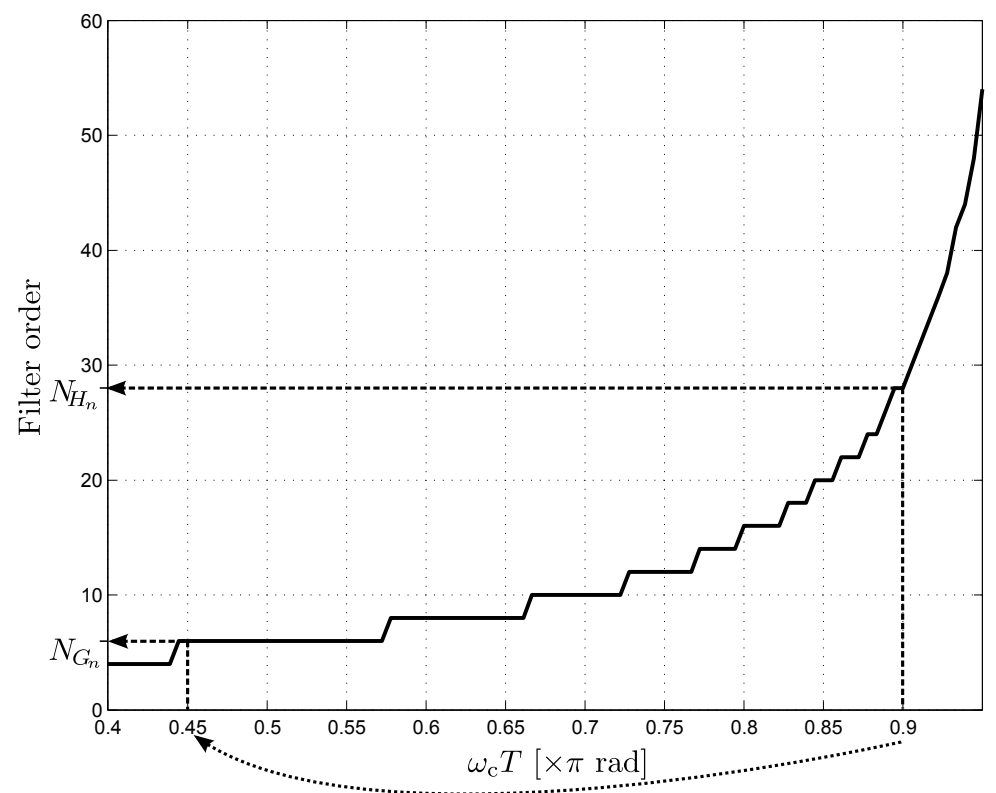

Figure 2.8: Filter order versus reconstructor bandwidth for $H_{n}(z)$ in a fourchannel TI-ADC with time-skew errors $\varepsilon_{0,1,2,3}=[-0.02,0.02,-0.02,0.02]$ and with reconstruction error $P_{n} \leq-80 \mathrm{~dB}$. 
channel time-skew errors $\varepsilon_{0,1,2,3}=[-0.02,0.02,-0.02,0.02]^{4}$ such that, after reconstruction, the error $P_{n}$ in $(2.14)$ is below $-80 \mathrm{~dB}$. It can be seen that as the bandwidth $\omega_{\mathrm{c}} T$ increases, the order of the reconstructor increases roughly by $K\left(\pi-\omega_{\mathrm{c}} T\right)^{-1}$ where $K$ is a constant. As illustrated, if the bandwidth of the reconstructor is $\omega_{\mathrm{c}} T=0.9 \pi$, the regular time-varying FIR reconstructor $H_{n}(z)$ would require a filter order of $N_{H_{n}}=28$. However, due to the interpolation in the two-rate based approach shown in 2.7(b), the bandwidth to be supported by the $G_{n}(z)$ reconstructor is reduced to $0.45 \pi$. Hence, the order required for $G_{n}(z)$ is lower than that of $H_{n}(z)$. Also, since the filter $F(z)$ is a half-band FIR filter, its every other impulse response coefficient is equal to zero [32]. Further, since $F(z)$ is a linear-phase FIR filter, the nonzero impulse response coefficients are symmetric, and hence, can be implemented with half the number of multipliers.

In the proposed reconstructors in Part II, $F(z)$ is designed offline and its coefficients are fixed. The coefficients of $F(z)$ are determined such that they can be used for all $\varepsilon_{n} \in\left[-\varepsilon_{\max }, \varepsilon_{\max }\right]$. Even though the design is carried out offline, it can still be time consuming with design times ranging from several minutes to hours, especially for small reconstruction errors, wider bandwidths and/or for larger $M$. However, at the cost of a marginal increase in the filter order of $G_{n}(z)$, the offline design of $F(z)$ can be simplified by using a standard half-band filter that can be designed straightforwardly.

In Paper A of Part II, we propose a two-rate based reconstructor for twochannel TI-ADCs which is a popular TI-ADC configuration [76-79]. The basic two-rate based approach in Paper A is extended to a general $M$-channel TI-ADC reconstruction scheme in Paper B. Compared to the regular reconstructor, the reconstructor in Paper B requires fewer variable-coefficient multipliers and simpler online redesign block. Though the DMC reconstructor [18] requires fewer variable multipliers as well as no online redesign, the reconstructor in Paper B requires significantly fewer delay elements which also needs to be taken into account while comparing complexities especially in low-power applications like application-specific integrated circuits (ASICs).

Finally, in Paper C, we use the two-rate based approach to extend the multivariate impulse response reconstructor, originally proposed in [80]. This reconstruction scheme is attractive for $M$-channel TI-ADCs with a small

\footnotetext{
${ }^{4}$ It is noted that, in practice, for TI-ADC implementations, the time-skew error of the reference channel is assumed to be zero.
} 
maximum possible time-skew error $\varepsilon_{\max }$. Unlike the reconstruction scheme in Paper B, the scheme in Paper $\mathrm{C}$ requires no online redesign block. However, for a specific design example, it was seen that this reconstructor required more operations per corrected output sample than the other reconstructors.

\subsection{Summary}

In this chapter we reviewed the effect of channel mismatch errors in TIADCs. It was shown that due to mismatches between the channel ADCs, aliasing terms appear at the TI-ADC output which degrades the achievable resolution. We reviewed two different errors metrics and the corresponding design for time-varying FIR filters. Further, we discussed that the complexity of the reconstructor can be measured in terms of the number of operations per corrected output sample, the number of fixed and variable-coefficient multipliers that are required to implement the reconstructor, the number of delay elements, and the complexity involved in the online redesign of the reconstructor. The existing reconstruction scheme that gives minimal computational complexity, overall delay, and number of delay elements has high redesign complexity. On the other hand, the scheme which has no redesign complexity requires more arithmetic operations and higher overall delay and number of delay elements. Finally, for correcting static time-skew errors in TI-ADCs, we propose three digital reconstructors which allows the designer to make trade-offs between online redesign and reconstructor complexities. 


\section{Chapter 3}

\section{Sub-Nyquist Sampling of Sparse Multi-Band Signals}

It is well known that uniform sampling of a signal which is bandlimited to $f<f_{0}$, at a sampling frequency of $f_{s} \geq 2 f_{0}$, results in a uniformly spaced sequence of samples that can be used to reconstruct the original signal. However, in many cases, the signal is sparse in a sense that the actual information is contained in a bandwidth much less than $f_{0}$. One example is a frequency-hopping communication system where there are one or more narrowband carriers (active subbands) that change their center frequencies within the band $\left[0, f_{0}\right)$ at a certain switching rate. In other words, such signals are locally narrowband (in a time frame) but globally wideband (over several time frames). In such cases, the traditional approach would require a high-speed ADC operating at a rate of $f_{s} \geq 2 f_{0}$. Hence, within a time frame, the signal is heavily oversampled and the ADC will unnecessarily consume a substantial amount of power.

Sub-Nyquist sampling is becoming increasingly popular in wideband communication systems, especially in battery-powered applications where highspeed uniform sampling results in higher power consumption. In such sampling schemes, the average sampling rate can be much lower than $2 f_{0}$ but still large enough to capture the information content in the signal. In this chapter, we focus on the multi-band (or multi-coset) sampling approach where the use of cyclic nonuniform sampling (CNUS) helps to reduce the average sampling rate to (in principle) the Landau minimal sampling rate which is determined by the frequency occupancy [25]. It is known that, 


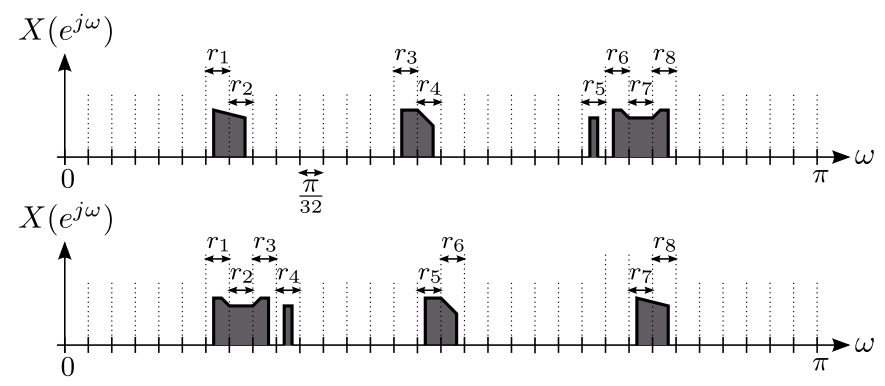

Figure 3.1: Spectrum of a sparse multi-band signal with $M=32$ and four active users occupying $K=8$ active granularity bands. The top and bottom plots show the occupied granularity bands at two different time frames.

given the sampling pattern for the CNUS approach, the reconstruction can be carried out via a set of ideal multi-level synthesis filters [81]. However, the straightforward CNUS reconstruction filters have very high design and reconstructor complexity. Also, in spread-spectrum communication systems where the active subband locations are different for different time frames, the reconstruction scheme should support online reconfigurability without increasing the complexity.

\subsection{Sub-Nyquist Cyclic Nonuniform Sampling}

Assume that $x_{a}(t)$ is a real-valued continuous-time signal that carries information within the frequency band $\omega \in\left(-2 \pi f_{0}, 2 \pi f_{0}\right), f_{0}<1 /(2 T)$. Uniform sampling of $x_{a}(t)$ at a sampling frequency of $f_{s}=1 / T$ results in a discrete-time sequence $x(n)=x_{a}(n T)$. For the sake of simplicity, hereafter we assume that $T=1$. Now it is assumed that the band $\omega \in[0, \pi]$ is divided into $M$ granularity bands of equal width $\pi / M$. In sparse multi-band signals, at any given time frame, only $K$ of the $M$ granularity bands $(K<M)$ are allocated to users. Here, we use $r_{i} \in[0,1, \ldots, M-1], i=1,2, \ldots, K$ to denote the active granularity bands assigned to users. A user can occupy one or several consecutive granularity bands. Further, to be able to design practical filters, we assume a certain amount of redundancy (oversampling) which corresponds to transition bands between user bands. Figure 3.1 shows the principle spectrum of a sparse multi-band signal when $M=32$ and $K=8$. The top plot in Fig. 3.1 corresponds to the scenario where the active granularity bands are $r_{1,2,3,4,5,6,7,8}=[6,7,14,15,22,23,24,25]$ whereas 
(a)

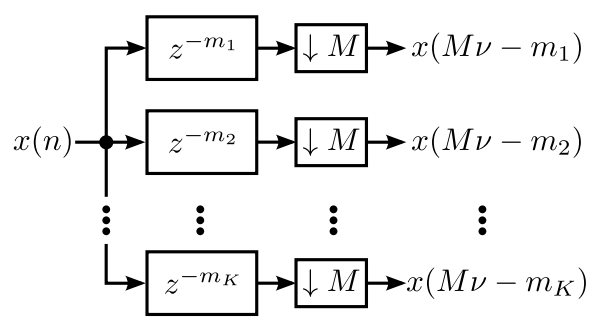

(b)

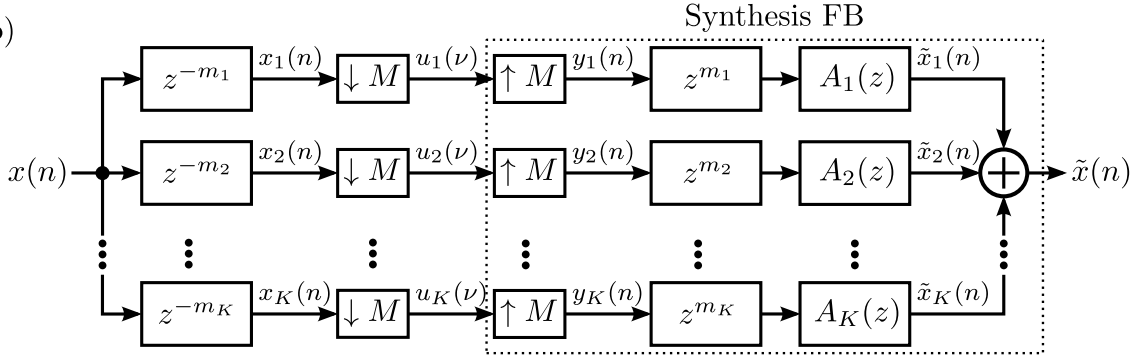

Figure 3.2: (a) Equivalent representation of the available samples $u_{\ell}(\nu)=$ $x\left(M \nu-m_{\ell}\right), \ell=1,2, \ldots, K$, when the input $x(n)$ is obtained via sub-Nyquist CNUS. (b) Reconstruction using multi-level synthesis filters [81].

the bottom plot corresponds to active granularity bands $r_{1,2,3,4,5,6,7,8}=$ $[6,7,8,9,15,16,24,25]$.

In the case of such sparse multi-band signals, uniform sampling will generate more samples than what is required to prevent information loss. The number of samples that is generated during the sampling process can be reduced by using CNUS which only uses a subset $x\left(M n-m_{\ell}\right), \ell=1,2, \ldots, K$ with $m_{\ell} \in[0,1, \ldots, M-1]$ of the uniform samples $x(n)$. The available samples $u_{\ell}(\nu)=x\left(M \nu-m_{\ell}\right)$ can be considered as obtained from the uniform-grid samples $x(n)$ as shown in Fig. 3.2(a). A practical implementation of the CNUS is an $M$-channel TI-ADC where only a subset of the channels are used. By properly selecting the sampling instants $m_{\ell}$ [82-84], a reconstructor can be used to recover the uniformly sampled sequence $x(n)$ from $x\left(M n-m_{\ell}\right)$ for a given set of $K$ granularity bands. 


\subsection{Reconstruction Using Multi-Level Synthesis Filters}

A reconstruction scheme using a set of $K$ multi-level synthesis filters $A_{\ell}(z)$, $\ell=1,2, \ldots, K$, as shown in Fig. 3.2(b), was proposed in [81]. Below, we show that using ideal multi-level synthesis filters $A_{\ell}(z)$ in Fig. 3.2(b) we can, in principle, perfectly recover $x(n)$ from the available samples $u_{\ell}(\nu)$ [81].

To show this, we start with the $z$-transforms of the sequences $x_{\ell}(n), \ell=$ $1,2, \ldots, K$ in Fig. 3.2(b) which can be expressed as

$$
X_{\ell}(z)=z^{-m_{\ell}} X(z) \text {. }
$$

Since the sequences $u_{\ell}(\nu)$ in Fig. 3.2(b) are obtained by decimating $x_{\ell}(n)$, their $z$-transforms can be written as

$$
U_{\ell}(z)=\frac{1}{M} \sum_{q=0}^{M-1} X_{\ell}\left(z^{1 / M} e^{-j 2 \pi q / M}\right) .
$$

Using (3.1) and (3.2), the $z$-transforms of $y_{\ell}(n)$ can be written as

$$
\begin{aligned}
Y_{\ell}(z) & =U_{\ell}\left(z^{M}\right)=\frac{1}{M} \sum_{q=0}^{M-1} X_{\ell}\left(z e^{-j 2 \pi q / M}\right) \\
& =\frac{1}{M} \sum_{q=0}^{M-1} z^{-m_{\ell}} e^{j 2 \pi q m_{\ell} / M} X\left(z e^{-j 2 \pi q / M}\right) .
\end{aligned}
$$

Now, the $z$-transforms of $\tilde{x}_{\ell}(n)$ are given by

$$
\widetilde{X}_{\ell}(z)=z^{m_{\ell}} A_{\ell}(z) Y_{\ell}(z)=\frac{1}{M} A_{\ell}(z) \sum_{q=0}^{M-1} e^{j 2 \pi q m_{\ell} / M} X\left(z e^{-j 2 \pi q / M}\right) .
$$

Then, the Fourier transform of the output $\widetilde{X}(z)$ can be written as

$$
\widetilde{X}(z)=\sum_{\ell=1}^{K} \widetilde{X}_{\ell}(z)=\frac{1}{M} E_{0}(z) X(z)+\frac{1}{M} \sum_{q=1}^{M-1} E_{q}(z) X\left(z e^{-j 2 \pi q / M}\right)
$$

where

$$
E_{q}(z)=\frac{1}{M} \sum_{\ell=1}^{K} e^{j 2 \pi q m_{\ell} / M} A_{\ell}(z)
$$


for $q=0,1, \ldots, M-1$. In (3.5), the terms $X\left(z e^{-j 2 \pi q / M}\right), q=1,2, \ldots, M-1$ correspond to the $M-1$ aliasing terms of $X(z)$. Since, for perfect reconstruction, $\widetilde{X}(z)=X(z)$, it follows from (3.5) that we must have $E_{0}(z)=M$ and $E_{q}(z)=0$ for $q=1,2, \ldots, M-1$. Now, assuming that the magnitude responses of the ideal synthesis filters $A_{\ell}(z)$ are zero in the $M-K$ nonactive subbands, the filters $A_{\ell}(z)$ are to be designed such that they cancel the aliasing terms that fall into the $K$ active subbands. Since the Nyquist band is divided into $M$ non-overlapping subbands, the signal in each active subband $|\omega| \in\left[r_{k} \pi / M,\left(r_{k}+1\right) \pi / M\right], r_{k} \in[0,1, \ldots, M-1]$, fulfills the sampling theorem for bandpass decimation. Hence, the shifted copies of an active subband will not overlap with itself. Further, from each of the remaining $K-1$ active bands, exactly one shifted copy will also fall into the above active subband. Due to this, in any active subband, $K-1$ aliasing terms will be present. Thus, the frequency response of the $K$ synthesis filters $A_{\ell}\left(e^{j \omega}\right)$ in the active subband $\omega \in\left[r_{k} \pi / M,\left(r_{k}+1\right) \pi / M\right]$ can be determined by solving the systems of $K$ equations

$$
\underbrace{\left[\begin{array}{cccc}
1 & 1 & \cdots & 1 \\
e^{j \frac{2 \pi q_{1}^{(k)} m_{1}}{M}} & e^{j \frac{2 \pi q_{1}^{(k)} m_{2}}{M}} & \cdots & e^{j \frac{2 \pi q_{1}^{(k)} m_{K}}{M}} \\
\vdots & \vdots & \ddots & \vdots \\
e^{j \frac{2 \pi q_{K}^{(k)} m_{1}}{M}} & e^{j \frac{2 \pi q_{K}^{(k)} m_{2}}{M}} & \cdots & e^{j \frac{2 \pi q_{K}^{(k)} m_{K}}{M}}
\end{array}\right]}_{\mathbf{E}^{(k)}}\left[\begin{array}{c}
A_{1}\left(e^{j \omega}\right) \\
A_{2}\left(e^{j \omega}\right) \\
\vdots \\
A_{K}\left(e^{j \omega}\right)
\end{array}\right]=\left[\begin{array}{c}
M \\
0 \\
\vdots \\
0
\end{array}\right]
$$

where $q_{i}^{(k)} \in[1,2, \ldots, M-1], i=1,2, \ldots, K$, correspond to the $2 \pi q_{i}^{(k)} / M$ shifted copies (aliasing terms) of $X(z)$ that fall into the $k$ th subband. A solution for (3.7) exists if the matrix $\mathbf{E}^{(k)}$ is invertible. For a given set of active subband locations, this can be ensured through a proper selection of the sampling instants $m_{\ell}[81-84]$.

Next, we illustrate the design of the multi-level synthesis filters using an example. Here, assume that $M=8, K=3$, and the active subbands bands are $r_{1,2,3}=\{1,4,6\}$. That is, the spectrum of the uniformly sampled signal $X\left(e^{j \omega}\right)$ is as shown in Fig. 3.3. For the sampling points $m_{1,2,3}=\{0,3,5\}$, we will have three synthesis filters $A_{1}(z), A_{2}(z)$, and $A_{3}(z)$. In the following, for simplicity, we assume that the reconstruction is performed using ideal synthesis filters. As can be seen from Fig. 3.3, the aliasing terms $q_{1,2}^{(1)}=$ $\{3,4\} q_{1,2}^{(2)}=\{3,7\}$, and $q_{1,2}^{(3)}=\{1,4\}$ fall into the subbands $r_{1}, r_{2}$, and $r_{3}$, 


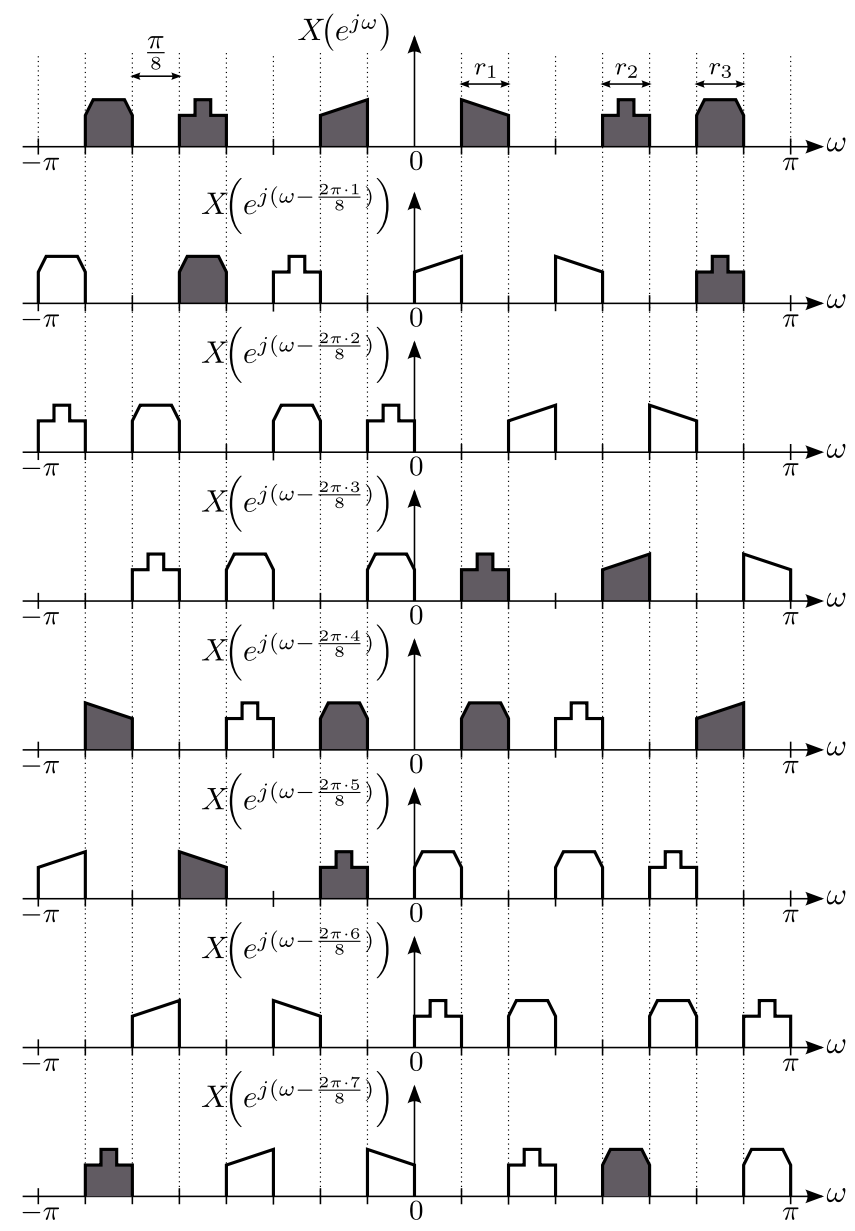

Figure 3.3: Spectrum of a uniformly sampled sparse multi-band signal and its aliasing terms. Here, $M=8$ and $K=3$. The shaded terms in $X\left(e^{j(\omega-2 \pi q / M)}\right), q=1,2, \ldots, 7$, are the aliasing terms that fall in the active subbands. 
respectively. Thus, in the subband $r_{1},(3.7)$ becomes

$$
\left[\begin{array}{ccc}
1 & 1 & 1 \\
e^{j \frac{2 \pi 3 \cdot 0}{8}} & e^{j \frac{2 \pi 3 \cdot 3}{8}} & e^{j \frac{2 \pi 3 \cdot 5}{8}} \\
e^{j \frac{2 \pi \cdot 0}{8}} & e^{j \frac{2 \pi 4 \cdot 3}{8}} & e^{j \frac{2 \pi 4 \cdot 5}{8}}
\end{array}\right]\left[\begin{array}{l}
A_{1}\left(e^{j \omega}\right) \\
A_{2}\left(e^{j \omega}\right) \\
A_{3}\left(e^{j \omega}\right)
\end{array}\right]=\left[\begin{array}{c}
M \\
0 \\
0
\end{array}\right] .
$$

Solving (3.8), we get the response of synthesis filters in subband $r_{1}$ as $A_{1}\left(e^{j \omega}\right)=4, A_{2}\left(e^{j \omega}\right)=5.2263 e^{-j 1.1781}$, and $A_{3}\left(e^{j \omega}\right)=5.2263 e^{j 1.1781}$. Similarly, we get $A_{1}\left(e^{j \omega}\right)=0, A_{2}\left(e^{j \omega}\right)=5.6569 e^{-j 0.7854}$, and $A_{3}\left(e^{j \omega}\right)=$ $5.6569 e^{j 0.7854}$ in subband $r_{2}$, and $A_{1}\left(e^{j \omega}\right)=4, A_{2}\left(e^{j \omega}\right)=2.1648 e^{-j 0.3927}$, and $A_{3}\left(e^{j \omega}\right)=2.1648 e^{j 0.3927}$. The responses of $A_{1}\left(e^{j \omega}\right), A_{2}\left(e^{j \omega}\right)$, and $A_{2}\left(e^{j \omega}\right)$ in the negative frequency bands are obtained by taking the complex conjugate of the responses of $A_{1}\left(e^{j \omega}\right), A_{2}\left(e^{j \omega}\right)$, and $A_{2}\left(e^{j \omega}\right)$, respectively, in the corresponding positive frequency bands.

\subsubsection{Complexity}

In practice, the coefficients of the synthesis filters can be determined straightforwardly, assuming no a priori relations between the filters. If $N$ is the order of $A_{\ell}(z)$, then with polyphase implementation, the reconstructor has a computational complexity of roughly $N K / M$ multiplications per corrected output sample. As $M$ increases, $K$ also increases such that the ratio $K / M$ remains the same. However, as $M$ increases, the subbands become narrower and as a result, $N$ increases. Due to this, the reconstructor can become intolerably costly in real-time applications. Also, all the synthesis filters have to be be redesigned if the location of the active subbands change at a later time frame. Since regular filter design is cumbersome to be carried out online when the filter order is high, the online design complexity of the reconstructor is high.

\subsection{Reconstruction Using Analysis and Synthesis Filters}

In Paper D of Part III, we derive an efficient reconstruction scheme, shown here in Fig. 3.4, by describing the reconstruction in terms of both analysis and synthesis filters as shown in Fig. 3.5. Each analysis filter $B_{k}(z)$ extracts one of the $K$ active granularity bands. The filtering by $B_{k}(z)$ is followed by 


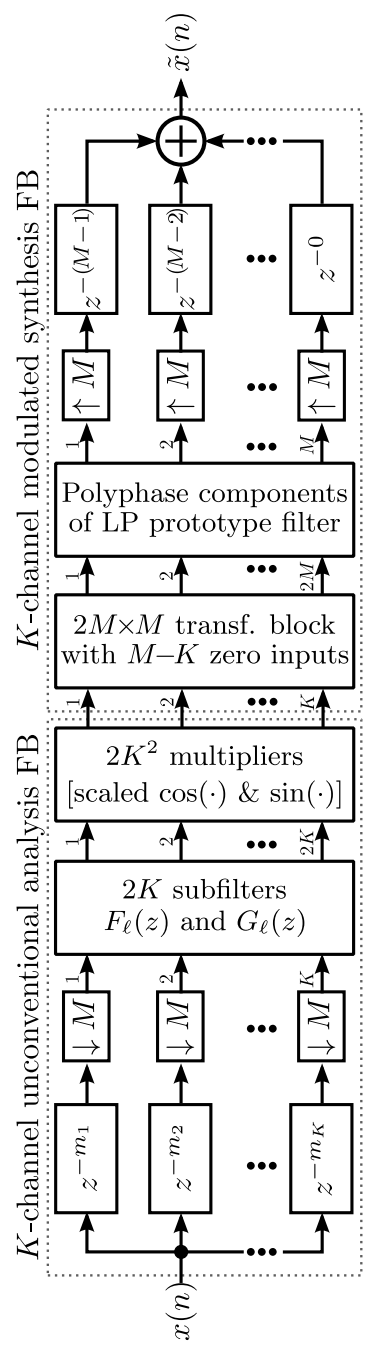

Figure 3.4: Efficient reconfigurable reconstructor proposed in Paper D. 


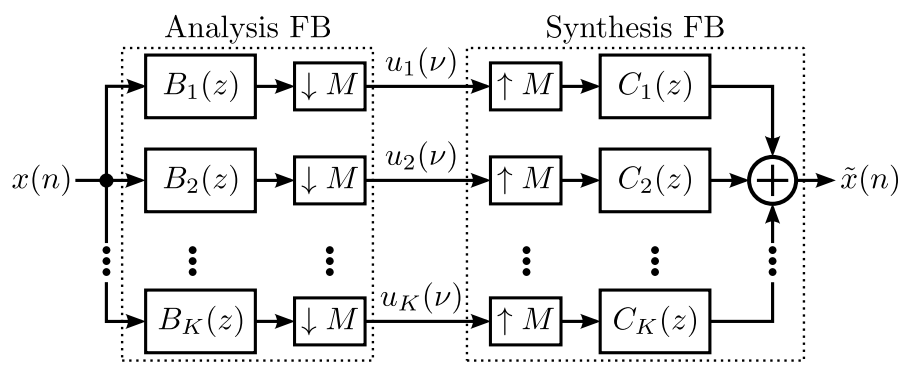

Figure 3.5: Reconstruction of sub-Nyquist sampled sparse multi-band signal using analysis and synthesis filters.

downsampling by $M$ so as to have the extracted active granularity band at the lower sampling rate $f_{s} / M$. The low-rate signal is then placed at the original active granularity band location at the higher rate $f_{s}$ via upsampling by $M$ followed by bandpass filtering via $C_{k}(z)$. The synthesis filters $C_{k}(z)$ thus provide a bank of $K$ different conventional bandpass filters which can be implemented using an efficient cosine-modulated FB consisting of a prototype filter and a transform block (see Section 1.1.8). Each analysis filter $B_{k}(z)$ is an unconventional bandpass decimation filter with $K$ non-zero polyphase branches. In Paper D, we show that the multi-level synthesis filters in [81] can be expressed in terms of analysis and synthesis filters as shown in Fig. 3.5. However, to get further insights and understanding of the efficient reconfigurable reconstruction scheme described in Paper D, we derive the scheme in Fig. 3.5 using a different approach in Appendix A.

\subsubsection{Complexity}

In Paper D, we show that expressing the reconstruction problem in terms of analysis and synthesis FB as shown in Fig. 3.5, allows us to derive the efficient reconfigurable reconstructor shown in Fig. 3.4. There, we show that compared to the reconstructor that uses only a synthesis FB [81], the proposed reconstructor can deliver an order-of-magnitude reduction in computational complexity. In addition to this, the proposed reconstructor can be reconfigured online with a single $K \times K$ matrix inversion. Table 1 illustrates the complexity savings of the proposed reconstructor when the information containing frequency bands are $\{[3.2-3.8],[7.2-7.8],[11.2-12.8]\} \times \pi / 16$, $M=128, K=18$, and the reconstructor is designed to keep the aliasing terms below $-40 \mathrm{~dB}$ (for details, see Example 2 in Section 7 of Paper D). 
Table 1: Reconstructor complexity comparison.

\begin{tabular}{|c|c|c|c|}
\hline Reconstructor & \multicolumn{3}{|c|}{ Complexity $^{5}$} \\
& $\mathcal{C}$ & $\mathcal{N}$ & Reconfiguration \\
\hline \hline Only synthesis FB [81] & 164 & 20934 & $18[1163 \times 1163]$ \\
\hline Proposed & 24 & 648 & One $[18 \times 18]$ \\
\hline
\end{tabular}

\subsection{Summary and Future Extension}

In this chapter we reviewed the concept of using CNUS for the sub-Nyquist sampling of sparse multi-band signals. It was shown that a TI-ADC with only few active channel ADCs can be used to straightforwardly implement the sub-Nyquist CNUS scheme. We also reviewed the existing reconstruction scheme which uses only a synthesis FB. Here, we introduced a reconfigurable reconstruction scheme that offers substantial reduction in complexity. The proposed reconstruction scheme is derived by describing the reconstruction in terms of both the analysis and synthesis FB. Since the polyphase components of the filters in the analysis FB are generalized FD filters, all the analysis filters are expressed using a common set of fixed subfilters which are designed offline. The different analysis filters are then realized using different sets of multipliers. When the reconstructor is reconfigured online, only the coefficients of these multipliers have to be redetermined, thus lowering the complexity of the online redesign block.

In our discussions, we assumed that the channel ADCs have no mismatch errors. As seen in Chapter 2, the achievable resolution of a TI-ADC can be degraded by channel mismatch errors. Using an approach similar to the sub-band based reconstructor in Section 4.2.2 of Chapter 4, the proposed reconstruction scheme can be modified to compensate for static time-skew errors in the active channel ADCs. As in the case of the sub-band based reconstructor, here also the reconstructor can be reconfigured easily whenever the time-skew errors change. However, the above scheme cannot be straightforwardly used for correcting frequency-response mismatches which can be a limiting factor in applications requiring very high sampling rates. Thus, an interesting future extension is to derive a structure that can be used to correct frequency-dependent mismatches as well.

\footnotetext{
${ }^{5} \mathcal{C}$ and $\mathcal{N}$ represent the number of multiplications per corrected output sample and the number of multipliers to be updated during reconfiguration, respectively. The reconfiguration complexity is the number of online matrix inversions.
} 


\section{Chapter 4}

\section{Reconstruction in TI-ADCs with Missing Samples}

\subsection{TI-ADCs with Missing Samples}

In Chapter 2, we showed that due to mismatches between the channel ADCs, the output of a conventional TI-ADC will be a nonuniformly sampled version of the bandlimited input signal $x_{a}(t)$. In particular, it was shown that timeskew mismatches between the channel ADCs result in a nonunifom-sampling sequence $v(n)$ given by

$$
v(n)=x_{a}\left(n T+\varepsilon_{n} T\right)
$$

where $T$ is the sampling period and $\varepsilon_{n} T$ represents, for the $n$th sample, the deviation of the actual sampling instant from the uniform sampling instant $n T$. In the rest of this chapter, we assume $T=1$ for simplicity. It was also shown in Chapter 2 that digital reconstructors can be used at the output of TI-ADCs to compensate for these mismatch errors and approximately recover the uniformly sampled signal $x(n)$. In practice, the mismatch parameters have to be estimated first before they can be used by the reconstructor. An iterative background estimation scheme that achieves robust estimation compared to other background estimation schemes was proposed in [26]. In this scheme, a known calibration signal is injected to the TI-ADC input at predefined sampling instants $t=r M_{c}, r \in \mathbb{Z}$, as shown in Fig. 4.1. Thus, every $M_{c}$ th output sample from the TI-ADC corresponds to a nonuniformly 


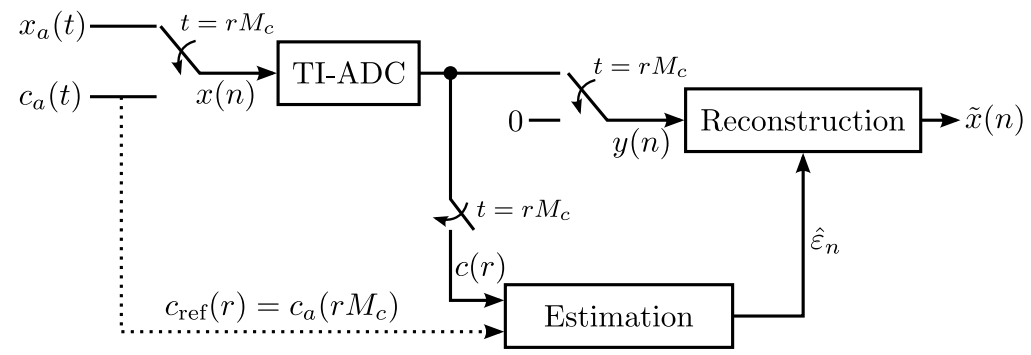

Figure 4.1: Block digram of the background estimation scheme in [26] where a known calibration signal is used to estimate the mismatch parameters.

sampled version of $c_{a}(t)$. The nonuniformly sampled calibration sequence $c(r)$ is fed to an estimator which compares $c(r)$ with a known reference sequence $c_{\text {ref }}(r)=c_{a}\left(r M_{c}\right)$, and thereby estimates the mismatch between the channel ADCs. In order to ensure that $c(r)$ is composed of samples from all the channel ADCs, in an $M$-channel TI-ADC, $M_{c}$ is chosen such that $M$ and $M_{c}$ are co-prime. At the input sampling instants reserved for the calibration signal, the input signal $x_{a}(t)$ will not be sampled by the TI-ADC resulting in missing samples at the TI-ADC output. Thus, the input to the reconstructor, $y(n)$, is given by

$$
y(n)= \begin{cases}0, & n=r M_{c} \\ v(n), & \text { otherwise. }\end{cases}
$$

Figure 4.2 illustrates this for a three-channel TI-ADC $(M=3)$ with timeskew errors where every fourth sampling instant $\left(M_{c}=4\right)$ is reserved for the calibration signal. Thus, the reconstructor used in such TI-ADCs should recover these missing samples in addition to compensating for the mismatch errors.

\subsection{Reconstruction Schemes}

In [26], the reconstructor was realized using an iterative scheme with good convergence rate. However, this scheme has a high computational complexity which is measured in terms of the number of operations required per corrected output sample. Further, the reconstructor makes use of a recursive structure which, in addition to limiting the maximum data rate [85], can also lead to stability problems. In this section, we provide an overview of the three nonrecursive FIR reconstructors proposed in Part IV. 
(a)

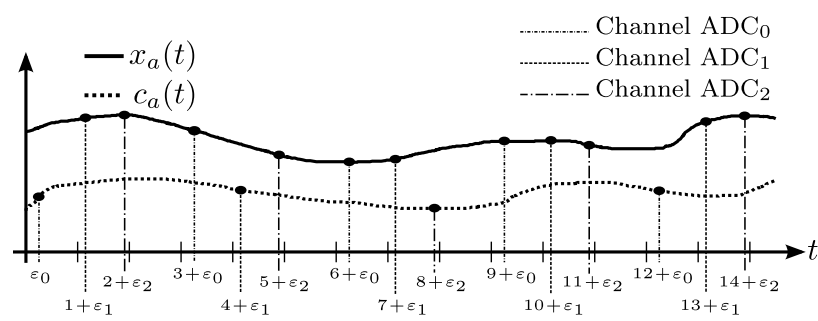

(b)

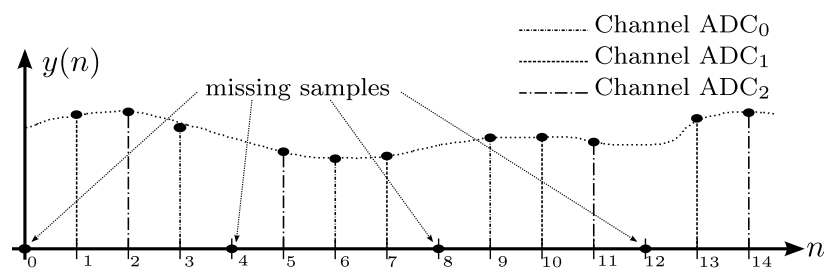

Figure 4.2: (a) Illustration of the sampling instants in a three-channel TI$\operatorname{ADC}(M=3)$ where every fourth sampling instant $\left(M_{c}=4\right)$ is reserved for the calibration signal. (b) Corresponding nonuniformly sampled sequence at the output of the three-channel TI-ADC.

\subsubsection{Constrained Time-Varying FIR Reconstructor}

As seen from Section 2.1.1, due to mismatches, the output of a TI-ADC without missing samples is an $M$-periodically nonuniformly sampled version of the input $x_{a}(t)$. In Section 2.2, it was shown that the uniform-grid samples can be recovered from the $M$-periodically nonuniformly sampled signal using an $M$-periodically time-varying FIR reconstructor, $h_{n}(k)$. Here, due to the missing samples, the TI-ADC output $y(n)$ is an $M M_{c}$-periodically nonuniformly sampled version of $x_{a}(t)$. That is, $h_{n}(k)=h_{n+M_{\mathrm{t}}}(k)$ where $M_{\mathrm{t}}=M M_{c}$. Hence, we could use an $M_{\mathrm{t}}$-periodically time-varying FIR reconstructor to recover the uniform-grid samples from $y(n)$. However, unlike the time-varying reconstructor in [57], here, the missing samples in $y(n)$ restrict some of the impulse response coefficients to be zero. Specifically,

$$
h_{n}(k)=0, \quad \forall k=-n+r M_{c}, r \in \mathbb{Z} .
$$

Assuming that, for a given $n, R_{n}$ denotes the number of non-zero impulse response coefficients in $h_{n}(k)$, the reconstructed output $\tilde{x}(n)$ can be written as

$$
\widetilde{x}(n)=\sum_{i=1}^{R_{n}} y\left(n-k_{i}\right) h_{n}\left(k_{i}\right)
$$


where $k_{i} \in\left[-N_{h_{n}} / 2,-N_{h_{n}} / 2+1, \ldots, N_{h_{n}} / 2\right], i=1,2, \ldots, R$, represent the indices of the non-zero impulse response coefficients in $h_{n}(k)$. In (4.4), for simplicity, it is assumed that $h_{n}(k)$ is a noncausal filter (see Footnote 2 ) with even order $N_{h_{n}}$.

It was seen in Section 2.2 that in the case of the time-varying reconstructor, how well $\tilde{x}(n)$ approximates the uniform-grid samples $x(n)$ depends on the approximation error between $A_{n}(j \omega)$ in (2.11) and unity. This is also applicable for the constrained time-varying reconstructor except, here, due to the missing samples, $A_{n}(j \omega)$ is given by

$$
A_{n}(j \omega)=\sum_{i=1}^{R_{n}} h_{n}\left(k_{i}\right) Q_{n-k_{i}}(j \omega) e^{-j \omega k_{i}}
$$

where $Q_{n}(j \omega)$ represents the channel frequency response. In Paper F, we use a least-squares approach to determine the coefficients of each $h_{n}\left(k_{i}\right)$ through a matrix inversion of size $R_{n} \times R_{n}$. There, we assume that the TI-ADC suffers only from static time-skew errors which corresponds to $Q_{n}(j \omega)=$ $e^{j \omega \varepsilon_{n}}$. In Appendix B.1, using the least-squares approach, we derive a closedform expression for $h_{n}\left(k_{i}\right)$ that can be used to compensate general channel frequency response mismatches [20].

The constrained time-varying reconstructor requires $M M_{c}$ FIR filters ${ }^{6} h_{n}(k)$, $n \in\left[0,1, \ldots, M M_{c}-1\right]$, where the order of each filter, $N_{h_{n}}$, depends on how close the sample to be reconstructed is to the uniform-grid sample. Thus, the filter corresponding to the missing sample requires the highest order which can be seen from the design example in Paper F. For a given set of time-skew errors and $M_{c}$, the computational complexity of the reconstructor measured in terms of the number of multiplications per corrected output sample, is minimal for this reconstructor and is equal to $\sum_{n=0}^{M M_{c}-1} R_{n} / M M_{c}$. However, if the time-skew errors change, the coefficients of all the $M M_{c}$ filters need to be redetermined through $M M_{c}$ separate matrix inversion resulting in high online redesign complexity.

\footnotetext{
${ }^{6}$ In TI-ADC implementations, the mismatch parameters are estimated and reconstructed with respect to a reference channel. Thus, in a period of $M M_{c}$ samples, $M_{c}-1$ samples from the reference channel are available and require no correction. Hence, in practice, for the constrained time-varying reconstructor, we require only $M M_{c}-M_{c}+1$ separate FIR filters. However, in the design example in Section 4.2.4 as well as in Paper F of Part IV, we use all the $M M_{c}$ FIR filters to be able to compare with the corresponding example in [26].
} 


\subsubsection{Sub-Band Based Reconstructor}

The problem of reconstructing the uniform-grid samples from the output a TI-ADC with missing samples is similar to that of recovering the missing samples from a sub-Nyquist sampled sparse multi-band signal discussed in Chapter 3. Utilizing this observation, in Paper E, it is shown that the $M_{\mathrm{t}}$ periodically nonuniformly sampled signal at the output of an $M$-channel TIADC with missing samples can be performed using a set of $K$-channel analysis and synthesis FBs where $K=M_{\mathrm{t}}-M$. In the sub-band based reconstructor, the Nyquist band is divided into $M_{\mathrm{t}}$ sub-bands of equal length $\pi / M_{\mathrm{t}}$ and the first $K$ bands are assumed to be active. Due to the missing samples, only the inputs to the $K$ polyphase branches of the analysis filters $B_{k}\left(e^{j \omega}\right)$ are non-zero. Similar to the reconstructor in the sub-Nyquist CNUS case, here, the $K$ non-zero polyphase components $B_{k m_{\ell}}\left(e^{j \omega}\right), m_{\ell} \in\left[0,1, \ldots, M_{\mathrm{t}}-1\right]$, $\ell=1,2, \ldots, K$, approximate generalized fractional-delay filters so that

$$
B_{k m_{\ell}}\left(e^{j \omega}\right) \approx \beta_{k m_{\ell}} e^{j\left(\omega\left(m_{\ell}+\varepsilon_{\ell}\right) / M_{\mathrm{t}}+\alpha_{k m_{\ell}} \operatorname{sgn}(\omega)\right)}, \omega \in(-\pi, \pi)
$$

where $\beta_{k m_{\ell}}$ and $\alpha_{k m_{\ell}}$ are the modulus and angle, respectively, of a corresponding complex constant $c_{k m_{\ell}}$ which is determined using a matrix inversion, and $\varepsilon_{\ell}$ is the time-skew error corresponding to the sampling instant $m_{\ell}$.

The complexity of the online redesign block is reduced by expressing the polyphase components of all the analysis filters using a common set of $L+1$ fixed subfilters $F_{q}\left(e^{j \omega}\right)$ and $G_{q}\left(e^{j \omega}\right), q=0,1, \ldots, L$, such that [86]

$$
B_{k m_{\ell}}\left(e^{j \omega}\right)=\gamma_{k m_{\ell}} \sum_{q=0}^{L}\left(\frac{d_{\ell}}{M_{\mathrm{t}}}\right)^{q} F_{q}\left(e^{j \omega}\right)+\zeta_{k m_{\ell}} \sum_{q=0}^{L}\left(\frac{d_{\ell}}{M_{\mathrm{t}}}\right)^{q} G_{q}\left(e^{j \omega}\right)
$$

where $\gamma_{k m_{\ell}}=\beta_{k m_{\ell}} \cos \left(\theta_{k m_{\ell}}\right), \zeta_{k m_{\ell}}=\beta_{k m_{\ell}} \sin \left(\theta_{k m_{\ell}}\right), \theta_{k m_{\ell}}=\alpha_{k m_{\ell}}+\pi / 4$, $d_{\ell}=m_{\ell}+\varepsilon_{\ell}$, and $M_{\mathrm{t}}=M M_{c}$. Thus, the different polyphase branches can be obtained via different sets of values for $\gamma_{k m_{\ell}}, \zeta_{k m_{\ell}}$, and $d_{\ell}$. When the time-skew errors change, only the general multipliers corresponding to $\gamma_{k m_{\ell}}$, $\zeta_{k m_{\ell}}$, and $d_{\ell}$ need to be updated with the corresponding new values. Also, the new values of the constants $\gamma_{k m_{\ell}}$ and $\zeta_{k m_{\ell}}$ can be determined through a single $K \times K$ matrix inversion. The filters $F_{q}\left(e^{j \omega}\right)$ and $G_{q}\left(e^{j \omega}\right)$ are designed offline and are then fixed. In Paper $\mathrm{F}$, we propose a least-squares approach for determining the coefficients of these filters. ${ }^{7}$ Further, like in the subNyquist CNUS reconstructor, the bandpass filters in the synthesis FB are

\footnotetext{
${ }^{7}$ Appendix B.2 contains the detailed equations for the least-squares design of $F_{q}\left(e^{j \omega}\right)$ and $G_{q}\left(e^{j \omega}\right)$ filters.
} 
implemented using a cosine-modulated FB. The impulse response coefficients of the prototype filter for the cosine-modulated FB are determined offline and are then fixed.

The computational complexity of the sub-band based reconstructor can be evaluated as

$$
\mathcal{C}_{\mathrm{m}} \approx \frac{N_{P}}{M_{\mathrm{t}}}+\log _{2}\left(M_{\mathrm{t}}\right)+\frac{2(L+1) N_{F}}{M_{\mathrm{t}}}+\frac{2 L K}{M_{\mathrm{t}}}+\frac{2 K^{2}}{M_{\mathrm{t}}}
$$

where $N_{P}$ is the order of the prototype filter for the cosine-modulated FB and $N_{F}$ is the order of the fixed subfilters in the analysis FB. The first two terms in the expression for $\mathcal{C}_{\mathrm{m}}$ in (4.8), correspond to the computational complexity of the cosine-modulated synthesis FB assuming that the $2 M_{\mathrm{t}} \times M_{\mathrm{t}}$ transform block is implemented using a fast-transform algorithm [36]. The third, fourth, and fifth terms are the computational complexities of the $2(L+1)$ fixed subfilters $F_{q}\left(e^{j \omega}\right)$ and $G_{q}\left(e^{j \omega}\right)$, the $2 L$ multipliers $\left(d_{\ell} / M_{\mathrm{t}}\right)^{q}$, $q=1,2, \ldots, L$, and the $2 K^{2}$ multipliers corresponding to the scaled $\cos (\cdot)$ and $\sin (\cdot)$ terms in the analysis FB, respectively. As can be seen from the design example in Paper F, compared to the constrained time-varying reconstructor, the sub-band based reconstructor has higher computational complexity. However, in contrast to the time-varying reconstructor, the online redesign of the reconstructor requires only a single $K \times K$ matrix inversion.

\subsubsection{Pre-Filter Based Reconstructor}

As noted in Section 4.2.1, even though the constrained time-varying reconstructor has minimal computational complexity, the online redesign complexity is high due to the $M M_{c}$ matrix inversions that need to be performed online (see Footnote 6). Further, all the multipliers in the time-varying reconstructor should be implemented using expensive variable-coefficient multipliers as the coefficients of all the filters in the reconstructor can change during the online redesign.

In paper G of Part IV, we propose a pre-filter based reconstruction scheme shown here in Fig. 4.3. In this scheme, the overall implementation complexity is reduced by approximately recovering the missing samples using a pre-filter and then correcting the remaining mismatch errors using an iterative compensation structure. Since the pre-filter only approximately recovers the missing samples, its filter coefficients depend only on the location of the 


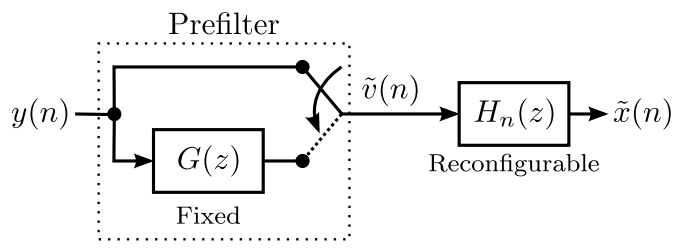

Figure 4.3: Prefilter-based reconstruction scheme.

Table 1: Complexity comparison for the different reconstruction schemes.

\begin{tabular}{|c|c|c|c|c|c|}
\hline Reconstructor & Order & \multicolumn{4}{|c|}{ Complexity $^{9}$} \\
& & $\mathcal{C}_{\mathrm{f}}$ & $\mathcal{C}_{\mathrm{v}}$ & $\mathcal{C}_{\mathrm{m}}$ & Redesign $^{10}$ \\
\hline \hline Recursive [26] & 396 & 543 & 11 & 554 & None \\
\hline Nonrecursive constrained & 68 & 0 & 31 & 31 & 28 \\
\hline Nonrecursive sub-band & 960 & 27 & 48 & 75 & 1 \\
\hline Nonrecursive pre-filter & 154 & 28 & 3 & 31 & None \\
\hline
\end{tabular}

missing samples and hence, can be determined offline and then fixed. The iterative structure consists of fixed subfilters and variable multipliers, like in [56]. Whenever the mismatch parameters change, the reconstructor can be reconfigured directly by updating the variable multipliers. As the coefficients of the pre-filter and subfilters in the compensation structure are determined offline and then fixed, they can be implemented using cheaper fixed-coefficient multipliers.

\subsubsection{Complexity Comparison}

Table 1 compares the complexities of the different reconstruction schemes when they are used to reconstruct the output of a four-channel TI-ADC $(M=4)$ in which every seventh sample is used to estimate the mismatch parameters $\left(M_{c}=7\right) .{ }^{8}$ It is assumed that the timing mismatches in the channel ADCs are $\varepsilon_{0,1,2,3}=[0.01,-0.05,0.04,-0.03]$ and the reconstructor bandwidth is $\omega_{\mathrm{c}}=0.8 \pi$. Further, it is required that the reconstructor should keep the aliasing terms below $-50 \mathrm{~dB}$. Here, to be able to compare with the

\footnotetext{
${ }^{8}$ Refer to Paper $\mathrm{G}$ for details on how the values in Table 1 were obtained.

${ }^{9} \mathcal{C}_{\mathrm{f}}$ and $\mathcal{C}_{\mathrm{v}}$ represent the number of fixed-coefficient and variable-coefficient multiplications per corrected output sample, respectively.

${ }^{10}$ Number of online matrix inversions.
} 
corresponding example in [26], the time-skew error of the reference channel, $\varepsilon_{0}$, is not assumed to be zero. It can be seen from Table 1 that the pre-filter based reconstructor requires the same number of multiplications per corrected output sample as the constrained time-varying reconstructor but with only fewer variable-coefficient multiplications per corrected output sample.

\subsection{Noise Gain}

All reconstruction schemes for TI-ADCs with missing samples amplify the noise at the input of the reconstructor. This noise gain is due to the presence of missing samples which restricts some of the impulse response coefficients of the reconstructor to be equal to zero. Consider, for example, the constrained time-varying FIR reconstructor used in the four-channel TI-ADC case in Section 4.2.4. However, here, for simplicity, we assume that the TI-ADC only contains missing samples and has no time-skew error. ${ }^{11}$ When there are no time-skew errors, the filters $H_{6}\left(e^{j \omega}\right), H_{13}\left(e^{j \omega}\right), H_{20}\left(e^{j \omega}\right)$, and $H_{27}\left(e^{j \omega}\right)$, which recover the missing samples can be linear-phase FIR filters. This also the case for the prefilter in the prefilter-based reconstructor where the noise gain is due to the linear-phase FIR filter $G(z)$ (see Fig. 4.3) which recovers the missing sample. Further, the reconstructor $H_{n}(z)$ in Fig. 4.3 only compensates for the mismatch errors and, hence, will not introduce any extra noise gain.

Assuming that $H_{6}\left(e^{j \omega}\right), H_{13}\left(e^{j \omega}\right), H_{20}\left(e^{j \omega}\right)$, and $H_{27}\left(e^{j \omega}\right)$ are noncausal filters, we have, $H_{n}\left(e^{j \omega}\right)=H_{n, \mathrm{R}}(\omega)$ where $H_{n, \mathrm{R}}(\omega), n \in[6,13,20,27]$, is the corresponding real-valued zero-phase frequency response (see Section 1.1.3). Also, the mid-tap impulse response coefficient $h_{n}(0), n \in[6,13,20,27]$ (and some other taps depending on $n$ and the order of $h_{n}(k)$ ), is equal to zero. The mid-tap value $h_{n}(0)$ can be represented in terms of its Fourier transform as

$$
h_{n}(0)=\frac{1}{2 \pi} \int_{-\pi}^{\pi} H_{n, \mathrm{R}}(\omega) \mathrm{d} \omega .
$$

Since $H_{n, \mathrm{R}}(\omega)=1$ for $\omega \in\left[-\omega_{\mathrm{c}}, \omega_{\mathrm{c}}\right]$, for the term on the right side of the equality in (4.9) to be equal to zero, $H_{n, \mathrm{R}}(\omega)$ must have negative values for

\footnotetext{
${ }^{11}$ The extra small time-skew errors will only have a very small effect on the noise gain analysis and conclusions.
} 
$|\omega| \in\left[\omega_{\mathrm{c}}, \pi\right]$. That is,

$$
\int_{\omega_{\mathrm{c}}}^{\pi} H_{n, \mathrm{R}}(\omega) \mathrm{d} \omega=A
$$

where $A=-\omega_{\mathrm{c}} / \pi$. Now, if $\sigma_{e}^{2}$ represents the noise power at the input of the filter $h_{n}(k)$, the noise power at the output of the filter is given by

$$
\sigma_{\tilde{x}_{e}}^{2}=\sigma_{e}^{2} \frac{1}{2 \pi} \int_{-\pi}^{\pi} H_{n, \mathrm{R}}^{2}(\omega) \mathrm{d} \omega
$$

Assuming ideal filters with $H_{n, \mathrm{R}}(\omega)=1$ in $|\omega| \in\left[0, \omega_{\mathrm{c}}\right]$, the minimum value of $H_{n, \mathrm{R}}(\omega)$ in $\omega \in\left(\omega_{\mathrm{c}}, \pi\right]$ can be determined by solving the problem:

$$
\operatorname{minimize} \int_{\omega_{\mathrm{c}}}^{\pi} H_{n, \mathrm{R}}^{2}(\omega) \mathrm{d} \omega \text { subject to } \int_{\omega_{\mathrm{c}}}^{\pi} H_{n, \mathrm{R}}(\omega) \mathrm{d} \omega=A .
$$

By dividing the frequency band $\omega \in\left(\omega_{\mathrm{c}}, \pi\right]$ into $W$ bands of width $\Delta \omega$, the integrals in (4.12) can be approximated using summations. Thus, (4.12) can be approximated as

$$
\operatorname{minimize} \Delta \omega \sum_{q=0}^{W-1}\left(H_{n, \mathrm{R}}^{(q)}(\omega)\right)^{2} \text { subject to } \Delta \omega \sum_{q=0}^{W-1} H_{n, \mathrm{R}}^{(q)}(\omega)=A
$$

where $H_{n, \mathrm{R}}^{(q)}(\omega)$ is the value of $H_{n, \mathrm{R}}(\omega)$ in the $q$ th band.

Now, since $\Delta \omega$ is a constant, $(4.13)$ can be restated as

$$
\operatorname{minimize} \sum_{q=0}^{W-1}\left(H_{n, \mathrm{R}}^{(q)}(\omega)\right)^{2} \text { subject to } \sum_{q=0}^{W-1} H_{n, \mathrm{R}}^{(q)}(\omega)=\frac{A}{\Delta \omega} .
$$

Since $H_{n, \mathrm{R}}(\omega)$ is real-valued, the function to be minimized can be rewritten as $[87]$

$$
\begin{aligned}
\sum_{q=0}^{W-1}\left(H_{n, \mathrm{R}}^{(q)}(\omega)\right)^{2}= & \frac{1}{W}\left(\sum_{q=0}^{W-1} H_{n, \mathrm{R}}^{(q)}(\omega)\right)^{2}+ \\
& \frac{1}{W} \sum_{q=0}^{W-2} \sum_{r=q+1}^{W-1}\left(H_{n, \mathrm{R}}^{(q)}(\omega)-H_{n, \mathrm{R}}^{(r)}(\omega)\right)^{2} \\
= & \frac{1}{W}\left(\frac{A}{\Delta \omega}\right)^{2}+\frac{1}{W} \sum_{q=0}^{W-2} \sum_{r=q+1}^{W-1}\left(H_{n, \mathrm{R}}^{(q)}(\omega)-H_{n, \mathrm{R}}^{(r)}(\omega)\right)^{2}
\end{aligned}
$$




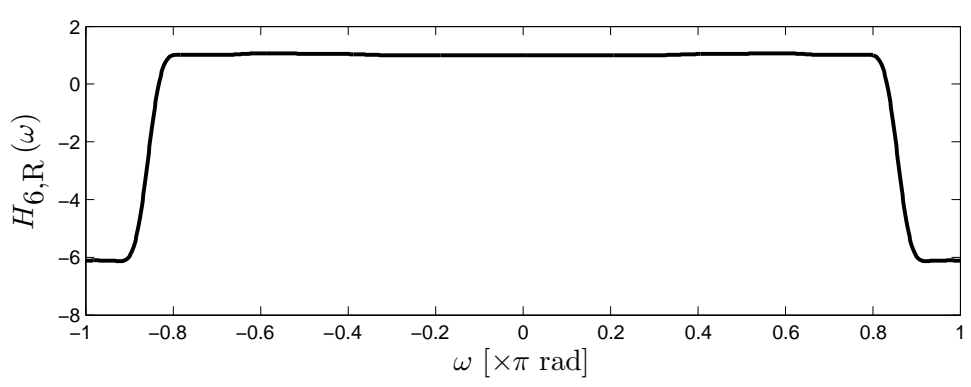

Figure 4.4: Frequency response of $H_{6, \mathrm{R}}(\omega)$ for the constrained time-varying reconstructor designed for the four-channel TI-ADC specification given in Section 4.2.4 where $\omega_{\mathrm{c}}=0.8 \pi$.

where the second equality in (4.15) is obtained by using the constraint in (4.14). It can be seen that the solution to (4.14) is obtained when the second term on the right side of the equality in (4.15) is equal to zero. For this, the value of $H_{n, \mathrm{R}}^{(q)}(\omega)$ in the $W$ frequency bands in $\omega \in\left(\omega_{\mathrm{c}}, \pi\right]$ should all be equal to a constant, say $A_{1}$. Thus,

$$
H_{n, \mathrm{R}}(\omega)= \begin{cases}1, & |\omega| \in\left[0, \omega_{\mathrm{c}}\right] \\ A_{1}, & |\omega| \in\left(\omega_{\mathrm{c}}, \pi\right] .\end{cases}
$$

Substituting (4.16) in (4.9) and solving for $h_{n}(0)=0$, we get

$$
A_{1}=-\frac{\omega_{\mathrm{c}}}{\pi-\omega_{\mathrm{c}}}
$$

This is also verified by Fig. 4.4 which plots the spectrum of $H_{6, \mathrm{R}}(\omega)$ for the constrained time-varying FIR reconstructor designed for the four-channel TI-ADC specification in Section 4.2.4 where $\omega_{\mathrm{c}}=0.8 \pi$. Using (4.16) in (4.11), we get the noise gain as

$$
\frac{\sigma_{\tilde{x}_{e}}^{2}}{\sigma_{e}^{2}}=\frac{\omega_{\mathrm{c}}}{\pi}+\frac{\omega_{c}^{2}}{\pi-\omega_{\mathrm{c}}} .
$$

Figure 4.5 illustrates how the noise gain at the output of the reconstruction filter $H_{n}\left(e^{j \omega}\right)$, used to recover the missing sample, increases with reconstructor bandwidth $\omega_{\mathrm{c}}$. It can be seen that the noise gain is not an issue in narrowband reconstructors but as $\omega_{\mathrm{c}}$ approaches $\pi$, the noise gain increases rapidly. For example, at $\omega_{\mathrm{c}}=0.8 \pi$, the noise gain at the output of the FIR 


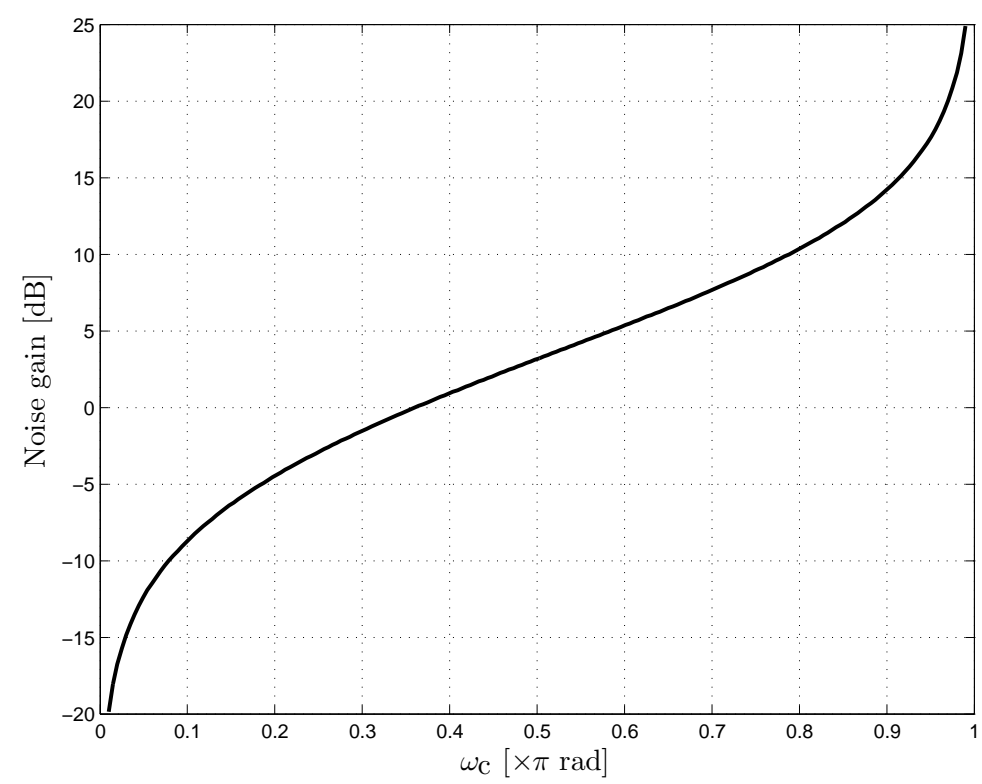

Figure 4.5: Noise gain at the output of the filter $H_{n}\left(e^{j \omega}\right)$ versus reconstructor bandwidth $\omega_{\mathrm{c}}$.

filter recovering the missing sample correspond to approximately 1.8 bits degradation in the resolution. However, the above noise gain affects only every $M_{c}$ th output sample and, hence, the average degradation at the output of the reconstructor will be less than 1.8 bits. Thus, if $M_{c}=7$, the noise gain will result in an average degradation of around 0.7 bits at the output of the reconstructor.

\subsection{Summary}

In this chapter, we introduced three reconstruction schemes that can be used to recover the uniform-grid samples from the nonuniformly sampled signal at the output of an unconventional TI-ADC. In the TI-ADC under consideration, some of the input sampling instants are reserved for estimating the channel mismatch parameters. Thus, in such TI-ADCs, nonuniform sampling is a result of the mismatch errors as well as the missing samples. Using a design example, the complexities of the three new schemes were compared 
with the existing iterative reconstruction scheme that uses recursive structures. It was seen that the proposed schemes provide around an order-ofmagnitude lower complexity compared to the existing iterative scheme. We also showed that, for reasonable bandwidths, the reconstruction schemes for TI-ADCs with missing samples have a noise gain greater than unity and that the magnitude of the noise gain is dependent on the bandwidth of the reconstructor. 


\section{Chapter 5}

\section{Summary of Specific Contributions of the Dissertation}

The contributions of this dissertation are divided into three parts: In Part II, we propose efficient signal reconstruction schemes for static time-skew error correction in TI-ADCs. The proposed schemes offer trade-offs between online redesign and reconstructor complexities. Part III of the dissertation deals with the reconstruction of sub-Nyquist sampled sparse multi-band signals which are obtained via CNUS. There, we propose an efficient reconfigurable reconstruction scheme that can potentially deliver an order-of-magnitude reduction in complexity compared to the existing scheme. In Part IV, we propose three reconstruction schemes for TI-ADCs in which some of the sampling instants are reserved for estimating the mismatch parameters. In addition to correcting the channel mismatch errors, the reconstructors used in such TI-ADCs should also recover the missing samples corresponding to the time instants reserved for estimation.

In the above parts, we provide the steps for designing the reconstructors. Design examples are used to compare the complexity of the proposed schemes with that of the existing schemes. 


\subsection{Included Papers}

Brief summaries of the papers included in this dissertation are as follows:

\section{Paper A: Two-Rate Based Low-Complexity Time-Varying Discrete-Time FIR Reconstructors for Two-Periodic Nonuni- formly Sampled Signals}

Authored by Anu Kalidas M. Pillai, and Håkan Johansson.

Published in Sampling Theory in Signal and Image Processing, 2013. Parts of this work have been presented at two conferences [88, 89].

This paper deals with time-varying finite-length impulse response (FIR) filters used for reconstruction of two-periodic nonuniformly sampled signals. The complexity of such reconstructors increases as their bandwidth approaches the whole Nyquist band. Reconstructor design that yields minimum reconstructor order requires expensive online redesign while those methods that simplify online redesign result in higher reconstructor complexity. This paper utilizes a two-rate approach to derive a single-rate structure where part of the complexity of the reconstructor is moved to a symmetric filter so as to reduce the number of multipliers. The symmetric filter is designed such that it can be used for all time-skew errors within a certain range, thereby reducing the number of coefficients that need online redesign. The basic two-rate based reconstructor is further extended to completely remove the need for online redesign at the cost of a slight increase in the total number of multipliers.

\section{Paper B: Efficient Signal Reconstruction Scheme for $M$-Channel Time-Interleaved ADCs}

Authored by Anu Kalidas M. Pillai, and Håkan Johansson.

Published in the Analog Integrated Circuits and Signal Processing, 2013.

In time-interleaved analog-to-digital converters (TI-ADCs), the timing mismatches between the channels result in a periodically nonuniformly sampled sequence at the output. Such nonuniformly sampled output limits the achievable resolution of the TI-ADC. In order to correct the errors due to timing mismatches, the output of the TI-ADC is passed through a digital time-varying finite-length impulse response (FIR) reconstructor. Such 
reconstructors convert the nonuniformly sampled output sequence to a uniformly spaced output. Since the reconstructor runs at the output rate of the TI-ADC, it is beneficial to reduce the number of coefficient multipliers in the reconstructor. Also, it is advantageous to have as few coefficient updates as possible when the timing errors change. Reconstructors that reduce the number of multipliers to be updated online do so at a cost of increased number of multiplications per corrected output sample. This paper proposes a technique which can be used to reduce the number of reconstructor coefficients that need to be updated online without increasing the number of multiplications per corrected output sample.

\section{Paper C: Low-Complexity Two-Rate Based Multivariate Impulse Response Reconstructor for Time-Skew Error Correction in $M$ - Channel Time-Interleaved ADCs}

Authored by Anu Kalidas M. Pillai, and Håkan Johansson.

Published in the proceedings of IEEE International Symposium on Circuits and Systems, 2013.

Nonuniform sampling occurs in time-interleaved analog-to-digital converters (TI-ADC) due to timing mismatches between the individual channel analog-to-digital converters (ADCs). Such nonuniformly sampled output will degrade the achievable resolution in a TI-ADC. To restore the degraded performance, digital time-varying reconstructors can be used at the output of the TI-ADC, which in principle, converts the nonuniformly sampled output sequence to a uniformly sampled output. As the bandwidth of these reconstructors increases, their complexity also increases rapidly. Also, since the timing errors change occasionally, it is important to have a reconstructor architecture that requires fewer coefficient updates when the value of the timing error changes. Multivariate polynomial impulse response reconstructor is an attractive option for an $M$-channel reconstructor. If the channel timing error varies within a certain limit, these reconstructors do not need any online redesign of their impulse response coefficients. This paper proposes a technique that can be applied to multivariate polynomial impulse response reconstructors in order to further reduce the number of fixed-coefficient multipliers, and thereby reduce the implementation complexity.

\section{Paper D: Efficient Recovery of Sub-Nyquist Sparse Multi-band Signals Using Reconfigurable Multi-Channel Analysis and Modu- lated Synthesis Filter Banks}


Authored by Anu Kalidas M. Pillai, and Håkan Johansson.

Submitted to the IEEE Transactions on Signal Processing, 2015. This is an extension of a conference publication [90].

Sub-Nyquist cyclic nonuniform sampling (CNUS) of a sparse multi-band signal generates a nonuniformly sampled signal. In order to recover the missing uniform-grid samples, the sequence obtained via CNUS is passed through a reconstructor. At present, these reconstructors have very high design and implementation complexity that offsets the gains obtained due to sub-Nyquist sampling. In this paper, we propose a scheme that reduces the design and implementation complexity of the reconstructor. In contrast to the existing reconstructors which use only synthesis filter bank (FB), the proposed reconstructor utilizes both analysis and synthesis FBs which makes it feasible to achieve an order-of-magnitude reduction of the complexity. The analysis filters are implemented using polyphase networks whose branches are allpass filters with distinct fractional delays and phase shifts. In order to reduce both the design and the implementation complexity of the synthesis FB, the synthesis filters are implemented using a cosine-modulated FB. In addition to the reduced complexity of the reconstructor, the proposed recovery scheme also supports online reconfigurability which is required in flexible (multi-mode) systems where the user subband locations vary with time.

\section{Paper E: A Sub-Band Based Reconstructor for $M$-Channel Time- Interleaved ADCs with Missing Samples}

Authored by Anu Kalidas M. Pillai, and Håkan Johansson.

Published in the proceedings of IEEE International Conference on Acoustics, Speech and Signal Processing, 2014.

This paper proposes a scheme for the recovery of a uniformly sampled sequence from the output of a time-interleaved analog-to-digital converter (TIADC) with static time-skew errors and missing samples. Nonuniform sampling occurs due to timing mismatches between the individual channel ADCs and also due to missing input samples as some of the sampling instants are reserved for estimating the mismatches in the TI-ADC. In addition to using a non-recursive structure, the proposed reconstruction scheme supports online reconfigurability and reduces the computational complexity of the reconstructor as compared to a previous solution. 
Paper F: Two Reconstructors for $M$-Channel Time Interleaved ADCs with Missing Samples

Authored by Anu Kalidas M. Pillai, and Håkan Johansson.

Published in the proceedings of IEEE International New Circuits and Systems Conference, 2014.

In this paper, we explore two nonrecursive reconstructors which recover the uniform-grid samples from the output of a time-interleaved analog-to-digital converter (TI-ADC) that uses some of the sampling instants for estimating the mismatches in the TI-ADC. Nonuniform sampling occurs due to timing mismatches between the individual channel ADCs and also due to missing input samples. Compared to a previous solution, the reconstructors presented here offer substantially lower computational complexity.

\section{Paper G: Prefilter-Based Reconfigurable Reconstructor for Time- Interleaved ADCs with Missing Samples}

Authored by Anu Kalidas M. Pillai, and Håkan Johansson.

Published in the IEEE Transactions on Circuits and Systems: II - Express Breifs, 2015.

This brief proposes a reconstruction scheme for the compensation of frequency-response mismatch errors at the output of a time-interleaved analog-to-digital converter (TI-ADC) with missing samples. The missing samples are due to sampling instants reserved for estimating the channel mismatch errors in the TI-ADC. Compared to previous solutions, the proposed scheme offers a substantially lower computational complexity.

\subsection{Not Included papers}

The following publications by the author are not included because they were earlier versions of the journal publications included in the dissertation.

[P1] A. K. M. Pillai and H. Johansson, "Efficient signal reconstruction scheme for time-interleaved ADCs," Proc. IEEE Int. New Circuits Syst. Conf., Montreal, Canada, Jun. 17-20, 2012, pp. 357-360. 
[P2] A. K. M. Pillai and H. Johansson, "Time-skew error correction in twochannel time-interleaved ADCs based on a two-rate approach and polynomial impulse responses," Proc. IEEE Int. Midwest Symp. Circuits Syst., Boise, ID, USA, Aug. 5-8, 2012, pp. 1136-1139.

[P3] A. K. M. Pillai and H. Johansson, "Efficient reconfigurable scheme for the recovery of sub-Nyquist sampled sparse multi-band signals," Proc. IEEE Global Conf. Signal Information Process., Austin, TX, USA, Dec. 3-5, 2013, pp. 1294-1297. 


\section{Appendix A}

\section{Alternative Derivation of the Reconstruction Scheme in Paper D}

Starting with the multi-level synthesis filters in [81], Paper D of Part III shows that the reconstruction can be described in terms of the analysis and synthesis filters. There, we also show that the non-zero polyphase components of the unconventional decimation filter $B_{k}(z)$ can be expressed as an allpass filter with a distinct fractional delay and a phase shift. In this appendix, however, we show this using a different approach which gives further insight and understanding of the efficient reconfigurable reconstruction scheme described in Paper D. For this purpose, we first consider conventional lowpass and bandpass filters used in resampling (interpolation and decimation). This is because the unconventional bandpass filters $B_{k}(z)$ can be viewed as extensions of the conventional bandpass filters whereas the polyphase components of the conventional bandpass filters in turn can be considered as a generalization of the polyphase components of lowpass filters. It is noted that lowpass filtering using polyphase networks was considered in [91], but here we use a different derivation which is suitable for the CNUS recovery problem. It will be shown that the conventional bandpass filter can be expressed in terms of a polyphase network whose branches are allpass filters with distinct fractional delays and phase shifts. Here, the phase shift values are directly given by one of the columns of a discrete Fourier transform (DFT) matrix. Extending the results derived for the conventional bandpass 
filter, we will then show that, for the unconventional bandpass filters $B_{k}(z)$, the gains of the allpass filters are generally different and the values of the phase shifts are not given by simple expressions but are obtained through a matrix inversion.

\section{A.1 Lowpass Filters}

Both interpolation and decimation require an (approximately) $M$ th-band filter with cutoff frequency at $\pi / M$. Recall that if $h(n)$ denotes the impulse response of the $M$ th-band lowpass filter, its transfer function, $H(z)$, is defined as

$$
H(z)=\sum_{n=-\infty}^{\infty} h(n) z^{-n} .
$$

The $M$-fold polyphase representation of $H(z)$ is given by [32]

$$
H(z)=\sum_{m=0}^{M-1} z^{-m} H_{m}\left(z^{M}\right)
$$

where

$$
H_{m}(z)=\sum_{n=-\infty}^{\infty} h(n M+m) z^{-n}
$$

denotes the $m$ th polyphase component of $H(z)$. In the lowpass decimation case, the (noncausal) filter's frequency response $H\left(e^{j \omega}\right)$ is ideally unity in the passband $|\omega| \in[0, \pi / M]$ and zero in the stopband $|\omega| \in(\pi / M, \pi]$.

Next, we will show that the $M$ th-band lowpass filter $H\left(e^{j \omega}\right)$ is obtained if its polyphase components are fractional-delay (FD) filters with distinct FD values [91]. It is noted that, here we show only the "if" part. The "only if" part can be shown as in [92]. Here, we divide the frequency range $[-\pi / M, 2 \pi-\pi / M]$ into $M$ adjacent regions of equal width $2 \pi / M$ as shown in Fig. A.1(a). Thus, region $p, p \in[0,1, \ldots, M-1]$, covers the frequencies in $[-\pi / M+2 \pi p / M,-\pi / M+2 \pi(p+1) / M]$. Next, it is assumed that $H_{m}\left(e^{j \omega}\right)$ are FD filters according to

$$
H_{m}\left(e^{j \omega}\right)=\frac{1}{M} e^{j \omega m / M}, \omega \in[-\pi, \pi]
$$

and $H_{m}\left(e^{j \omega}\right)$ are $2 \pi$-periodic with respect to $\omega$. The latter feature implies that the frequency response of the polyphase component for any $\omega \in[-\pi+$ 


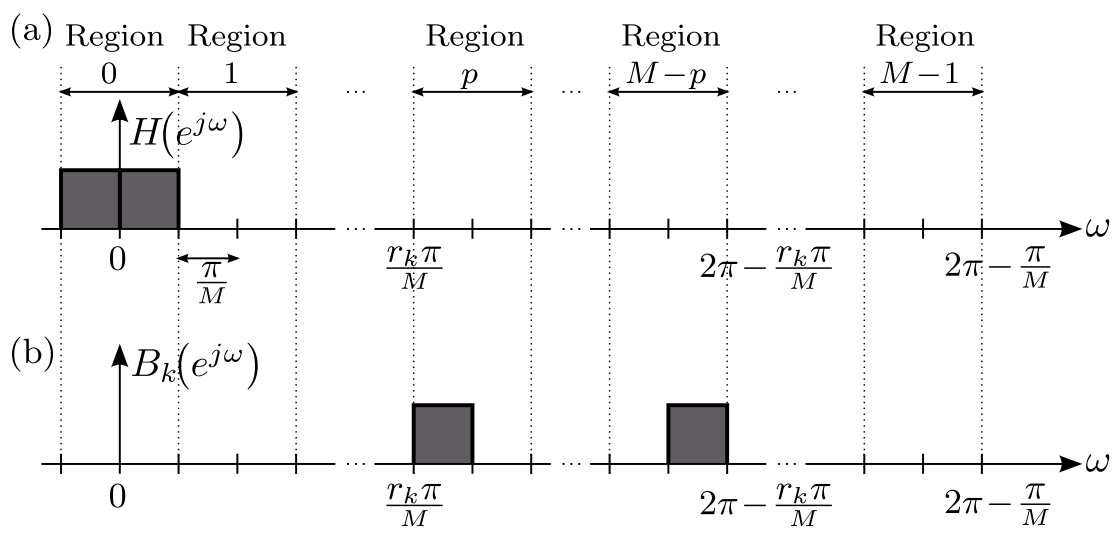

Figure A.1: (a) Spectrum of a lowpass filter with cutoff frequency at $\pi / M$. The frequency range $[-\pi / M, 2 \pi-\pi / M]$ is divided into $M$ adjacent regions of equal width $2 \pi / M$. (b) Spectrum of a bandpass filter with passband in the frequency range $\left[r_{k} \pi / M,\left(r_{k}+1\right) \pi / M\right]$.

$2 \pi p,-\pi+2 \pi(p+1)]$ and all integers $p$ should be equal to $H_{m}\left(e^{j \omega}\right)$ in $\omega \in$ $[-\pi, \pi]$. Hence, $H_{m}\left(e^{j \omega}\right)=e^{j(\omega-2 \pi p) m / M} / M$ for $\omega \in[-\pi+2 \pi p,-\pi+2 \pi(p+$ 1)] and all integers $p$. It is further noted that $H_{m}\left(e^{j \omega M}\right)$ are compressed (by $M$ ) versions of the corresponding frequency responses $H_{m}\left(e^{j \omega}\right)$. This means that $H_{m}\left(e^{j \omega M}\right)$ for $\omega \in[-\pi / M+2 \pi p / M,-\pi / M+2 \pi(p+1) / M]$ equals $H_{m}\left(e^{j \omega}\right)$ for $\omega \in[-\pi+2 \pi p,-\pi+2 \pi(p+1)]$. Thus, $H_{m}\left(e^{j \omega M}\right)=$ $e^{j(\omega M-2 \pi p) m / M} / M$ for $\omega \in[-\pi / M+2 \pi p / M,-\pi / M+2 \pi(p+1) / M]$ and all integers $p$.

For a real lowpass filter with a cutoff frequency at $\pi / M$ and a passband gain of unity, we require that $H\left(e^{j \omega}\right)=1$ for $p=0$ and $H\left(e^{j \omega}\right)=0$ for $p=1,2, \ldots, M-1$. Since $H\left(e^{j \omega}\right)$ is $2 \pi$-periodic, it suffices to consider $p=0,1, \ldots, M-1$ as this corresponds to a frequency range of $2 \pi$. Using $H_{m}\left(e^{j \omega M}\right)=e^{j(\omega m-2 \pi p m / M)} / M$ in (A.2), we obtain

$$
\begin{aligned}
H\left(e^{j \omega}\right) & =\frac{1}{M} \sum_{m=0}^{M-1} e^{-j \omega m} e^{j(\omega m-2 \pi p m / M)} \\
& = \begin{cases}1, & p=0 \\
0, & p=1,2, \ldots, M-1 .\end{cases}
\end{aligned}
$$

Thus, the FD polyphase components in (A.4) results in an overall lowpass filter with cutoff frequency at $\pi / M$. 


\section{A.2 Conventional Bandpass Filters}

Next, we will focus on the bandpass filter case where the passband of the desired real bandpass filter $B_{k}\left(e^{j \omega}\right)$ is assumed to cover the band $\omega \in\left[r_{k} \pi / M,\left(r_{k}+1\right) \pi / M\right], r_{k} \in[0,1, \ldots, M-1]$, and thus also $\omega \in$ $\left[2 \pi-\left(r_{k}+1\right) \pi / M, 2 \pi-r_{k} \pi / M\right]$ as shown in Fig. A.1(b). Regarding the transfer function, the point of departure is again the polyphase representation in (A.2). However, to obtain a bandpass filter, we cannot use the FD polyphase component in (A.4) since this choice automatically results in a lowpass filter as shown in Appendix A.1. As will be shown below, a bandpass filter is obtained by adding phase offsets to the FD filters in (A.4). In other words, we replace (A.4) with

$$
B_{k m}\left(e^{j \omega}\right)=\frac{1}{M} e^{j\left(\omega m / M+\alpha_{k m} \operatorname{sgn}(\omega)\right)}, \omega \in[-\pi, \pi]
$$

where $\operatorname{sgn}(\omega)$ is the sign of $\omega$. Again, we assume that the frequency range $[-\pi / M, 2 \pi-\pi / M]$ is divided into $M$ adjacent regions of equal width $2 \pi / M$ as shown in Fig. A.1(b). Thus, if $B_{k}\left(e^{j \omega}\right)$ occupies the left (right) half of the region $p, p \in[0,1, \ldots, M-1]$, it will also occupy the right (left) half of the $M-p$ th region as shown in Fig. A.1(b). Comparing Figs. A.1(a) and A.1(b), it can be seen that, unlike in the lowpass filter case where $H\left(e^{j \omega}\right)$ equals unity in the whole region 0 , here, $B_{k}\left(e^{j \omega}\right)$ should not be equal to unity in the whole region $p$. Instead, as the passband of a real bandpass decimation filter cover only half of such a region, we get two requirements per region $p$. As will be shown below, if we satisfy the two requirements for region $p$, we will also automatically satisfy the two requirements for region $M-p$. Hence, in total, we will end up with a system of $M$ equations with $M$ unknowns $\alpha_{k m}$. Also, it will be shown that the values of $\alpha_{k m}$ are obtained as one of the columns in a scaled DFT matrix.

The difference from the lowpass case is that, due to the $\operatorname{sgn}(\omega)$ in (A.6), $B_{k m}\left(e^{j \omega M}\right)=e^{j\left(\omega m-2 \pi p m / M-\alpha_{k m}\right)} / M$ in the left part of region $p$ whereas $B_{k m}\left(e^{j \omega M}\right)=e^{j\left(\omega m-2 \pi p m / M+\alpha_{k m}\right)} / M$ in the right part of the same region. Using these expressions in (A.2), we get

$$
B_{k}\left(e^{-j \omega}\right)=\frac{1}{M} \sum_{m=0}^{M-1} e^{j 2 \pi p m / M} e^{j \alpha_{k m}}
$$

for $\omega \in[-\pi / M+2 \pi p / M, 2 \pi p / M]$ (left part of region $p$ ) and

$$
B_{k}\left(e^{j \omega}\right)=\frac{1}{M} \sum_{m=0}^{M-1} e^{-j 2 \pi p m / M} e^{j \alpha_{k m}}
$$


for $\omega \in[2 \pi p / M,-\pi / M+2 \pi(p+1) / M]$ (right part of region $p$ ). Above, we used $B_{k}\left(e^{-j \omega}\right)$ in (A.7) since real filters are assumed. For real filters, $B_{k}\left(e^{j \omega}\right)=1\left(B_{k}\left(e^{j \omega}\right)=0\right)$ in the passband (stopband) region implies $B_{k}\left(e^{-j \omega}\right)=1\left(B_{k}\left(e^{-j \omega}\right)=0\right)$ as well.

It can be seen that (A.7) and (A.8) also correspond to the right and the left half, respectively, of region $M-p$. Hence, the two requirements on $B_{k}\left(e^{j \omega}\right)$ in region $p$ equals the two requirements in region $M-p$. Consequently, it suffices to solve a system of $M$ equations for $M$ values of $q= \pm p$ that correspond to the frequency band $\omega \in[0, \pi]$. If $M$ is even, these values are $q=0, \pm 1, \pm 2, \ldots, M / 2$ and if $M$ is odd, we have $q=0, \pm 1, \pm 2, \ldots, \pm(M-$ $1) / 2$. For convenience, we will however restate the requirements in terms of positive values of $q$ which is possible because $e^{-j 2 \pi p m / M}=e^{j 2 \pi(M-p) m / M}$. Then, $q=0,1,2, \ldots, M-1$, and they correspond to the left half of the $M$ regions in Fig. A.1(b). Thus, we obtain the system of equations

$$
\mathbf{D}_{k}=\mathbf{b}_{k}
$$

where $\mathbf{D}$ is an $M \times M$ matrix with the elements $d_{q m}=\frac{1}{M} e^{j 2 \pi q m / M}, q, m=$ $0,1, \ldots, M-1$, and $\mathbf{v}_{k}$ is a vector $\left(M \times 1\right.$ matrix) with $M$ unknowns $v_{k, m}=$ $e^{j \alpha_{k m}}, m=0,1, \ldots, M-1$. Further, $\mathbf{b}_{k}$ is a vector $(M \times 1$ matrix $)$ with elements $b_{k, m}, m=0,1, \ldots, M-1$, that correspond to the required frequency response in the left half of the $M$ subbands which will result in an overall $B_{k}\left(e^{j \omega}\right)$ shown in Fig. A.1(b). That is, $\mathbf{b}_{k}$ contains $M-1$ zeros and unity for the position corresponding to the passband $\omega \in\left[r_{k} \pi / M,\left(r_{k}+1\right) \pi / M\right]$, $r_{k} \in[0,1, \ldots, M-1]$. Assuming that $b_{k, w\left(r_{k}\right)}=1$, the value of $w\left(r_{k}\right)$ is given by

$$
w\left(r_{k}\right)= \begin{cases}\frac{r_{k}+1}{2}, & \text { odd } r_{k} \\ M-\frac{r_{k}}{2}, & \text { even } r_{k} \neq 0 . \\ 0, & r_{k}=0\end{cases}
$$

It is noted that $\mathbf{D}$ is the inverse discrete Fourier transform (IDFT) matrix. Therefore, the inverse of $\mathbf{D}$ always exists and equals the DFT matrix. Since $b_{k, w\left(r_{k}\right)}=1$ and $b_{k, m}=0$ for $m \neq w\left(r_{k}\right), v_{k, m}$ are obtained as

$$
v_{k, m}=e^{-j 2 \pi w\left(r_{k}\right) m / M}, m=0,1, \ldots, M-1 .
$$

Consequently, the phase offsets $\alpha_{k m}$ in (A.6) become

$$
\alpha_{k m}=\frac{-2 \pi w\left(r_{k}\right) m}{M}, m=0,1, \ldots, M-1 .
$$

Thus, with $\alpha_{k m}$ as in (A.12), the polyphase components in (A.6) result in an overall bandpass filter with passband in the region $\omega \in\left[r_{k} \pi / M, \quad\left(r_{k}+\right.\right.$ 1) $\pi / M]$. 

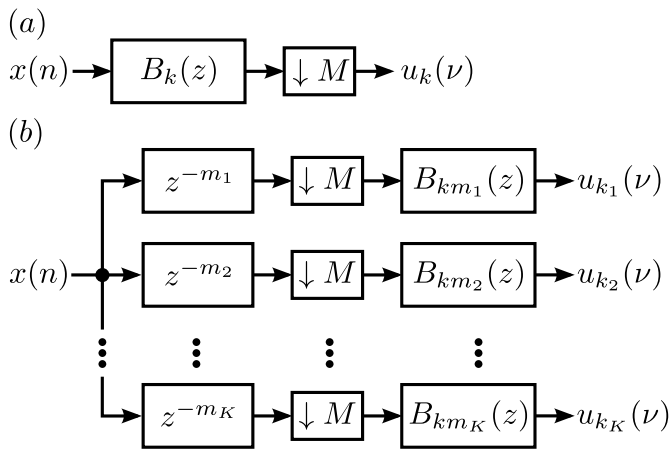

Figure A.2: (a) Bandpass decimator in the $k$ th branch of the analysis FB in the proposed reconstructor. (b) Polyphase representation of the bandpass decimator in (a) when $x(n)$ is obtained via sub-Nyquist CNUS.

\section{A.3 Unconventional Bandpass Filters}

We now extend the above results to bandpass filters used in the analysis FB of the proposed reconstructor (Fig. 3.5). Figure A.2(a) shows the $k$ th branch of the analysis FB in the proposed reconstructor. Recall that since the input signal is sub-Nyquist sampled as explained in Section 3.1, it can be considered that the available input samples $u_{k}(\nu)=x\left(M \nu-m_{\ell}\right), \ell=$ $1,2, \ldots, K$, are obtained from the uniform-grid samples $x(n)$ as shown in Fig. 3.2(a). Thus, due to missing samples, the inputs to $M-K$ polyphase branches of the bandpass filter $B_{k}(z)$ in Fig. A.2(a) will be equal to zero. This implies that

$$
B_{k}(z)=\sum_{\ell=1}^{K} z^{-m_{\ell}} B_{k m_{\ell}}\left(z^{M}\right)
$$

where $m_{\ell} \in[0,1, \ldots, M-1], \ell=1,2, \ldots, K$, are the $K$ sampling instants and $B_{k m_{\ell}}\left(e^{j \omega}\right)$ are the $K$ non-zero polyphase components of $B_{k}(z)$. Hence, the bandpass decimator in Fig. A.2(a) can be redrawn as shown in Fig. A.2(b). Similar to the bandpass filter in Appendix A.2, the polyphase components of the bandpass filter $B_{k}(z)$ are generalized FD filters but, here, the non-zero polyphase components $B_{k m_{\ell}}\left(e^{j \omega}\right)$ can be written as

$$
B_{k m_{\ell}}\left(e^{j \omega}\right) \approx \frac{\beta_{k m_{\ell}}}{M} e^{j\left(\omega m_{\ell} / M+\alpha_{k m_{\ell}} \operatorname{sgn}(\omega)\right)}, \omega \in(-\pi, \pi) .
$$

In (A.14), $\beta_{k m_{\ell}}$ and $\alpha_{k m_{\ell}}$ are the modulus and angle, respectively, of a corresponding complex constant $v_{k m_{\ell}}$. Moreover, proceeding in the same way 
as in Appendix A.2, the vector $\mathbf{v}_{k}$, containing all the $K$ complex constants $v_{k m_{\ell}}, m_{\ell} \in[0,1, \ldots, M-1], \ell=1,2, \ldots, K$, can be determined using matrix inversion as

$$
\mathbf{v}_{k}=\mathbf{D}^{-1} \mathbf{b}_{k}
$$

Here, $\mathbf{D}$ is a $K \times K$ generalized Vandermonde matrix whose elements are determined by the $K$ sampling points $m_{\ell}$ and the $K$ regions $q_{i}$, whose left halves are occupied by the active subbands $r_{i}$, such that

$$
\mathbf{D}=\frac{1}{M}\left[\begin{array}{cccc}
e^{j 2 \pi q_{1} m_{1} / M} & e^{j 2 \pi q_{1} m_{2} / M} & \cdots & e^{j 2 \pi q_{1} m_{K} / M} \\
e^{j 2 \pi q_{2} m_{1} / M} & e^{j 2 \pi q_{2} m_{2} / M} & \cdots & e^{j 2 \pi q_{2} m_{K} / M} \\
\vdots & \vdots & \ddots & \vdots \\
e^{j 2 \pi q_{K} m_{1} / M} & e^{j 2 \pi q_{K} m_{2} / M} & \cdots & e^{j 2 \pi q_{K} m_{K} / M}
\end{array}\right]
$$

where $q_{i}, m_{\ell} \in[0,1, \ldots, M-1], i, \ell=1,2, \ldots, K$. It can be seen from (A.16) that there is always at least one set of sampling instants that corresponds to an invertible matrix, namely $m_{\ell}=0,1, \ldots, K$, since for these sampling points the generalized Vandermonde matrix $\mathbf{D}$ reduces to a Vandermonde matrix. However, these sampling instants may not guarantee that the matrix $\mathbf{D}$ is well conditioned. In order to ensure that $\mathbf{D}$ is well conditioned, optimal sampling instants can be selected depending on the active subband locations as outlined in $[82,84]$. Further, $\mathbf{b}_{k}$ is a vector $(K \times 1$ matrix) containing $K-1$ zeros and unity for the position $k$. 


\section{Appendix B}

\section{Derivation of the Least-Squares Design in Paper F}

In Paper F of Part IV, we propose least-squares design for the two reconstructors used in TI-ADCs with missing samples. In this appendix, we provide the derivation of these least-squares designs since the derivations were not included in Paper F due to space limitations.

\section{B.1 Constrained Time-Varying FIR Reconstructor}

In Paper F, the least-squares design proposed for the constrained timevarying reconstructor assumes that the channel ADCs have only static timeskew errors. However, here, we consider the more general case where each channel ADC has a frequency response $Q_{n}(j \omega)$. The expression for the static time-skew error case considered in Paper F can be obtained by substituting $Q_{n}(j \omega)=e^{j \omega \varepsilon_{n}}$.

Let $\mathbf{h}_{n}$ represent the vector $\left(R_{n} \times 1\right.$ matrix) containing the $R_{n}$ non-zero impulse response coefficients in $h_{n}(k)$ such that

$$
\mathbf{h}_{n}=\left[h_{n}\left(k_{1}\right) h_{n}\left(k_{2}\right) \ldots h_{n}\left(k_{R_{n}}\right)\right]^{T} .
$$


Now, $\tilde{\mathbf{h}}_{n}$, the vector $\left(N_{h_{n}}+1 \times 1\right.$ matrix $)$ containing all the impulse response coefficients of $h_{n}(k)$ can be written as

$$
\tilde{\mathbf{h}}_{n}=\mathbf{D}_{n} \mathbf{h}_{n}
$$

where $\mathbf{D}_{n}$ is a $N_{h_{n}}+1 \times R_{n}$ matrix given by

$$
\mathbf{D}_{n}=\left[\begin{array}{llll}
\mathbf{d}_{n, 1} & \mathbf{d}_{n, 2} & \ldots & \mathbf{d}_{n, R_{n}}
\end{array}\right],
$$

with

$$
\mathbf{d}_{n, i}=\left[\begin{array}{llll}
d_{n,-N_{h_{n}} / 2}^{(i)} & d_{n,-N_{h_{n}} / 2+1}^{(i)} \ldots & d_{n, N_{h_{n}} / 2}^{(i)}
\end{array}\right]^{T}
$$

and

$$
d_{n, k}^{(i)}= \begin{cases}1, & k=k_{i} \\ 0, & \text { otherwise }\end{cases}
$$

for $i=1,2, \ldots, R_{n}, k=-N_{h_{n}} / 2,-N_{h_{n}} / 2+1, \ldots, N_{h_{n}} / 2$. Then, assuming that $x_{a}(t)$ is bandlimited to $|\omega| \leq \omega_{0}<\pi$, the error power function

$$
\mathrm{P}_{n}=\frac{1}{2 \pi} \int_{-\omega_{0}}^{\omega_{0}}\left|A_{n}(j \omega)-1\right|^{2} \mathrm{~d} \omega
$$

can be expressed in terms of the non-zero impulse response coefficients $\mathbf{h}_{n}$ as

$$
\begin{aligned}
\mathrm{P}_{n} & =\tilde{\mathbf{h}}_{n}^{T} \mathbf{S}_{n} \tilde{\mathbf{h}}_{n}-2 \mathbf{b}_{n}^{T} \tilde{\mathbf{h}}_{n}+\frac{\omega_{0}}{\pi} \\
& =\mathbf{h}_{n}^{T} \mathbf{D}_{n}^{T} \mathbf{S}_{n} \mathbf{D}_{n} \mathbf{h}_{n}-2 \mathbf{b}_{n}^{T} \mathbf{D}_{n} \mathbf{h}_{n}+\frac{\omega_{0}}{\pi}
\end{aligned}
$$

where $\mathbf{S}_{n}$ is an $N_{h_{n}}+1 \times N_{h_{n}}+1$ matrix with elements $S_{n, k p}, k, p=$ $-N_{h_{n}} / 2,-N_{h_{n}} / 2+1, \ldots, N_{h_{n}} / 2$, given by

$$
\begin{aligned}
S_{n, k p}= & \frac{1}{2 \pi} \int_{-\omega_{0}}^{\omega_{0}}\left|Q_{n-k}(j \omega)\right|\left|Q_{n-p}(j \omega)\right| \\
& \times \cos \left(\omega(p-k)+\arg \left\{Q_{n-k}(j \omega)\right\}-\arg \left\{Q_{n-k}(j \omega)\right\}\right) \mathrm{d} \omega
\end{aligned}
$$

and

$$
\mathbf{b}_{n}=\left[\begin{array}{llll}
b_{n,-N_{h_{n}} / 2} & b_{n,-N_{h_{n}} / 2+1} & \ldots & b_{n, N_{h_{n}} / 2}
\end{array}\right]^{T}
$$

with

$$
b_{n, k}=\frac{1}{2 \pi} \int_{-\omega_{0}}^{\omega_{0}}\left|Q_{n-k}(j \omega)\right| \cos \left(\omega-\arg \left\{Q_{n-k}(j \omega)\right\}\right) \mathrm{d} \omega
$$


for $k=-N_{h_{n}} / 2,-N_{h_{n}} / 2+1, \ldots, N_{h_{n}} / 2$. The value of $\mathbf{h}_{n}$ that minimizes $\mathrm{P}_{n}$ in (B.7) is obtained by solving

$$
\frac{\partial \mathrm{P}_{n}}{\partial \mathbf{h}_{n}}=0
$$

which gives

$$
\mathbf{h}_{n}=\left(\mathbf{D}_{n} \mathbf{S}_{n}\right)^{-1} \mathbf{b}_{n}^{T}
$$

\section{B.2 Least-Squares Design of $F_{q}\left(e^{j \omega}\right)$ and $G_{q}\left(e^{j \omega}\right)$ in the Sub-band Based Reconstructor}

Here, we derive the closed-form expression for determining the impulse response coefficients of the subfilters $F_{q}\left(e^{j \omega}\right)$ and $G_{q}\left(e^{j \omega}\right)$ in the analysis FB of the sub-band based reconstructor in Paper F. The closed-form expression is derived using the least-squares design outlined in Section 4.1 of Paper F.

Let $N_{F}$ denote the order of the $2(L+1)$ subfilters $F_{q}\left(e^{j \omega}\right)$ and $G_{q}\left(e^{j \omega}\right)$, $q=0,1, \ldots, L$, in the analysis $\mathrm{FB}$ and $N_{C}$ the order of the synthesis filters $C_{k}\left(e^{j \omega}\right)$. In the following equations, for simplicity, we assume that the filters are noncausal with even order. Then, the vectors $\left(N_{F}+1 \times 1\right.$ matrices $) \mathbf{f}_{q}$ and $\mathbf{f}_{q}$ containing the impulse response coefficients of $F_{q}\left(e^{j \omega}\right)$ and $G_{q}\left(e^{j \omega}\right)$, respectively, are given by

$$
\mathbf{f}_{q}=\left[\begin{array}{llll}
f_{q}\left(-N_{F} / 2\right) & f_{q}\left(-N_{F} / 2+1\right) & \ldots & f_{q}\left(N_{F} / 2\right)
\end{array}\right]^{T}
$$

and

$$
\mathbf{g}_{q}=\left[\begin{array}{llll}
g_{q}\left(-N_{F} / 2\right) & g_{q}\left(-N_{F} / 2+1\right) & \ldots & g_{q}\left(N_{F} / 2\right)
\end{array}\right]^{T}
$$

where $f_{q}(n)$ and $g_{q}(n), n=-N_{F} / 2,-N_{F} / 2+1, \ldots, N_{F} / 2$, are the impulse response coefficients of $F_{q}\left(e^{j \omega}\right)$ and $G_{q}\left(e^{j \omega}\right)$, respectively. Similarly, the vector $\left(N_{C}+1 \times 1\right.$ matrix) $\mathbf{c}_{k}$ containing the impulse response coefficients of $C_{k}\left(e^{j \omega}\right)$ is given by

$$
\mathbf{c}_{k}=\left[\begin{array}{llll}
c_{k}\left(-N_{C} / 2\right) & c_{k}\left(-N_{C} / 2+1\right) & \ldots & c_{k}\left(N_{C} / 2\right)
\end{array}\right]^{T}
$$

where $c_{k}(n), n=-N_{C} / 2,-N_{C} / 2+1, \ldots, N_{C} / 2$, are the impulse response coefficients of $C_{k}\left(e^{j \omega}\right)$. Then, the distortion function $(p=0)$ and aliasing 
functions $(p=1,2, \ldots, N-1), V_{p}\left(e^{j \omega}\right)$ in (10) of paper $\mathrm{F}$ can be expressed in matrix form as ${ }^{12}$

$$
V_{p}\left(e^{j \omega}\right)=\frac{1}{N} \mathbf{e}\left(\omega, N_{C}\right) \mathbf{C U}(\omega, p) \mathbf{h}
$$

where

$$
\begin{aligned}
& \mathbf{e}\left(\omega, N_{C}\right)=\left[\begin{array}{llll}
e^{-j \omega\left(-N_{C} / 2\right)} & e^{-j \omega\left(-N_{C} / 2+1\right)} & \ldots & e^{-j \omega N_{C} / 2}
\end{array}\right], \\
& \mathbf{C}=\left[\begin{array}{llll}
\mathbf{c}_{1} & \mathbf{c}_{2} & \ldots & \mathbf{c}_{K}
\end{array}\right], \\
& \mathbf{U}(\omega, p)=\left[\begin{array}{llll}
\mathbf{u}_{1}^{T} & \mathbf{u}_{2}^{T} & \ldots & \mathbf{u}_{K}^{T}
\end{array}\right]^{T},
\end{aligned}
$$

and

$$
\mathbf{h}=\left[\begin{array}{lllllll}
\mathbf{f}_{0}^{T} & \mathbf{g}_{0}^{T} & \mathbf{f}_{1}^{T} & \mathbf{g}_{1}^{T} & \ldots & \mathbf{f}_{L}^{T} & \mathbf{g}_{L}^{T}
\end{array}\right]^{T}
$$

In (B.19)

$$
\mathbf{u}_{k}(\omega, p)=\sum_{\ell=1}^{K} \mathbf{d}_{k \ell}(\omega, p)
$$

for $k=1,2, \ldots, K$, with

$$
\begin{aligned}
& \mathbf{d}_{k \ell}(\omega, p)=\left[\begin{array}{lllll}
\mathbf{a}_{k \ell}^{(0)}(\omega, p) & \mathbf{b}_{k \ell}^{(0)}(\omega, p) & \ldots & \mathbf{a}_{k \ell}^{(K)}(\omega, p) & \mathbf{b}_{k \ell}^{(K)}(\omega, p)
\end{array}\right], \\
& \mathbf{a}_{k \ell}^{(q)}(\omega, p)=a_{k \ell}(\omega, p) \mathbf{e}\left(\omega N, N_{F}\right)\left(\frac{d_{\ell}}{N}\right)^{q}, \\
& \mathbf{b}_{k \ell}^{(q)}(\omega, p)=b_{k \ell}(\omega, p) \mathbf{e}\left(\omega N, N_{F}\right)\left(\frac{d_{\ell}}{N}\right)^{q}
\end{aligned}
$$

for $q=0,1, \ldots, L$,

$$
a_{k \ell}(\omega, p)=\gamma_{k m_{\ell}} e^{-j(\omega-2 \pi p / N) d_{\ell}},
$$

and

$$
b_{k \ell}(\omega, p)=\zeta_{k m_{\ell}} e^{-j(\omega-2 \pi p / N) d_{\ell}},
$$

where $\gamma_{k m_{\ell}}$ and $\zeta_{k m_{\ell}}$ are as in Section 4 of Paper F. The vector $\mathbf{e}\left(\omega N, N_{F}\right)$ in (B.23) and (B.24) is obtained by replacing $\omega$ and $N_{C}$ in (B.17) with $\omega N$ and $N_{F}$, respectively. With $V_{p}\left(e^{j \omega}\right)$ as in (B.16), from (12) in Paper F, the error power function $\mathrm{P}_{0}$ can be written as

$$
\mathrm{P}_{0}=\frac{1}{N^{2}} \mathbf{h}^{T} \mathbf{S}_{0} \mathbf{h}-\frac{2}{N^{2}} \mathbf{r}_{0} \mathbf{h}+\frac{1}{N^{2}} \frac{\omega_{0}}{\pi}
$$

\footnotetext{
${ }^{12}$ Here, $N$ corresponds to $M_{\mathrm{t}}$ in Section 4.2 .
} 
and $\mathrm{P}_{p}, p=1,2, \ldots, N-1$, become

$$
\mathrm{P}_{p}=\frac{1}{N^{2}} \mathbf{h}^{T} \mathbf{S}_{p} \mathbf{h}
$$

where

$$
\mathbf{S}_{p}=\frac{1}{2 \pi} \int_{\omega_{0}}^{\omega_{0}} \mathbf{U}^{\dagger}(\omega, p) \mathbf{C}^{T} \mathbf{e}^{\dagger}\left(\omega, N_{C}\right) \mathbf{e}\left(\omega, N_{C}\right) \mathbf{C U}(\omega, p) \mathrm{d} \omega
$$

for $p=0,1, \ldots, N-1$, and

$$
\mathbf{r}_{0}=\frac{1}{2 \pi} \int_{\omega_{0}}^{\omega_{0}} \operatorname{Re}\left\{\mathbf{e}\left(\omega, N_{C}\right) \mathbf{C U}(\omega, 0)\right\} \mathrm{d} \omega .
$$

Then, the $\mathbf{h}$ that minimizes $\mathrm{P}$ in (11) of Paper $\mathrm{F}$ can be found by solving $\partial \mathrm{P} / \partial \mathbf{h}$ and is given by

$$
\mathbf{h}=\left[\sum_{p=0}^{N-1} \mathbf{S}_{p}\right]^{-1} \mathbf{r}_{0}
$$




\section{References}

[1] M. Gustavsson, J. J. Wikner, and N. Tan, CMOS Data Converters for Communications. Springer, 2000.

[2] J. Singh, S. Ponnuru, and U. Madhow, "Multi-gigabit communication: the ADC bottleneck," in Proc. IEEE Int. Conf. Ultra-Wideband, Vancouver, BC, Sep. 2009, pp. 22-27.

[3] W. C. Black and D. A. Hodges, "Time interleaved converter arrays," IEEE J. Solid-State Circuits, vol. 15, no. 6, pp. 1022-1029, Dec. 1980.

[4] A. Petraglia and S. Mitra, "Analysis of mismatch effects among A/D converters in a time-interleaved waveform digitizer," IEEE Trans. Instrum. Meas., vol. 40, no. 5, pp. 831-835, Oct. 1991.

[5] P. Harikumar, "Building Blocks for Low-Voltage Analog-to-Digital Interfaces," Licentiate thesis, Linköping University, Sweden, Nov. 2014.

[6] H. Masuda, S. Ohkawa, A. Kurokawa, and M. Aoki, "Challenge: variability characterization and modeling for 65- to 90-nm processes," in Proc. IEEE Custom Integrated Circuits Conf., San Jose, CA, USA, Sep. 2005, pp. 593-599.

[7] S. Nassif, K. Bernstein, D. J. Frank, A. Gattiker, W. Haensch, B. L. Ji, E. Nowak, D. Pearson, and N. J. Rohrer, "High performance CMOS variability in the $65 \mathrm{~nm}$ regime and beyond," in Proc. IEEE Int. Electron. Devices Meeting, Washington, DC, USA, Dec. 2007, pp. 569-571.

[8] S. Bhunia, S. Mukhopadhyay, and K. Roy, "Process variations and processtolerant design," in Proc. IEEE Int. Conf. VLSI Design, Bangalore, India, Jan. 2007, pp. 699-704.

[9] N. Kurosawa, H. Kobayashi, and K. Kobayashi, "Channel linearity mismatch effects in time-interleaved ADC systems," in Proc. IEEE Int. Symp. Circuits Syst., vol. 1, Sydney, Australia, May 2001, pp. 420-423.

[10] N. Kurosawa, H. Kobayashi, K. Maruyama, H. Sugawara, and K. Kobayashi, "Explicit analysis of channel mismatch effects in time-interleaved ADC systems," IEEE Trans. Circuits Syst. I, vol. 48, no. 3, pp. 261-271, Mar. 2001. 
[11] C. Vogel, "Modeling, Identification, and Compensation of Channel Mismatch Errors in Time-Interleaved Analog-to-Digital Converters," Ph.D. dissertation, Graz University of Technology, Austria, Jul. 2005.

[12] C. Vogel and G. Kubin, "Modeling of time-interleaved ADCs with nonlinear hybrid filter banks," AEU-Int. J. Electron. Commun., vol. 59, no. 5, pp. 288296, Jul. 2005.

[13] Y. Wang, H. Johansson, and H. Xu, "Adaptive background estimation for static nonlinearity mismatches in two-channel TIADCs," IEEE Trans. Circuits Syst. $I I$, vol. 62, no. 3, pp. 226-230, Mar. 2015.

[14] Y. Wang, H. Johansson, H. Xu, and Z. Sun, "Joint blind calibration for mixed mismatches in two-channel time-interleaved ADCs," IEEE Trans. Circuits Syst. I, accepted.

[15] C. Vogel and H. Johansson, "Time-interleaved analog-to-digital converters: status and future directions," in Proc. IEEE Int. Symp. Circuits Syst., Kos, Greece, May 2006, pp. 3386-3389.

[16] J. Elbornsson, F. Gustafsson, and J.-E. Eklund, "Blind adaptive equalization of mismatch errors in a time-interleaved A/D converter system," IEEE Trans. Circuits Syst. I, vol. 51, no. 1, pp. 151-158, Jan. 2004.

[17] C.-C. Huang, C.-Y. Wang, and J.-T. Wu, "A CMOS 6-bit 16-GS/s timeinterleaved ADC using digital background calibration techniques," IEEE J. Solid-State Circuits, vol. 46, no. 4, pp. 848-858, Apr. 2011.

[18] S. Tertinek and C. Vogel, "Reconstruction of nonuniformly sampled bandlimited signals using a differentiator-multiplier cascade," IEEE Trans. Circuits Syst. I, vol. 55, no. 8, pp. 2273-2286, Sep. 2008.

[19] L. Zhao, X. Hu, C. Feng, S. Tang, S. Liu, and Q. An, "A 1.6-Gsps highresolution waveform digitizer based on a time-interleaved technique," IEEE Trans. Nucl. Sci., vol. 60, no. 3, pp. 2180-2187, Jun. 2013.

[20] H. Johansson and P. Löwenborg, "A least-squares filter design technique for the compensation of frequency response mismatch errors in time-interleaved A/D converters," IEEE Trans. Circuits Syst. II, vol. 55, no. 11, pp. 1154-1158, Nov. 2008 .

[21] S. Saleem and C. Vogel, "Adaptive blind background calibration of polynomialrepresented frequency response mismatches in a two-channel time-interleaved ADC," IEEE Trans. Circuits Syst. I, vol. 58, no. 6, pp. 1300-1310, Dec. 2011.

[22] S. Lee, A. P. Chandrakasan, and H.-S. Lee, "A 1GS/s 10b $18.9 \mathrm{~mW}$ timeinterleaved SAR ADC with background timing-skew calibration," in IEEE Int. Solid-State Circuits Conf. Dig. Tech. Papers, San Francisco, CA, USA, Feb. 2014, pp. 384-385. 
[23] E. Janssen, K. Doris, A. Zanikopoulos, A. Murroni, G. van der Weide, Y. Lin, L. Alvado, F. Darthenay, and Y. Fregeais, "An 11b 3.6GS/s time-interleaved SAR ADC in 65nm CMOS," in IEEE Int. Solid-State Circuits Conf. Dig. Tech. Papers, San Francisco, CA, USA, Feb. 2013, pp. 464-465.

[24] N. Le Dortz, J.-P. Blanc, T. Simon, S. Verhaeren, E. Rouat, P. Urard, S. Le Tual, D. Goguet, C. Lelandais-Perrault, and P. Benabes, "A 1.62GS/s time-interleaved SAR ADC with digital background mismatch calibration achieving interleaving spurs below 70dBFS," in IEEE Int. Solid-State Cicuits Conf. Dig. Tech. Papers, San Francisco, CA, USA, Feb. 2014, pp. 386-388.

[25] M. Mishali and Y. Eldar, "Sub-Nyquist sampling," IEEE Signal Process. Mag., vol. 28 , no. 6 , pp. $98-124$, Nov. 2011.

[26] K. M. Tsui and S. C. Chan, "A novel iterative structure for online calibration of $M$-channel time-interleaved ADCs," IEEE Trans. Instrum. Meas., vol. 63, no. 2, pp. 312-325, Feb. 2014.

[27] H. Nyquist, "Certain topics in telegraph transmission theory," Trans. Amer. Inst. Elect. Eng., vol. 47, no. 2, pp. 617-644, Apr. 1928.

[28] V. A. Kotelnikov, "On the transmission capacity of "ether" and wire in electrocommunications," Izd. Red. Upr. Svyazi RKKA (Moscow), 1933.

[29] C. E. Shannon, "Communication in the presence of noise," Proc. IRE, vol. 37, pp. 10-21, 1949.

[30] H. Johansson, "Sampling and quantization," in Academic Press Library in Signal Processing: Signal Processing Theory and Machine Learning, ser. Academic Press Library in Signal Processing, P. S. Diniz, J. A. Suykens, R. Chellappa, and S. Theodoridis, Eds. Elsevier, 2014, vol. 1, ch. 5, pp. 169-244.

[31] T. Saramäki, "Finite impulse response filter design," in Handbook for Digital Signal Processing, S. Mitra and J. Kaiser, Eds. Wiley, Newyork, 1993, ch. 4, pp. 155-277.

[32] P. P. Vaidyanathan, Multirate Systems and Filter Banks. Prentice-Hall, Englewood Cliffs, NJ, USA, 1993.

[33] f. harris, Multirate Signal Processing for Communication Systems. Prentice Hall, 2004.

[34] P. P. Vaidyanathan, "Multirate digital filters, filter banks, polyphase networks, and applications: a tutorial," Proc. IEEE, vol. 78, no. 1, pp. 56-93, Jan. 1990.

[35] T. Saramäki and R. Bregovic, "Multirate systems and filter banks," in Multirate Systems: Design and Applications, G. Jovanovic-Dolecek, Ed. Idea Group Publishing, Hershey PA, 2002, ch. 2, pp. 27-85.

[36] H. S. Malvar, "Extended lapped transforms: properties, applications, and fast algorithms," IEEE Trans. Signal Process., vol. 40, no. 11, pp. 2703-2714, Nov. 1992. 
[37] A. Viholainen, J. Alhava, and M. Renfors, "Efficient implementation of complex modulated filter banks using cosine and sine modulated filter banks," EURASIP J. Advances Signal Process., vol. 2006, pp. 1-10, Apr. 2006.

[38] F. R. Gantmacher, Matrix Theory. American Mathematical Society, 2000, vol. 2.

[39] D. Kalman, "The generalized Vandermonde matrix," Math. Mag., vol. 57, no. 1, pp. 15-21, Jan. 1984.

[40] M. El-Chammas and B. Murmann, "A 12-GS/s 81-mW 5-bit time-interleaved flash ADC with background timing skew calibration," IEEE J. Solid-State Circuits, vol. 46, no. 4, pp. 838-847, Apr. 2011.

[41] T.-H. Tsai, P. J. Hurst, and S. H. Lewis, "Bandwidth mismatch and its correction in time-interleaved analog-to-digital converters," IEEE Trans. Circuits Syst. II, vol. 53, no. 10, pp. 1133-1137, Oct. 2006.

[42] J. Elbornsson, F. Gustafsson, and J. E. Eklund, "Blind equalization of time errors in a time-interleaved ADC system," IEEE Trans. Signal Process., vol. 53, no. 4, pp. 1413-1424, Apr. 2005.

[43] V. Divi and G. Wornell, "Scalable blind calibration of timing skew in highresolution time-interleaved ADCs," in Proc. IEEE Int. Symp. Circuits Syst., Kos, Greece, May 2006, pp. 3390-3393.

[44] S. Huang and B. C. Levy, "Adaptive blind calibration of timing offset and gain mismatch for two-channel time-interleaved ADCs," IEEE Trans. Circuits Syst. I, vol. 53, no. 6, pp. 1278-1288, Jun. 2006.

[45] S. Huang and B. C. Levy, "Blind calibration of timing offsets for four-channel time-interleaved ADCs," IEEE Trans. Circuits Syst. I, vol. 54, no. 4, pp. 863876, Apr. 2007.

[46] A. Haftbaradaran and K. W. Martin, "A background sample-time error calibration technique using random data for wide-band high-resolution timeinterleaved ADCs," IEEE Trans. Circuits Syst. II, vol. 55, no. 3, pp. 234-238, Mar. 2008.

[47] M. El-Chammas and B. Murmann, "General analysis on the impact of phaseskew in time-interleaved ADCs," IEEE Trans. Circuits Syst. I, vol. 56, no. 5, pp. 902-910, May 2009.

[48] M. Seo, M. J. W. Rodwell, and U. Madhow, "Comprehensive digital correction of mismatch errors for a 400-M samples/s 80-dB SFDR time-interleaved analogto-digital converter," IEEE Trans. Microw. Theory Tech., vol. 53, no. 3, pp. 1072-1082, Mar. 2005.

[49] S. Mendel and C. Vogel, "A compensation method for magnitude response mismatches in two-channel time-interleaved analog-to-digital converters," in Proc. IEEE Int. Conf. Electron. Circuits Syst., Nice, France, Dec. 2006, pp. $712-715$. 
[50] S. Mendel and C. Vogel, "On the compensation of magnitude response mismatches in $M$-channel time-interleaved ADCs," in Proc. IEEE Int. Symp. Circuits Syst., New Orleans, LA, USA, May 2007, pp. 3375-3378.

[51] M. Seo, M. Rodwell, and U. Madhow, "Generalized blind mismatch correction for two-channel time-interleaved A-to-D converters," in Proc. IEEE Int. Conf. Acoust., Speech, Signal Process., vol. 3, Honolulu, HI, USA, Apr. 2007, pp. $1505-1508$.

[52] M. Seo and M. Rodwell, "Generalized blind mismatch correction for a twochannel time-interleaved ADC: Analytic approach," in Proc. IEEE Int. Symp. Circuits Syst., New Orleans, LA, USA, May 2007, pp. 109-112.

[53] P. Satarzadeh, B. C. Levy, and P. J. Hurst, "Bandwidth mismatch correction for a two-channel time-interleaved A/D converter," in Proc. IEEE Int. Symp. Circuits Syst., New Orleans, LA, USA, May 2007, pp. 1705-1708.

[54] H. Johansson, "A polynomial-based time-varying filter structure for the compensation of frequency-response mismatch errors in time-interleaved ADCs," IEEE J. Sel. Topics Signal Process., vol. 3, no. 3, pp. 384-396, Jun. 2009.

[55] Y. C. Lim, Y.-X. Zou, J. W. Lee, and S.-C. Chan, "Time-interleaved analog-todigital-converter compensation using multichannel filters," IEEE Trans. Circuits Syst. I, vol. 56, no. 10, pp. 2234-2247, Oct. 2009.

[56] C. Vogel and S. Mendel, "A flexible and scalable structure to compensate frequency response mismatches in time-interleaved ADCs," IEEE Trans. Circuits Syst. I, vol. 56, no. 11, pp. 2463-2475, Nov. 2009.

[57] H. Johansson and P. Löwenborg, "Reconstruction of nonuniformly sampled bandlimited signals by means of time-varying discrete-time FIR filters," EURASIP J. Advances Signal Process., vol. 2006, pp. 1-18, Jan. 2006.

[58] Y. C. Eldar and A. V. Oppenheim, "Filterbank reconstruction of bandlimited signals from nonuniform and generalized samples," IEEE Trans. Signal Process., vol. 48, no. 10, pp. 2864-2875, Oct. 2000.

[59] H. Johansson, P. Löwenborg, and K. Vengattaramane, "Least-squares and minimax design of polynomial impulse response FIR filters for reconstruction of two-periodic nonuniformly sampled signals," IEEE Trans. Circuits Syst. I, vol. 54, no. 4, pp. 877-888, Apr. 2007.

[60] A. V. Oppenheim and R. W. Schafer, Discrete-Time Signal Processing. Prentice-Hall Signal Processing Series, 1989.

[61] H. Johansson, Discrete-Time Systems. LiuTryck, 2009.

[62] "IEEE standard for terminology and test methods for analog-to-digital converters," IEEE Std 1241-2000, 2001.

[63] J. Selva, "Functionally weighted lagrange interpolation of band-limited signals from nonuniform samples," IEEE Trans. Signal Process., vol. 57, no. 1, pp. 168-181, Jan. 2009. 
[64] L. Wanhammar, DSP Integrated Circuits. Academic Press, 1999, ch. 11, pp. $461-530$.

[65] O. Gustafsson, "Lower bounds for constant multiplication problems," IEEE Trans. Circuits Syst. II, vol. 54, no. 11, pp. 974-978, Nov. 2007.

[66] N. P. Murphy, A. Krukowski, and I. Kale, "Implementation of wideband integer and fractional delay element," Electron. Lett., vol. 30, no. 20, pp. 1658-1659, Sep. 1994.

[67] G. Jovanovic-Dolecek and J. Diaz-Carmona, "One structure for widebandwidth and high-resolution fractional delay filter," in Proc. IEEE Int. Conf. Electron. Circuits Syst., vol. 3, Dubrovnik, Croatia, Sep. 2002, pp. 899-902.

[68] E. Hermanowicz, "On designing a wideband fractional delay filter using the Farrow approach," in Proc. XII European Signal Process. Conf., Vienna, Austria, Sep. 2004, pp. 961-964.

[69] E. Hermanowicz and H. Johansson, "On designing minimax adjustable wideband fractional delay FIR filters using two-rate approach," in Proc. European Conf. Circuit Theory Design, vol. 3, Cork, Ireland, Sep. 2005, pp. 437-440.

[70] H. Johansson, O. Gustafsson, J. Johansson, and L. Wanhammar, "Adjustable fractional-delay FIR filters using the Farrow structure and multirate techniques," in Proc. IEEE Asia Pacific Conf. Circuits Syst., Singapore, Dec. 2006, pp. $1055-1058$.

[71] J. Diaz-Carmona, G. Jovanovic-Dolecek, and A. Ramirez-Agundis, "Frequency-based optimization design for fractional delay FIR filters with software-defined radio applications," Int. J. Digital Multimedia Broadcast., vol. 2010, pp. 1-6, Jun. 2010.

[72] Z. U. Sheikh and H. Johansson, "A class of wide-band linear-phase FIR differentiators using a two-rate approach and the frequency-response masking technique," IEEE Trans. Circuits Syst. I, vol. 58, no. 8, pp. 1827-1839, Aug. 2011.

[73] Z. U. Sheikh and H. Johansson, "Efficient wide-band FIR LTI systems derived via multi-rate techniques and sparse bandpass filters," IEEE Trans. Signal Process., vol. 60, no. 7, pp. 3859-3863, Jul. 2012.

[74] H. Johansson and E. Hermanowicz, "Two-rate based low-complexity variable fractional-delay FIR filter structures," IEEE Trans. Circuits Syst. I, vol. 60, no. 1, pp. 136-149, Jan. 2013.

[75] H. Johansson and O. Gustafsson, "Two-rate based structure for computationally efficient wide-band FIR systems," in Digital Filters and Signal Processing, F. P. G. Márquez and N. Zaman, Eds. InTech, 2013, ch. 8, pp. 189-212.

[76] f. harris, X. Chen, E. Venosa, and F. A. N. Palmieri, "Two channel TI-ADC for communication signals," in Proc. IEEE Int. Workshop Signal Process. Advances Wireless Commun., San Francisco, CA, USA, Jun. 2011, pp. 576-580. 
[77] S. M. Jamal, D. Fu, M. P. Singh, P. J. Hurst, and S. H. Lewis, "Calibration of sample-time error in a two-channel time-interleaved analog-to-digital converter," IEEE Trans. Circuits Syst. I, vol. 51, no. 1, pp. 130-139, Jan. 2004.

[78] [Online]. Available: http://spdevices.com/index.php/products2

[79] S. Tertinek and C. Vogel, "Reconstruction of two-periodic nonuniformly sampled band-limited signals using a discrete-time differentiator and a time-varying multiplier," IEEE Trans. Circuits Syst. II, vol. 54, no. 7, pp. 616-620, Jul. 2007.

[80] H. Johansson, P. Löwenborg, and K. Vengattaramane, "Reconstruction of $M$-periodic nonuniformly sampled signals using multivariate impulse response time-varying FIR filters," in Proc. XIV European Signal Process. Conf., Florence, Italy, Sep. 2006, pp. 1-4.

[81] Y.-P. Lin and P. Vaidyanathan, "Periodically nonuniform sampling of bandpass signals," IEEE Trans. Circuits Syst. II, vol. 45, no. 3, pp. 340-351, Mar. 1998.

[82] R. Venkataramani and Y. Bresler, "Optimal sub-Nyquist nonuniform sampling and reconstruction for multiband signals," IEEE Trans. Signal Process., vol. 49, no. 10, pp. 2301-2313, Oct. 2001.

[83] N. D. Blakeley, P. J. Bones, R. P. Millane, and P. Renaud, "Efficient frequencydomain sample selection for recovering limited-support images," J. Optical Soc. America. Optics Image Sci. Vision, vol. 20, no. 1, pp. 67-77, Jan. 2003.

[84] A. Owrang, M. Viberg, M. Nosratinia, and M. Rashidi, "A new method to compute optimal periodic sampling patterns," in Proc. IEEE Digital Signal Process. Workshop, Sedona, AZ, USA, Jan. 2011, pp. 259-264.

[85] M. Renfors and Y. Neuvo, "The maximum sampling rate of digital filters under hardware speed constraints," IEEE Trans. Circuits Syst., vol. 28, no. 3, pp. 196-202, Mar. 1981.

[86] H. Johansson and A. Eghbali, "FIR filter with variable fractional delay and phase shift: Efficient realization and design using reweighted $\ell_{1}$-norm minimization," in Proc. IEEE Int. Symp. Circuits Syst., Beijing, China, May 2013, pp. 81-84.

[87] H. Johansson and P. Löwenborg, "Reconstruction of nonuniformly sampled bandlimited signals by means of digital fractional delay filters," IEEE Trans. Signal Process., vol. 50, no. 11, pp. 2757-2767, Nov. 2002.

[88] A. K. M. Pillai and H. Johansson, "Efficient signal reconstruction scheme for time-interleaved ADCs," in Proc. IEEE Int. New Circuits Syst. Conf., Montreal, Canada, Jun. 2012, pp. 357-360.

[89] A. K. M. Pillai and H. Johansson, "Time-skew error correction in two-channel time-interleaved ADCs based on a two-rate approach and polynomial impulse responses," in Proc. IEEE Int. Midwest Symp. Circuits Syst., Boise, ID, USA, Aug. 2012, pp. 1136-1139. 
[90] A. K. M. Pillai and H. Johansson, "Efficient reconfigurable scheme for the recovery of sub-Nyquist sampled sparse multi-band signals," in Proc. IEEE Global Conf. Signal Information Process., Austin, TX, USA, Dec. 2013, pp. 1294-1297.

[91] M. Bellanger, G. Bonnerot, and M. Coudreuse, "Digital filtering by polyphase network:application to sample-rate alteration and filter banks," IEEE Trans. Acoust., Speech, Signal Process., vol. 24, no. 2, pp. 109-114, Apr. 1976.

[92] H. Johansson and A. Eghbali, "Two polynomial FIR filter structures with variable fractional delay and phase shift," IEEE Trans. Circuits Syst. I, vol. 61, no. 5, pp. 1355-1365, May 2014. 


\section{Part II}

\section{Efficient Reconstruction Schemes for TI-ADCs}





\section{Papers}

The articles associated with this thesis have been removed for copyright reasons. For more details about these see:

http://urn.kb.se/resolve?urn=urn:nbn:se:liu:diva-117826 